Universidade de São Paulo
Instituto de Física de São Carlos

Prof. Dr. Luiz Vitor de Souza Filho

Astrofísica de Partículas: detecção, propagação e geração de partículas de altíssima energia 

Prof. Dr. Luiz Vitor de Souza Filho

\section{Astrofísica de Partículas: detecção, propagação e geração de partículas de altíssima energia}

Tese apresentada no concurso de títulos e provas visando à obtenção do título de livredocente no Departamento de Física e Ciência Interdisciplinar.

Área de Conhecimento: Astrofísica de Partículas

Versão Original

São Carlos 


\begin{abstract}
AUTORIZO A REPRODUÇÃO TOTAL OU PARCIAL DESTE TRABALHO, POR QUALQUER MEIO CONVENCIONAL OU ELETRÔNICO, PARA FINS DE ESTUDO E PESQUISA, DESDE QUE CITADA A FONTE.
\end{abstract}

Ficha catalográfica elaborada pelo Serviço de Biblioteca e Informação do IFSC

Souza Filho, Luiz Vitor de

Astrofísica de partículas: detecção, propagação e geração de partículas de altíssima energia/ Luiz Vitor de Souza Filho. -- São Carlos: IFSC, 2015.

$76 \mathrm{p}$.

Tese (Livre Docência) - Programa de Pós-Graduação em Astrofísica de Partículas) - Instituto de Física de São Carlos, Universidade de São Paulo, 2015.

1. Astrofísica de partículas. 2. Observatório Pierre Auger. 3. Propagação extragalática. 4. Radio galáxias. I. Título. 
À minha irmã. 



\section{AGRADECIMENTOS}

Esta tese é dedicada à minha irmã, constante inspiração e companhia.

Invariavelmente, o trabalho de pesquisa é um exercício de solidão. Mesmo se realizado em colaboração, o surgimento de uma ideia é sempre um acontecimento mental individual e insulado pelas divagações do pesquisador. O estágio de desenvolvimento das ciências naturais implica que a maior parte das hipóteses concebidas levam muito tempo, às vezes anos, de trabalho solitário para serem concluídas. A grande maioria delas serão falsificadas o que aumenta a sensação de trabalho vão. As raras ideias que se mostrarem verdadeiras ganham o direito se serem publicadas ou se concretizarão em um instrumento ou um gráfico.

Apesar disso, me sinto muito feliz por perceber que tenho muitas pessoas pra agradecer nesta tese. Muitas pessoas fizeram parte de uma ou outra maneira nesta trajetória que tem uma etapa cumprida com a escrita desta tese.

Minha família: pai, mãe, Thana e Bruno são coautores desta tese por letras, equações, gráficos e instrumentos escritos no meu coração. Muito obrigado por tudo que vivemos nestes anos. À minha mulher, tenho que agradecer imensamente pelo amor incondicional e pela construção da nossa família em concomitância com esta tese. Danke Gabi. Para agradecer e registrar a importância do meu filho durante os anos que este trabalho se desenvolveu me faltam engenho e arte, por isso cito. O Marc "(...) é minha explicação, meu verso melhor ou único, meu tudo enchendo meu nada." *.

Carlos José Todero Peixoto, Daniel Supanitsky, Peter Biermann, Raul Prado Ribeiro e Rita de Cássia do Anjos são coautores da física escrita nesta tese. Agradeço muito pela oportunidade de trabalhar com vocês. Tenho sido um privilegiado de participar da Colaboração Pierre Auger na qual tenho aprendido muito nas discussões de trabalho, sobretudo com José Bellido e Michael Unger.

Nestes anos, construímos um grupo de pesquisa em Astrofísica de Partículas no IFSC e esta tese reflete este processo. Além dos nomes já citados acima, agradeço aos alunos que trabalharam comigo nestes anos: Gustavo Salinas de Souza, Hendrik Marques Soares, Jéssica Arab Marcomini, Jessica Dipold, Mateus Arantes Fandino, Rodrigo Guedes Lang e Victor Barbosa Martins.

* A mesa, Drummond 
Muitos amigos participaram desta tese e agradeço a todos pela companhia pessoal e/ou acadêmica e/ou científica: Cibelle Celestino, Edivaldo Santos, Leonardo Maia, Marcelo Barros, Marcelo Leigui, Mateus Gerolamo, Silvio Levcovitz, Simone Yamauti e Vivian da Cunha. Obrigado pelo tempo que dividimos enquanto esta tese era gerada. Ao Thiago Mosqueiro agradeço pela elaboração do estilo Latex IFSC com o qual esta tese foi escrita.

Agradeço também a todos do IFSC sem os quais meu trabalho teria sido muito mais difícil ou praticamente impossível: Claudia Tofaneli, Cristina da Silva, Sonia dos Santos, Thais Pinto, a todos da biblioteca, a todos da assessoria de comunicação, a todos da gráfica, a todos da assistência técnica administrativa, a todos da assistência técnica acadêmica, a todos da assistência técnica financeira e a todos da seção técnica de informática. Agradeço institucionalmente ao IFSC pelo apoio ao meu trabalho através da citação dos seus diretores desde a minha contratação: Glaucius Oliva, Antonio Carlos Hernandes e Tito José Bonagamba.

Agradeço por último ao povo brasileiro pelo financiamento do meu trabalho através da USP, da CAPES, do CNPq e da FAPESP. A ciência e a Universidade são esforços coletivos da sociedade. 
"E, sendo assim, o que o leitor fará é também sua criação, um começo absoluto, o qual só pode ser realizado por meio da liberdade. O escritor apela, portanto, para a liberdade do leitor, para a sua cooperação na criação da obra."

\section{Thana Mara de Souza}

"Quem viu Garrincha atuar não pode levar a sério teorias científicas que reveem a parábola inevitável de uma bola e asseguram a vitória - que não acontece."

Carlos Drummond de Andrade 



\section{RESUMO}

DE SOUZA FILHO, L. V. Astrofísica de Partículas: detecção, propagação e geração de partículas de altíssima energia. 2015. 104p. Tese de Livre-docência - Instituto de Física de São Carlos, Universidade de São Paulo, São Carlos, 2015.

Nesta tese, discutiremos os avanços em Astrofísica de Partículas da última década com ênfase na nossa contribuição para a detecção e análise de dados, propagação de partículas no meio intergalático e aceleração de partículas em radio galáxias. No capítulo 2, explicaremos o funcionamento e análise de dados do Observatório Pierre Auger e discutiremos em mais detalhes nossa participação nos esforços de determinação do tipo da partícula primária. Revisaremos os principais resultados publicados pela Colaboração Auger a respeito do espectro de energia, da composição de massa e da direção de chegada dos eventos. No capítulo 3, revisaremos os fenômenos mais importantes que interferem na propagação de raios cósmicos de altas energias com foco na produção de raios gama com energia entre $\mathrm{GeV}$ e TeV. Mostraremos um estudo de revisão das radiações de fundo e da contribuição de cada componente na modulação do espectro de energia detectado na Terra. Discutiremos em detalhes um modelo por nós proposto de cálculo no limite da luminosidade em raios cósmicos de fontes pontuais utilizando medidas do limite da integral do fluxo de raios gamas de GeV-TeV. Ilustraremos a utilização do modelo calculando o limite na luminosidade de raios cósmicos de 30 fontes estudadas pelos Observatório Fermi-LAT, H.E.S.S., MAGIC e VERITAS. No capítulo 4, descrevemos um modelo de aceleração de partículas elaborado originariamente em 1993 por Stanev, Biermann e Gaisser. Neste modelo, apenas a nossa galáxia e a radio galáxia Centaurus A são utilizadas para explicar o fluxo de raios cósmicos com energia entre $10^{10}$ e $10^{20} \mathrm{eV}$. Trabalhamos com este modelo original e ajustamos suas variáveis livres à dados recentes. Impusemos limites e fixamos alguns dos graus de liberdade do modelo e exploramos suas qualidades na descrição dos resultados experimentais. No capítulo 5, discutimos algumas perspectivas futuras do nosso trabalho incluindo as propostas experimentais de construção do AugerPrime e CTA.

PAlavras-Chave: Astrofísica de partículas, Observatório Pierre Auger, Propagação extragalática, Rádio galáxias. 



\section{ABSTRACT}

DE SOUZA FILHO, L. V. Astroparticle Physics: detection, propagation and acceleration of the highest energectic particles.. 2015. 104p. Tese de Livre-docência - Instituto de Física de São Carlos, Universidade de São Paulo, São Carlos, 2015.

In this thesis the development of Astroparticle Phyisics in the last decade is discussed with emphasis to the contribution we gave to instrumentation and data analysis, to model the propagation of particles in the intergalactic medium and to theories in particle acceleration in radio galaxies. In chapter 2 we explain the Pierre Auger Observatory and the data analysis used to extract the main scientific results published so far concerning the energy spectrum, the mass composition and the arrival direction of events. We focus on our contribution to the mass composition determination. In chapter 3 we review the main phenomena that modulates the original flux of particles until its arrival on Earth. We discuss the detalhes of a method proposed by us which allows the determination of an upper limit on the cosmic ray luminosity of point sources based on measurements done by gamma-ray observatories of the upper limit of the integral of the flux of gama rays with energy between $\mathrm{GeV}$ and $\mathrm{TeV}$. We illustrate the method by calculating the upper limit on the cosmic-ray luminosity of 30 sources studied by FERMI-LAT, H.E.S.S., MAGIC na VERITAS. In chapter 4 we describe the original model proposed in 1993 by Stanev, Biermann e Gaisser in which our Galaxy and the radio galaxy Centaurus $A$ are able to produce the cosmic ray flux observed on Earth in the energy range from $10^{10}$ to $10^{20} \mathrm{eV}$. We managed to impose limits and fix several parameters of the original model by fiting it to the most updated data. In chapter 5 we discuss some experimental future perspectives of our work related to the construction of AugerPrime and CTA.

KeywORDS: Astroparticle physics, Pierre Auger Observatory, Extragalactic Propagation, Radio galaxies. 



\section{LISTA DE FIGURAS}

2.1 Mapa do Observatório Auger. Os pontos mostram os detectores de superfície. As linhas azuis e vermelhas representam o campo de visão de cada telescópio de fluorescência. Dois pontos vermelhos no meio do arranjo de detectores de superfície: XLF (eXtreme Laser Facility) e CLF (Central Laser Facility), mostram a posição de instalação de monitoramento das condições atmosféricas. O ponto denominado Central Campus mostra a posição do central de operações do Observatório na cidade de Malargüe. Fonte: Figura produzida pela Colaboração Pierre Auger disponível apenas para os membros. p. 34

2.2 Esquema da detecção de um chuveiro atmosférico pelo Observatório Pierre Auger. Os círculos representam todos os detectores de superfície em operação. Os círculos maiores no dentro da imagem mostram os detectores que mediram a passagem de partículas do chuveiro. As figuras sextavadas na borda do conjunto de detectores de superfície representam os telescópios de fluorescência e seus campos de visão. As linhas saindo de cada telescópio representam o centro de cada pixel disparado pelo chuveiro. As linhas verticais mostram a reconstrução da direção de chegada deste evento. Quatro reconstruções da direção são mostradas, cada uma usando os dados de um telescópio de fluorescência. Fonte: Figura produzida pela Colaboração Pierre Auger disponível apenas para os membros. . . . . . . . . . . . . . . .

2.3 Exemplo de um evento medido pelo telescópio de fluorescência. Esquerda: Luz detectada pelo telescópio em função do tempo. São mostradas em separado as contribuições estimadas devido efeitos de espalhamentos simples e múltiplos (Mie e Rayleigh) e contaminação por emissão Čerenkov. Direita: Perfil de energia depositada em função da profundidade atmosférica. A linha preta mostra um ajuste de uma função Gaisser-Hillas (1) aos dados medidos. Fonte: Figura produzida pela Colaboração Pierre Auger disponível apenas para os membros. 
2.4 Esquerda: Exemplo da densidade de partículas em função da distância em relação ao eixo do chuveiro. Os pontos pretos mostram os dados medidos. Os quadrados pretos mostram as estações com sinal acima do nível de saturação cujo método de reconstrução foi capaz de estimar o sinal verdadeiro. Os quadrados abertos mostram as estações com sinal acima do nível de saturação cujo método de reconstrução não foi capaz de estimar o sinal verdadeiro. Os triângulos mostram as estações na redondeza que não foram disparadas pelo chuveiro. O círculo aberto mostra uma estação classificada como não pertencente ao evento mesmo contendo sinal diferente de zero por uma verificação de coerência de disparo temporal. A linha preta mostra o ajuste de uma função ao dados medidos. A área hachurada mostra a incerteza de um sigma do ajuste da função. O ponto vermelho mostra o valor do sinal a 1000 metros do eixo do chuveiro. Direita: Calibração da energia usando eventos medidos simultaneamente pelos detectores de superfície e pelos telescópios de fluorescência através da relação entre $S_{38}$ e $E_{F D}$. Os pontos pretos são os dados medidos e a linha vermelha o ajuste de uma reta aos dados. Fonte: Figura produzida pela Colaboração Pierre Auger disponível apenas para os membros. . . . . . . . . . . . . . . . .

2.5 Evolução da média da distribuição de $X_{\max }$ em função da massa da partícula primária com várias energias (cores). A figura mostra resultados de simulação de Monte Carlo de chuveiros atmosféricos utilizando o programa CORSIKA (2) como modelo de interação hadrônica Sibyll 2.1 (3). Fonte:

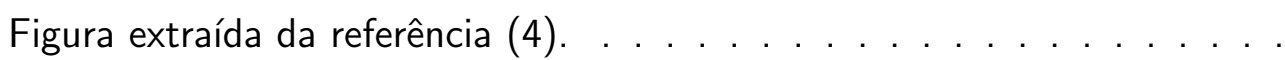

2.6 Distribuição da diferença da reconstrução do valor de $X_{\max }$ para eventos medidos por dois telescópios de fluorescência. Os pontos pretos mostram os dados e a linha contínua o calculo utilizando simulação de Monte Carlo (MC) dos detectores. Fonte: Figura produzida pela Colaboração Pierre Auger disponível apenas para os membros. . . . . . . . . . . . . . .

2.7 Espectro de energia medido pelo Observatório Pierre Auger (5). Fonte: Figura produzida pela Colaboração Pierre Auger disponível apenas para os membros. . . . . . . . . . . . . . . . . 
2.8 Mapa da direção de chegada dos eventos (pontos pretos) medidos pelo Observatório Pierre Auger com energia superior a $58 \mathrm{EeV}$ em comparação com a posição de Active Galactic Nucleis (AGNs) no catálogo Swift-BAT (círculos vermelhos - janela de $1^{\circ}$ ) com distância máxima de $80 \mathrm{Mpc}$. O mapa foi feito em coordenadas galáticas e projeção Aitoff-Hammer. Fonte: Figura produzida pela Colaboração Pierre Auger disponível apenas para os membros.

2.9 Mapa da direção de chegada dos eventos (pontos pretos) medidos pelo Observatório Pierre Auger com energia superior a $58 \mathrm{EeV}$ em comparação com a posição de AGNs no catálogo Swift-BAT (círculos vermelhos - janela de $18^{\circ}$ ) com distância máxima de $130 \mathrm{Mpc}$ e luminosidade máxima de $10^{44} \mathrm{erg}$ $\mathrm{s}^{-1}$. O mapa foi feito em coordenadas galáticas e projeção Aitoff-Hammer. Fonte: Figura produzida pela Colaboração Pierre Auger disponível apenas para os membros.

2.10 Distribuição do número de eventos em função da distância angular de Centaurus $A$ em comparação com a predição de isotropia. Dados medidos pelo Observatório Pierre Auger (6). Fonte: Figura produzida pela Colaboração Pierre Auger disponível apenas para os membros.

2.11 Ilustração do enviesamento da distribuição de $X_{\max }$ devido à seletividade do campo de visão dos telescópios de fluorescência. O painel da esquerda mostra o campo de visão dos telescópios de fluorescência (linhas tracejadas) sendo cortado por geometrias representativas de chuveiros ( $A, B$ e $C$ ). $O$ painel da direita mostra as distribuições de $X_{\max }$ correspondentes às geometrias. Fonte: Figura produzida pela Colaboração Pierre Auger disponível apenas para os membros.

2.12 Evolução dos extremos do campo de visão (Xlow e Xup) em função de $X_{\max }$. Fonte: Figura produzida pela Colaboração Pierre Auger disponível apenas para os membros.

2.13 Evolução do logaritmo da massa da partícula primária $(\ln (A))$ em função da energia. Comparação entre as técnicas de remoção de vieses e distorções experimentais. Medidas realizadas pelo Observatório Pierre Auger (7). A legenda "análise padrão" refere-se aos cortes fiduciais. Fonte: Figura extraída da referência $(8) \ldots \ldots \ldots \ldots \ldots \ldots \ldots \ldots . \ldots \ldots \ldots$ 
2.14 Distribuições de $X_{\max }$ em vários intervalos de energia. Fonte: Figura produzida pela Colaboração Pierre Auger disponível apenas para os membros.

2.15 Evolução dos momentos centrais (média e RMS) das distribuições de $X_{\max }$ em função da energia. As linhas mostram predição de simulações para chuveiros iniciados por prótons e núcleos de ferro. Medidas realizadas pelo Observatório Pierre Auger (7). Fonte: Figura produzida pela Colaboração Pierre Auger disponível apenas para os membros. . . . . . . . . . . . . . .

2.16 Evolução do logaritmo da massa da partícula primária $(\ln (A))$ em função da energia. Medidas realizadas pelo Observatório Pierre Auger (7). Fonte: Figura produzida pela Colaboração Pierre Auger disponível apenas para os

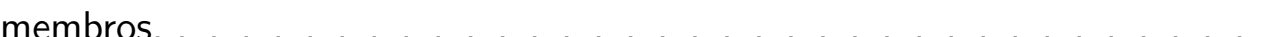

2.17 Ajuste das distribuições de $X_{\max }$. Cada linha mostra o ajuste realizado com base em um modelo de interação hadrônica. Cada coluna mostra da esquerda para a direita, o ajuste com dois, três e quatro primários. Note o valor $\mathrm{p}$ value em cada quatro resultante do ajuste. Fonte: Figura produzida pela Colaboração Pierre Auger disponível apenas para os membros. . . . . . . . .

2.18 Painel superior: frações de cada tipo de primário ajustadas às distribuições de $X_{\max }$. Painel inferior: Evolução do p-value para cada ajuste com a energia. Fonte: Figura produzida pela Colaboração Pierre Auger disponível apenas para os membros.

2.19 Abundância de cada tipo de primário em função da energia. Fonte: Figura produzida pela Colaboração Pierre Auger disponível apenas para os membros. p. 52

3.1 Espectro da radiação de fundo divido em três componentes: COB (óptico), CIB (infravermelho) e CMB (micro-ondas). Fonte: Figura extraída da referência (9).

3.2 Livre caminho médio de fótons na radiação de fundo em função da energia do fóton. A figura mostra o prospecto de estudo do Observatório CTA. Fonte: Adaptado da referência $(10) \ldots \ldots$. . . . . . . . . . . . . . . 
3.3 Medidas da radiação infravermelho de fundo. Chary (11), Gardner (12), Hauser+01 (13), Matsuura (14), Keenan (15), Lagache (9), Schlegel (16), Finkbeiner (17), Bernstein (18), Fixsen (19), Xu (20), Frayer (21), Béthermin (22), Hauser+98 (23), Levenson\&Wright (24), Berta (25), Madau (26), Fazio\&Fran (27), Madau (26), Metcalfe (28), Gorgin (11), Cambrésy (29). Fonte: Figura extraída da referência (30).

3.4 Modelos da radiação cósmica infravermelha. Malkan\&Stecker (31), Dominguéz (32), Aharonian+03 (33), Mazin\&Raue (34), Albert (35), Aharonian+06 (36), Franceschini+01 (37), Franceschini+08 (38), Gilmore (32) e Kneiske (39). Fonte: Figura extraída da referência (30).

3.5 Principais modelos de radiação cósmica infravermelha utilizados na propagação de Ultra High Energy Cosmic Ray $\left(E>10^{18} \mathrm{eV}\right)$ (UHECR). Malkan\&Stecker (31) e Dominguéz (32). Fonte: Figura extraída da referência (30). p. 60

3.6 Seção de choque da interação próton-fótons em função da energia do fótons no sistema de referência do próton $E_{\gamma}^{S R P C}=\epsilon^{\prime}$. Fonte: Figura extraída da referência (40) . . . . . . . . . . . . . . . . . . . . . .

3.7 Seção de choque da interação núcleo de ferro-fótons em função da energia do fótons no sistema de referência do próton $E_{\gamma}^{S R P C}=\epsilon^{\prime}$. Fonte: Figura extraída da referência (40).

3.8 Fluxo de raios cósmicos e raios gamas na Terra em função do logaritmo da energia para Pictor A emitindo um espectro de lei de potência com características dada no texto. Linhas cheias correspondem à injeção de próton e linhas tracejadas à injeção de núcleo de ferro. Fonte: Figura extraída da

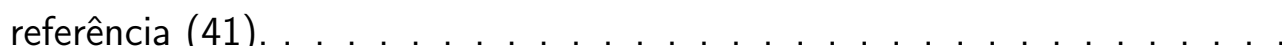

3.9 Limite superior de UHECR produzidos por Pictor A de acordo com cálculo baseado no limite superior da integral do fluxo de raios gama com energia entre GeV-TeV medidos pelos telescópios H.E.S.S.. Os pontos corresponder ao espectro de energia medido pelo Observatório Pierre Auger. Coluna da esquerda: Figuras (a), (c), e (e) correspondem a prótons primários. Coluna da direita: Figuras (b), (d), e (f) correspondem a núcleos de ferro primários.

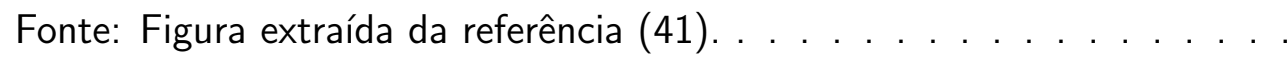


3.10 Limite superior na luminosidade de prótons emitidos por Pictor A em função do índice espectral de injeção. Os cálculos foram obtidos com base no limite do fluxo integral de raios gama com energia $\mathrm{GeV}-\mathrm{TeV}$ medidos pelo H.E.S.S.. As energias de corte consideradas são $\log \left(E_{\text {cut }} / \mathrm{eV}\right)=20,20.25,20.5,20.75$, e 21. Fonte: Figura extraída da referência (41).

3.11 Limite superior da integral do fluxo de raios gama ( $I_{\gamma}^{U H E C R}$ a $\left.95 \% \mathrm{CL}\right)$ em função da distância da fonte. As linhas vermelhas com quadrados abertos correspondem a núcleos de ferro primário. As linhas azuis com quadrados fechados correspondem a prótons primários. As setas correspondem aos dados medidos pelos observatórios de raios gama multiplicados pela exposição $\left(W_{s}\right)$ correspondente. Para todos os casos consideramos $\alpha=2.4 \mathrm{e}$ $E_{c u t}=Z \times 10^{10.5} \mathrm{eV}$. Em cada gráfico a legenda mostra o Observatório que mediu os raios gamas (FERMI-LAT), o Observatório de UHECR (Auger ou TA) e a energia dos fótons usada para calcular o limtes no fluxo. Fonte:

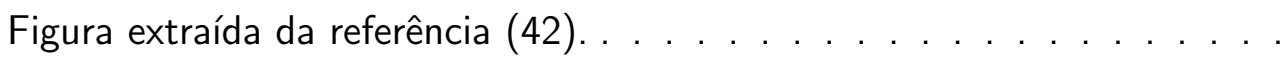

4.1 Gráfico de Hillas. São mostrados a intensidade do campo magnético e o raio da fonte aceleradora para diversos tipos de objetos astrofísicos. As linhas diagonais são a solução da equação 4.0.2 para próton (linha cheia) e núcleo de ferro (linha tracejada) com $10^{20} \mathrm{eV}$. Fonte: Figura extraída da referência (43) . . . . . . . . . . . . . . . . . . . .

4.2 Representação esquemática do espectro de energia proposto pela referência (44). Quatro curvas são mostradas: 1) explosão de supernovas no meio interestelar (fase Sedov), 2) explosão de supernova no vento estelar (Wolf-Rayet), 3) componente polar da explosão da supernova e 4) componente extragaláctica (rádio galáxias). Fonte: Figura extraída da referência (45). . . . . . . . . p. 77

4.3 Ajuste da forma do espectro de energia proposto na referência (44) ao dados do Observatório Pierre Auger. Fonte: Figura extraída da referência (46). p.79

4.4 Ajuste do espectro de energia medido pelos Observatórios KASCADE, KASCADEGrande e Pierre Auger. Os números na parte superior da figura mostram um medida do erro do ajuste (Modelo - Dados)/(Erro experimental). Fonte: Figura extraída da referência $(47) \ldots \ldots \ldots$. . . . . . . . . . . . 80 
4.5 Fluxo de próton $(H)$ em função da energia. Os dados de CREAM (48, 49), AMS-02 (50), $\operatorname{KASCADE}(51,52)$ e $\operatorname{KASCADE-Grande~}(53,54)$ são mostrados juntamente como modelo considerado. Fonte: Figura extraída da referência (45) . . . . . . . . . . . . . . . . . . . . . .

4.6 Fluxo de núcleos de hélio em função da energia. Os dados de CREAM (48, 49) e AMS-02 (50) são mostrados juntamente como modelo considerado. Todas as quebras e cortes de energia seguem o modelo de dependência com a rigidez. Fonte: Figura extraída da referência (45) . . . . . . . . . . . . . .

4.7 Fluxo de núcleos com massa intermediária ( $\mathrm{He}, \mathrm{CNO}, \mathrm{NeS}$ and $\mathrm{CIMn}$ ) em função da energia. Dados de $\operatorname{CREAM}(48,49), \operatorname{KASCADE}(51,52)$ e $\operatorname{KASCADE}-G r a n d e(53,54)$ são mostrados juntamente como modelo considerado. Todas as quebras e cortes de energia seguem o modelo de dependência com a rigidez. Fonte: Figura extraída da referência (45). . . . .

4.8 Fluxo de núcleos de ferro em função da energia. Dados de $\operatorname{CREAM}(48,49)$, $\operatorname{KASCADE}(51,52)$ e KASCADE-Grande $(53,54)$ são mostrados juntamente como modelo considerado. "This model - Rigidity Dependency" é usado para identificar a hipótese na qual $E_{2}^{\text {cutoff-e }}=Z \times E_{2}^{\text {cutoff-H }}$ para todos os elementos. "This model - Fe excess" é usado para identificar a hipótese na qual $E_{2}^{\text {cutoff-e }}=Z \times E_{2}^{\text {cutof } f-H}$ para todos os elementos exceto Fe. Fonte: Figura extraída da referência $(45) \ldots \ldots$. . . . . . . . . . . . . .

4.9 Fluxo de todas as partículas em função da energia. Dados de CREAM (48, 49), AMS-02 (50), KASCADE (51, 52), KASCADE-Grande $(53,54)$ e Auger (5) são mostrados juntamente como modelo considerado. A extrapolação para altas energias foi feita usando o deslocamento proposto na referência (47). "This model - Rigidity Dependency" é usado para identificar a hipótese na qual $E_{2}^{\text {cutoff-e }}=Z \times E_{2}^{\text {cutoff-H }}$ para todos os elementos. "This model - Fe excess" é usado para identificar a hipótese na qual $E_{2}^{\text {cutoff-e }}=Z \times E_{2}^{\text {cutoff-H }}$ para todos os elementos exceto Fe. Fonte:

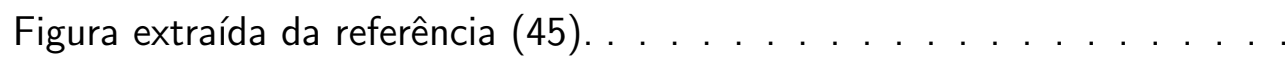


4.10 Média da profundidade do máximo do chuveiro $\left(\left\langle X_{\max }\right\rangle\right)$ em função da energia. Dados do Observatório Pierre Auger são mostrados (55). "This model - Rigidity Dependency" é usado para identificar a hipótese na qual $E_{2}^{\text {cutoff-e }}=Z \times E_{2}^{\text {cutoff-H }}$ para todos os elementos. "This model - Fe excess" é usado para identificar a hipótese na qual $E_{2}^{\text {cutoff-e }}=Z \times E_{2}^{\text {cutoff }-H}$ para todos os elementos exceto Fe. Fonte: Figura extraída da referência (45). p. 84

4.11 Dispersão da profundidade do máximo do chuveiro $\left(\operatorname{RMS}\left(X_{\max }\right)\right)$ em função da energia. Dados do Observatório Pierre Auger são mostrados (55). "This model - Rigidity Dependency" é usado para identificar a hipótese na qual $E_{2}^{\text {cutoff-e }}=Z \times E_{2}^{\text {cutoff-H }}$ para todos os elementos. "This model - Fe excess" é usado para identificar a hipótese na qual $E_{2}^{\text {cutoff-e }}=Z \times E_{2}^{\text {cutoff-H }}$ para todos os elementos exceto Fe. Fonte: Figura extraída da referência (45). p. 84

5.1 Visão artística do Observatório CTA. Fonte: Figura produzida pela Colaboração CTA disponível somente para seus membros. . . . . . . . . . . . . p p.88

5.2 Ilustração das potencialidades do CTA. A figura mostra o fluxo de raios gamas em função da distância da fonte. Fonte: Ilustração adaptada da

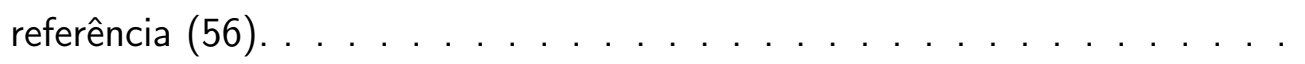

5.3 Protótipo do telescópio médio do CTA instalado em Berlim. A parte cinza foi elaborada, desenvolvida e construída por nós em colaboração com a empresa Orbital Engenharia. Fonte: Foto tirada por Ronald Shellard. 


\section{LISTA DE TABELAS}

2.1 Resumo das potencialidade do Observatório Pierre Auger. A coluna nomeada Surface Detector - Nomenclatura da Colaboração Auger (SD) refere-se a medidas feitas apenas com os detectores de superfície. A coluna SD e Fluorescence Detector - Nomenclatura da Colaboração Auger (FD) refere-se aos eventos medidos simultaneamente com os detectores de superfície e de fluorescência (medida híbrida). . . . . . . . . . . . . . . . .

3.1 A coluna 1 mostra o nome das fontes. A coluna 2 e 3 são os dados retirados das referências $(57,58)$ que mostram a distância da fonte à Terra e o limite superior do fluxo de raios gama $(E>0.1 \mathrm{GeV})$ com $95 \% \mathrm{CL}$ medido pelo FERMI-LAT. A coluna 4 mostra o limite superior na luminosidade de prótons calculado para cada fonte. A coluna 5 mostra o limite superior da luminosidade total de UHECR para os casos em que foi possível fazer o cálculo. p. 71

3.2 A coluna 1 mostra o nome das fontes. A coluna 2 e 3 são os dados retirados das referências (59-61) que mostram a distância da fonte à Terra e o limite superior do fluxo de raios gama com 99\% CL VERITAS, 95\% CL MAGIC e 99\%CL H.E.S.S. acima de $E_{t h}$. A coluna 4 mostra a energia de limiar das medidas $\left(E_{t h}\right)$. A coluna 5 mostra qual observatório detectou a fonte. A coluna 6 mostra o limite superior na luminosidade de prótons calculado para cada fonte. A coluna 7 mostra o limite superior da luminosidade total de UHECR para os casos em que foi possível fazer o cálculo.

3.3 A coluna 1 mostra o nome das fontes. A coluna 2 e 3 são os dados retirados das referências $(57,58)$ que mostram a distância da fonte à Terra e o limite superior do fluxo de raios gama $\left(10<E_{\gamma}<100 \mathrm{GeV}\right)$ com 95\% CL tais como medido pelo FERMI-LAT. A coluna 4 mostra o limite superior na luminosidade de prótons calculado para cada fonte. Para essas fontes não foi possível calcular um limite no fluxo de UHECR. 



\section{Abreviações}

AGN Active Galactic Nuclei. 36-38, 71

AugerPrime Upgrade do Observatório Pierre Auger. 25

CMB Cosmic Microwave Background - Radiação Cósmica de Micro-ondas. 52, 53

CTA Cherenkov Telescope Array. 25

FD Fluorescence Detector - Nomenclatura da Colaboração Auger. 29, 34, 39

HEAT High Elevation Atmospheric Telescope. 29

MC Monte Carlo. 35, 60

SD Surface Detector - Nomenclatura da Colaboração Auger. 29, 34

UHECR Ultra High Energy Cosmic Ray $\left(E>10^{18} \mathrm{eV}\right) .24,25,52,56-62,64,69-71,74$, 82,85 



\section{SUMÁRIO}

1 Introdução $\quad$ p. 27

2 Detecção de Raios Cósmicos de Altíssimas Energias $\quad$ p. 31

2.1 O Observatório Pierre Auger . . . . . . . . . . . . . . . . . p. 33

2.1.1 Potencialidades do Observatório Pierre Auger . . . . . . . . . . . p. 35

2.1.2 Principais Resultados do Observatório Pierre Auger . . . . . . . . . p. p.40

2.1.2.1 Composição e espectro de massas . . . . . . . . . . . . p. p. 44

2.2 Status Experimental . . . . . . . . . . . . . . . . . p. 53

3 Propagação de Raios Cósmicos de Altíssimas Energias p. 55

3.1 Radiação de fundo no Universo . . . . . . . . . . . . . . p. 56

3.2 Principais interações de UHECR com a radiação de fundo . . . . . . . . . p. 61

3.3 UHECR produzindo fótons de $\mathrm{GeV}-\mathrm{TeV} \ldots \ldots \ldots$ p. 63

3.3.1 Limite na luminosidade de fonte pontuais de UHECR . . . . . . . p. 64

4 Geração de Raios Cósmicos de Altíssimas Energias p.73

4.1 Modelo Stanev, Biermann e Gaisser - $1993 \ldots \ldots$. . . . . . . . . . . . 76

4.1 .1 Centaurus A . . . . . . . . . . . . . . . . . . . . 78

4.1.2 UHECR vindos de Cen $A \ldots \ldots \ldots$ p.78

4.1.3 Via Láctea + Cen A: as fontes de raios cósmicos . . . . . . . . . p. 79

5 Perspectivas futuras $\quad$ p. 85

5.1 AugerPrime . . . . . . . . . . . . . . . p. 86

5.2 CTA - Cherenkov Telescope Array . . . . . . . . . . . . . p. 87 
REFERÊNCIAS

p. 91 


\section{CAPÍtulo 1}

\section{Introdução}

Os fenômenos da natureza estão limitados por dois valores de energia: a escala de Planck $\left(E \sim 10^{28} \mathrm{eV}\right.$ ou $\left.l \sim 10^{-35} \mathrm{~m}\right)$ e a escala de Hubble $\left(l \sim 10^{26} \mathrm{~m}\right.$ ou $\left.E \sim 10^{-32} \mathrm{eV}\right)$. Nossa tarefa como pesquisadores de ciências naturais é explicar os fenômenos ocorrendo neste intervalo de energia que vai de $10^{-32}$ a $10^{28} \mathrm{eV}$, ou seja, 60 ordens de grandeza. Nossa ciência não quer pouco.

Através do nosso trabalho, buscamos contribuir com a descrição cada vez com melhor precisão os fenômenos entres estas duas escalas limites de energia usando o menor número de suposições ad hoc. No tocante ao estudo do Universo, temos pouquíssima informação disponível. Toda o sinal que recebemos do Cosmo está concentrado na faixa de energia que vai de $10^{-10}$ a $10^{20} \mathrm{eV}$ de forma que temos 30 ordens de grandeza de informação disponível para explicar 60 ordens de grandeza de fenômenos.

Astrofísica de partículas é a pesquisa realizada com partículas provenientes do Cosmo. Usando uma divisão didática de chamar de partícula tudo o que tem energia maior que a massa de um par elétron-pósitron, podemos dizer que astrofísica de partículas é a ciência que estuda o sinal produzido fora da Terra com energia superior a $10^{6} \mathrm{eV}$. Ela pode ser vista com dois vieses que se alternam e se fundem ao longo da história. Objetos astrofísicos podem acelerar partículas a energia superior a produzida em aceleradores humanos e portanto partículas cósmicas permitem estudar as interações fundamentais. Por outro lado, as partículas cósmicas trazem informações importantes dos mecanismos astrofísicos operando em objetos altamente energéticos e assim servem como uma ferramenta valiosa na tentativa de descrever a operação desses astros.

Astrofísica de partículas é uma das poucas linhas de pesquisa que toca as escalas de Planck e as escalas de Hubble permitindo estudos sobre os extremos microscópicos (partículas elementares) e macroscópicos (cosmologia). Esta é umas das características que me fascina nesta área. Ao englobar uma abrangência imensa de conceitos da física, a Astrofísica de Partícula tem como característica intrínseca estar sempre próxima das questões centrais da física. 
A escrita desta tese de livre-docência se dá onze anos após a defesa da minha tese de doutorado (2004) e sete anos após minha contratação no IFSC-USP (2008). Muito se passou em astrofísica de partículas neste período e muito ainda há para ser explicado.

Nesta última década, vivemos uma transição de fase no estudo de raios cósmicos. Passamos de um estado puramente especulativo para outro de ciência de precisão. A melhoria das técnicas experimentais, a instalação de grandes áreas coletoras e a operação continuada de novos observatórios, trouxeram a pesquisa em UHECR para um novo nível de confiança. Neste sentido, foi uma década muito positiva para a Astrofísica de Partículas.

No entanto, também vivemos uma década de algumas esperanças frustradas.Por inúmeras questões econômicas e político-científicas não foi construído o sítio norte do Observatório Pierre Auger que traria um avanço significativo ao possibilitar o estudo completo do céu. Vimos ao longo desta década o nascimento do sonho da astronomia de partículas carregadas que até o momento tem sido frustrado já que nenhuma fonte de UHECR foi inequivocamente detectada. Acompanhamos os esforços de inaugurar a instalação de experimentos de UHECR no espaço serem postergados juntamento com o inicio de uma nova era por eles representada.

Esta tese se insere neste contexto e tem como objetivo mostrar nossa contribuição para esta área de pesquisa. Não pretendemos com a tese fazer um compêndio de Astrofísica de Partículas pois para tal já existem os livros e artigos de revisão. A tese traz uma compilação de três linhas de pesquisa por nós abordadas nos últimos anos. As três linhas de pesquisa foram desenvolvidas depois da minha contratação no IFSC-USP. Nenhum resultado científico novo será apresentado nesta tese que já não tenha sido publicado em revistas científicas. Com essa tese, temos o intuito de reorganizar os trabalho já publicados e dispô-los de forma mais contínua e conexa do que nas publicações sucintas de revistas especializadas. O leitor verá que os gráficos foram todos retirados das publicações anteriores, suas legendas não foram atualizadas ou traduzidas.

Por outro lado, nem tudo o que publicamos e produzimos nos últimos anos faz parte desta tese. Escolhi três linhas de trabalho que ilustram nosso envolvimento em todos os aspectos da pesquisa em Astrofísica de Partículas: experimental e teórico, instrumentação e análise de dados, experimentos em funcionamento e em planejamento. Cada capítulo serve como exemplo da pesquisa que realizamos dentro daquele assunto. Uma visão completa de todos os trabalhos realizados pode ser vista através das publicações listadas no meu Curriculum Vitae.

No capítulo 2, ilustramos nosso envolvimento recente com o Observatório Pierre Auger e a análise de dados de composição. No capítulo 3, mostramos uma linha de trabalho que explora a propagação de UHECR e no capítulo 4 discutimos um modelo de aceleração de partículas em 
radio galáxias. No último capítulo 5 discutimos perspectivas futuras relacionadas ao Upgrade do Observatório Pierre Auger (AugerPrime) e ao Cherenkov Telescope Array (CTA). 


\section{Detecção de Raios Cósmicos de Altíssimas Energias}

A detecção de raios cósmicos com energia acima de $10^{17} \mathrm{eV}$ exige um complicado arranjo experimental e dedicação continuada de monitoramento. O fluxo de partículas cósmicas com estas energias atingindo a Terra é menor do que uma partícula por $\mathrm{km}^{2}$ por ano o que necessariamente implica na necessidade de grandes áreas de deteç̧ão $\left(>1000 \mathrm{~km}^{2}\right)$. Instrumentar grandes áreas de detecção requisita detectores controlados remotamente, energeticamente autônomos e de baixa manutenção.

Mesmo com grandes áreas instrumentadas $\left(\sim 1000 \mathrm{~km}^{2}\right)$, um observatório necessita tomar dados por vários anos para detectar um número estatisticamente significativo de eventos. Assim, os detectores instalados em regiões amplas e inóspitas requerem calibração precisa e estabilidade extremas ao longo de vários anos.

O sinal a ser medido por um experimento de raios cósmicos de altas energias é o produto das interações da partícula cósmica com a atmosfera chamado de chuveiro atmosférico. $O$ chuveiro atmosférico gerado por uma partícula primária* com energia superior a $10^{17} \mathrm{eV}$ produz um sinal amplo e intenso. O chuveiro atmosférico é o fenômeno que amplifica espacialmente o sinal da partícula cósmica, facilitando a detecção do evento, ao mesmo tempo que dilui a informação, reduzindo a densidade do sinal.

Ao atingirem baixas altitudes atmosféricas $(<3 \mathrm{~km})$, as partículas geradas no chuveiro cobrem uma região com área maior que alguns $\mathrm{km}^{2}$. Detectores espalhados pela superfície terrestre podem medir a passagem dessas partículas e amostrarem o desenvolvimento lateral do chuveiro. Ao longo do caminho das partículas na atmosfera, radiação Čerenkov e de fluorescência é gerada em abundância. Telescópios sensíveis podem detectar a radiação produzida e assim medir o desenvolvimento longitudinal do chuveiro.

Vários detectores foram desenvolvidos para estudar raios cósmicos, entre eles, cintiladores, detectores à gás e detectores Čerenkov, alguns deles encontraram aplicações variadas, inclusive

\footnotetext{
* O termo partícula primária é usado para designar o raio cósmico que deu origem a um chuveiro atmosférico sendo portanto a partícula primária deste chuveiro.
} 
em medicina. Detectores de luz Čerenkov são conhecidos com um dos mais estáveis detectores de superfície para medir chuveiros atmosféricos. Estes detectores são constituídos por um reservatório de volume considerável $\left(\sim\right.$ dezenas de $\mathrm{m}^{3}$ ) preenchido com água através do qual as partículas dos chuveiros viajam com velocidade maior do que a da luz na água. A polarização não homogênea causada pela passagem das partículas gera o conhecido efeito Čerenkov de emissão de radiação concentrada em um cone. Os detectores Čerenkov são instrumentados para detectar a luz produzida pelas partículas como sinal da passagem de um chuveiro atmosférico.

As partículas produzidas no chuveiro excitam os módulos vibracionais e rotacionais das moléculas do gás nitrogênio no ar que ao voltarem ao seu estado fundamental, emitem radiação com comprimento de onda entre 300 e $450 \mathrm{~nm}$. Um chuveiro iniciado por uma partícula com energia superior a $10^{17}$ gera luz de fluorescência com intensidade factível de ser detectada com boa razão sinal/ruído em noites limpas e sem lua (62).

Vários experimentos dedicados à medir raios cósmicos de altíssima energia operaram entre as décadas de 1980 e 2000, entre eles cito: Haverah Park, Yakutsky, Fly's Eye, CASA-MIA, HiRes e AGASA. Haverah Park e Fly's Eye foram os pioneiros em utilizar detectores de luz Čerenkov na água e fluorescência na atmosfera, respectivamente. Ambas as técnicas foram postas para operar em conjunto no Observatório Pierre Auger a partir de 2004.

Na década de 1990, os dois principais experimentos em operação eram os experimentos HiRes e AGASA com 167 e 667 km² sr ano de exposição para raios cósmicos com energia da ordem de $10^{20} \mathrm{eV}$, respectivamente (63). O número de eventos com energia acima de $10^{20} \mathrm{eV}$ medidos por ambos os observatórios era baixo ( 1 e 6 , respectivamente (63)) e insuficientes para responder as principais questões em aberto sobre a origem dessas partículas. Ademas, a análise dos dados desses experimentos revelavam discordâncias cruciais a respeito do fluxo de partículas e da distribuição das direções de chegada. HiRes utilizava a técnica de detecção de luz de fluorescência. AGASA utilizava detectores de superfície (cintiladores) espalhados por $100 \mathrm{~km}^{2}$. Neste década, o status experimental era ditado por dois experimentos com área limitada de detecção resultando em um baixo número de eventos medidos que no entanto levaram a conclusões discrepantes: era o prenúncio de um novo experimento que deveria inaugurar uma nova era em astrofísica de partículas.

A comunidade internacional reconheceu que a região de altíssimas energias só poderia ser estudada de forma adequada por um experimento com pelo menos $3000 \mathrm{~km}^{2}$ de área que utilizasse ao mesmo tempo detectores do desenvolvimento longitudinal e lateral do chuveiro atmosférico combinando assim as técnicas utilizadas por HiRes e AGASA. Era o nascimento 
do Observatório Pierre Auger.

\subsection{O Observatório Pierre Auger}

O Observatório Pierre Auger foi proposto por Jim Cronin e Alan Watson no início da década de 1990. Em 1996, uma colaboração internacional foi formada com o intuito de estudar a potencialidade e optimizar a configuração de um observatório híbrido com detectores do desenvolvimento lateral e longitudinal do chuveiro. Em 2000, um arranjo de 32 detectores de superfície (detectores de Čerenkov na água) e 2 telescópios de fluorescência começaram a ser instalados nas redondezas da cidade de Malargüe, Argentina.

A configuração final do Observatório foi inaugurada em 2008 contendo 1660 detectores de Čerenkov e vinte e quatro telescópios de fluorescência. Posteriormente, outros três telescópios de alta elevação foram instalados bem como um arranjo mais denso de 61 detectores. Dados de interesse científico começaram a ser medidos deste 2004 com as instalações parciais do Observatório.

A figura 2.1 mostra o arranjo de detectores de superfície e o campo de visão dos telescópios de fluorescência. Os detectores de superfície ${ }^{\dagger}$ constituídos basicamente por um tanque cheio com 12 mil litros de água e três fotomultiplicadoras que medem a luz Čerenkov produzidas pela passagem das partículas na água. Os detectores estão dispostos em uma rede regular com espaçamento de $1.5 \mathrm{~km}$ e são alimentados por um sistema de painéis solares e baterias. Os dados são enviados de cada tanque para uma central de aquisição através de uma conexão sem fio, tornando assim cada tanque um detector autônomo.

Vinte e sete telescópios de luz fluorescente estão totalmente operacionais no sítio sul do Observatório Auger. Vinte e quatro destes telescópios cobrem $30^{\circ}$ em azimute e de 0 a $30^{\circ} \mathrm{em}$ elevação. Outros três telescópios formam o arranjo denominado High Elevation Atmospheric Telescope (HEAT) e cobrem $30^{\circ}$ em azimute e de 30 a $60^{\circ}$ em elevação sob um arranjo denso de detectores de superfície com espaçamento de $750 \mathrm{~m}$ cobrindo uma região de $23.5 \mathrm{~km}^{2}$.

O uso de ambas as técnicas simultaneamente resulta na redução de erros sistemáticos de medida e possibilita calibrações cruzadas entre as distintas técnicas. Sobretudo a combinação de uma área fixa de detecção oferecida pelos detectores de superfície e uma medida calo-

\footnotetext{
$\dagger$ A colaboração Auger utiliza a nomenclatura SD para designar os detectores de superfície e FD para os telescópios de fluorescência
} 
rimétrica da energia feita com os telescópio oferece uma capacidade única ao Observatório Auger. Além disso, o estudo do desenvolvimento longitudinal do chuveiro possível graças aos telescópios de fluorescência dão acesso à parâmetros importantes para a determinação da massa da partícula primária.

Os detectores de superfície operam 100\% do tempo e para chuveiros com energia superior a $3 \times 10^{18} \mathrm{eV}$ tem uma área de coleção determinada apenas pela soma da área dos hexágonos desenhado pelas estações ativas. Restrito à operar em noites sem lua e com boas condições atmosféricas, os telescópio de fluorescência tomam dados em aproximadamente $12 \%$ do tempo. No entanto, os telescópios fazem uma medida calorimétrica do desenvolvimento do chuveiro

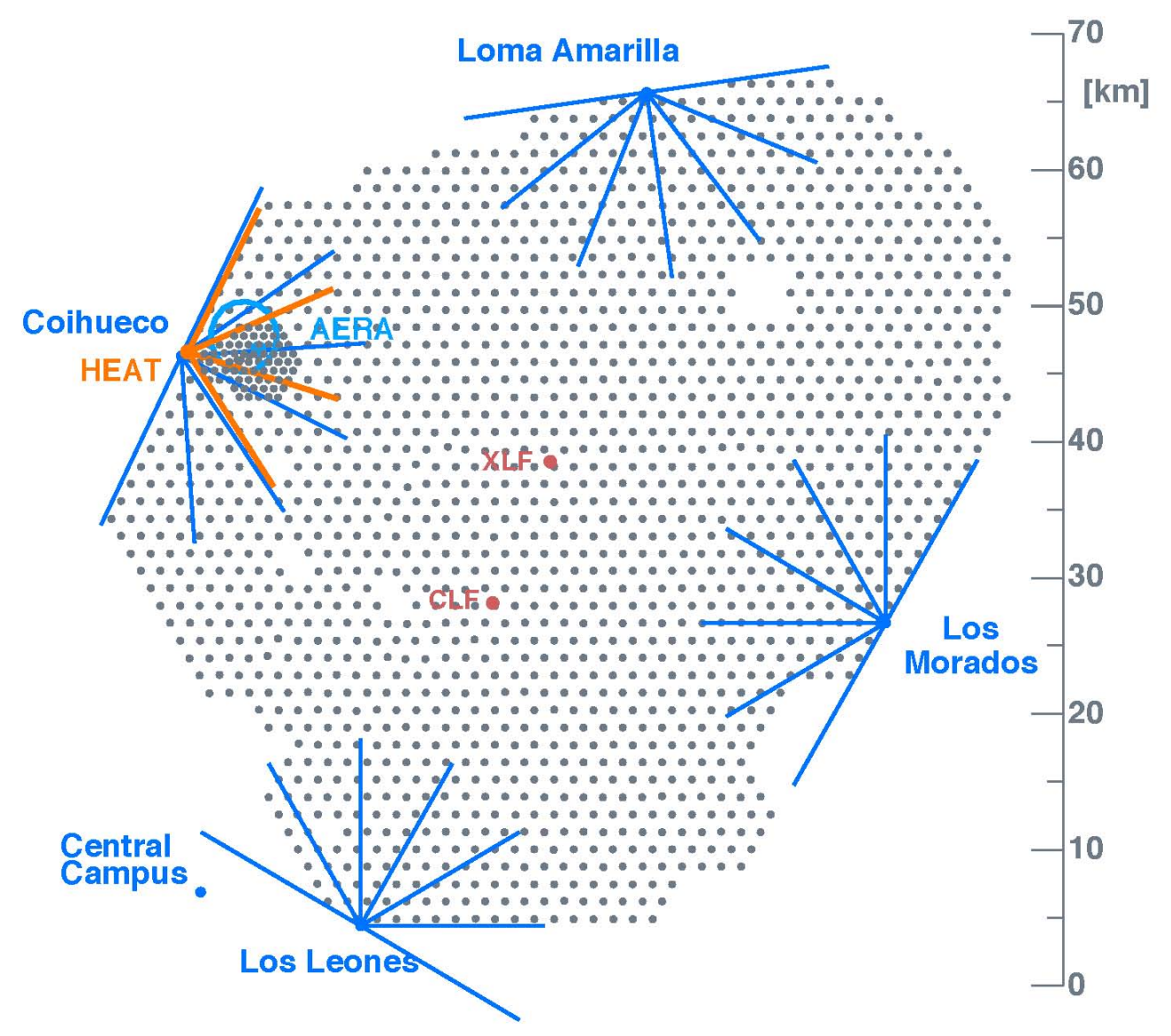

Figura 2.1 - Mapa do Observatório Auger. Os pontos mostram os detectores de superfície. As linhas azuis e vermelhas representam o campo de visão de cada telescópio de fluorescência. Dois pontos vermelhos no meio do arranjo de detectores de superfície: XLF (eXtreme Laser Facility) e CLF (Central Laser Facility), mostram a posição de instalação de monitoramento das condições atmosféricas. O ponto denominado Central Campus mostra a posição do central de operações do Observatório na cidade de Malargüe. Fonte: Figura produzida pela Colaboração Pierre Auger disponível apenas para os membros. 
permitindo uma reconstrução da energia independente de modelos de simulação dos chuveiros atmosféricos.

\subsubsection{Potencialidades do Observatório Pierre Auger}

Os detalhes sobre os detectores e suas capacidades estão publicados na referencia (64). Limitamo-nos aqui, a fazer um breve resumo das potencialidades do Observatório.

O primeiro passo na reconstrução de um chuveiro atmosférico é a determinação da direção de chegada da partícula primária. O desenvolvimento do chuveiro na atmosfera pode ser modelado pela propagação de uma frente de onda com velocidade próxima da velocidade da luz. O tempo de disparo de cada detector de superfície e de cada pixel no telescópio de fluorescência determina uma sequência de eventos univocamente relacionada com a geometria da fonte do sinal. Usando argumentos simples de geometria, é possível calcular a direção do eixo de propagação da onda de choque que modela o chuveiro atmosférico, ou seja, a direção de propagação da partícula primária do chuveiro. A figura 2.2 mostra um esquema da detecção de um chuveiro por quatro telescópios de fluorescência e catorze detectores de superfície.

Quando ambos os detectores de superfície e de fluorescência mediram o chuveiro, a reconstrução da direção tem alta resolução. O vínculo estabelecido entre a propagação longitudinal e lateral do chuveiro restringe fortemente o ajuste da geometria de propagação da onda de choque aos tempos de disparo dos detectores de superfície e os pixel dos telescópios. Se apenas os detectores de superfície detectaram o chuveiro, o ajuste não tem a mesma precisão levando à erros maiores da determinação da direção de chegada.

A energia do chuveiro pode ser diretamente determinada pelos telescópios de fluorescência. A intensidade da luz de fluorescência gerada pela passagem do chuveiro é diretamente proporcional à energia das partículas e portanto diretamente proporcional à energia da partícula primária. A figura 2.3 mostra um exemplo da intensidade da luz medida por um telescópio em função do tempo(veja figura 2.3) e a consequente determinação da energia depositada na atmosfera em função da profundidade atmosférica. Como dito anteriormente, a integral do perfil de energia depositado na atmosfera é diretamente proporcional a energia da partícula primária.

Apenas os chuveiros medidos pelos telescópios de fluorescência tem energia determinada calorimetricamente. Os eventos medidos apenas pelo detector de superfície tem apenas seu 
desenvolvimento lateral detectado tal como mostrado na figura 2.4:Esquerda. A densidade de partículas cai rapidamente com a distância em relação ao eixo do chuveiro e a 1000 metros a flutuação é mínima (65). Usando os chuveiros detectados pelos telescópios e pelos detectores de superfície é possível estabelecer uma correlação entre a densidade de partículas a 1000 $\mathrm{m}$ do eixo do chuveiro $(\mathrm{S}(1000))$ e a energia calorimétrica determinada pelos telescópios de fluorescência $\left(E_{F D}\right)$. A figura 2.4:Direita mostra esta relação onde o valor de $\mathrm{S}(1000)$ foi convertido utilizando argumentos puramente geométricos (66) para a referência de um chuveiro incidente a $38^{\circ}\left(S_{38}\right)$.

Além de permitir uma reconstrução da energia calorimétrica que é usada para calibrar a energia dos detectores de superfície, os telescópios de fluorescência tem acesso ao mais preciso

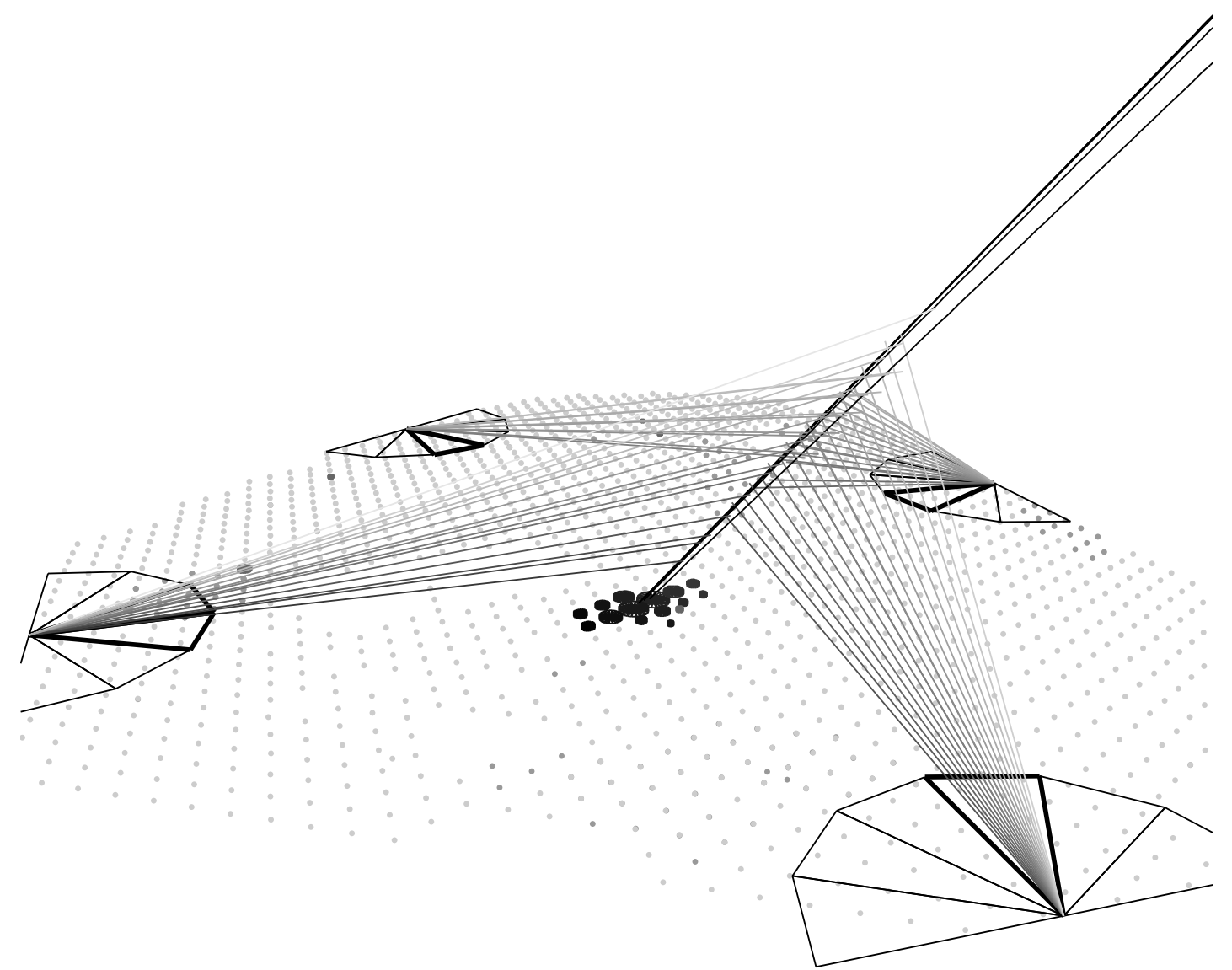

Figura 2.2 - Esquema da detecção de um chuveiro atmosférico pelo Observatório Pierre Auger. Os círculos representam todos os detectores de superfície em operação. Os círculos maiores no dentro da imagem mostram os detectores que mediram a passagem de partículas do chuveiro. As figuras sextavadas na borda do conjunto de detectores de superfície representam os telescópios de fluorescência e seus campos de visão. As linhas saindo de cada telescópio representam o centro de cada pixel disparado pelo chuveiro. As linhas verticais mostram a reconstrução da direção de chegada deste evento. Quatro reconstruções da direção são mostradas, cada uma usando os dados de um telescópio de fluorescência. Fonte: Figura produzida pela Colaboração Pierre Auger disponível apenas para os membros. 
parâmetro relacionado com a massa da partícula primária. A profundidade na qual o chuveiro atinge seu máximo $\left(X_{\max }\right)$ é diretamente proporcional à massa da partícula primária como mostrado na figura 2.5 (4). Na reconstrução do eventos medidos pelo Observatório Pierre Auger, o valor de $X_{\max }$ é calculado a partir do ajuste mostrado na figura 2.3 .

A resolução e os erros sistemáticos envolvidos na reconstrução de cada uma dos três principais parâmetros foi estuda com simulações dos detectores e com medidas cruzadas. Por exemplo, a resolução na reconstrução do valor de $X_{\max }$ pode ser estudada utilizando eventos medidos por pelo menos dois telescópios de fluorescência. Para este eventos, temos duas reconstruções de $X_{\max }$ independentes e podemos calcular diretamente a resolução na determinação deste parâmetro. A figura 2.6 mostra o resultado deste estudo. A figura mostra também a concordância entre os dados e o calculo da resolução utilizando simulação dos telescópios. Uma vez comprovada a qualidade da simulação, ela foi usada para determinar a resolução em intervalos de energia para os quais não temos muitos eventos medidos por mais de um telescópio.

A resolução de cada parâmetro reconstruído pode depender da energia, do número de detectores de superfície envolvidos, da distância do chuveiro em relação ao telescópio de fluorescência, do ângulo de inclinação do chuveiro e de muitas outras variáveis. A referência (64) faz uma compilação dos estudos de cada caso que foram publicados em diversos artigos pela Colaboração Auger. Mostramos na tabela 2.1 um valor referência das principais características das potencialidades do Observatório Pierre Auger. 

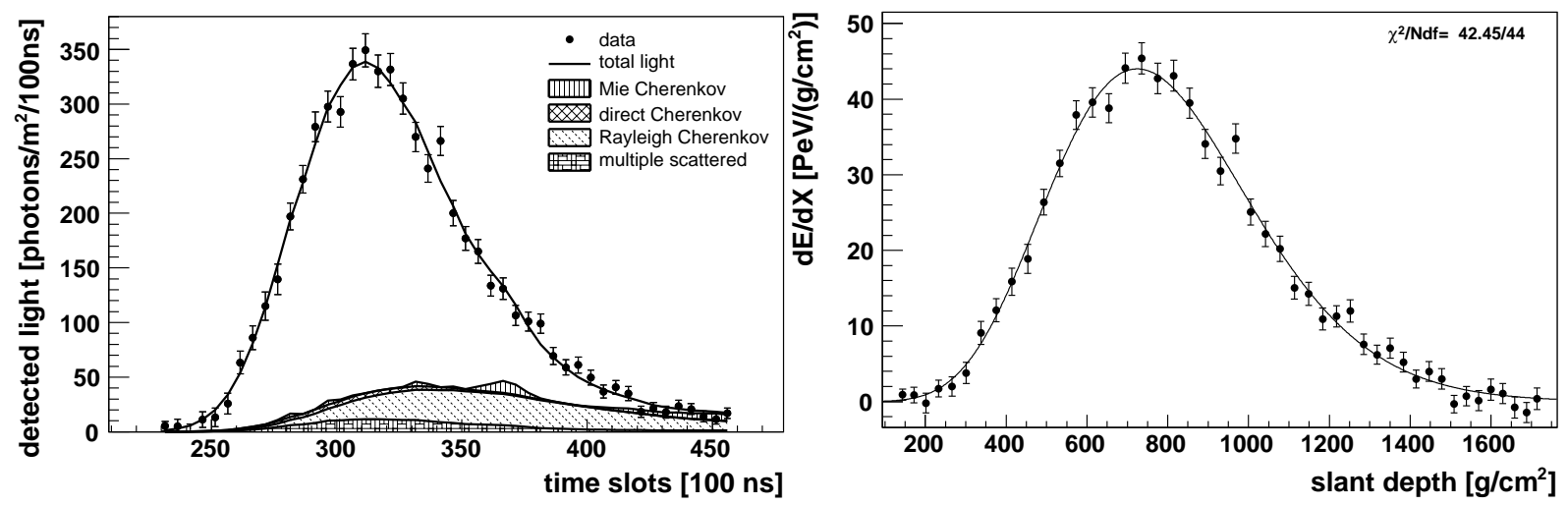

Figura 2.3 - Exemplo de um evento medido pelo telescópio de fluorescência. Esquerda: Luz detectada pelo telescópio em função do tempo. São mostradas em separado as contribuições estimadas devido efeitos de espalhamentos simples e múltiplos (Mie e Rayleigh) e contaminação por emissão Čerenkov. Direita: Perfil de energia depositada em função da profundidade atmosférica. A linha preta mostra um ajuste de uma função GaisserHillas (1) aos dados medidos. Fonte: Figura produzida pela Colaboração Pierre Auger disponível apenas para os membros.
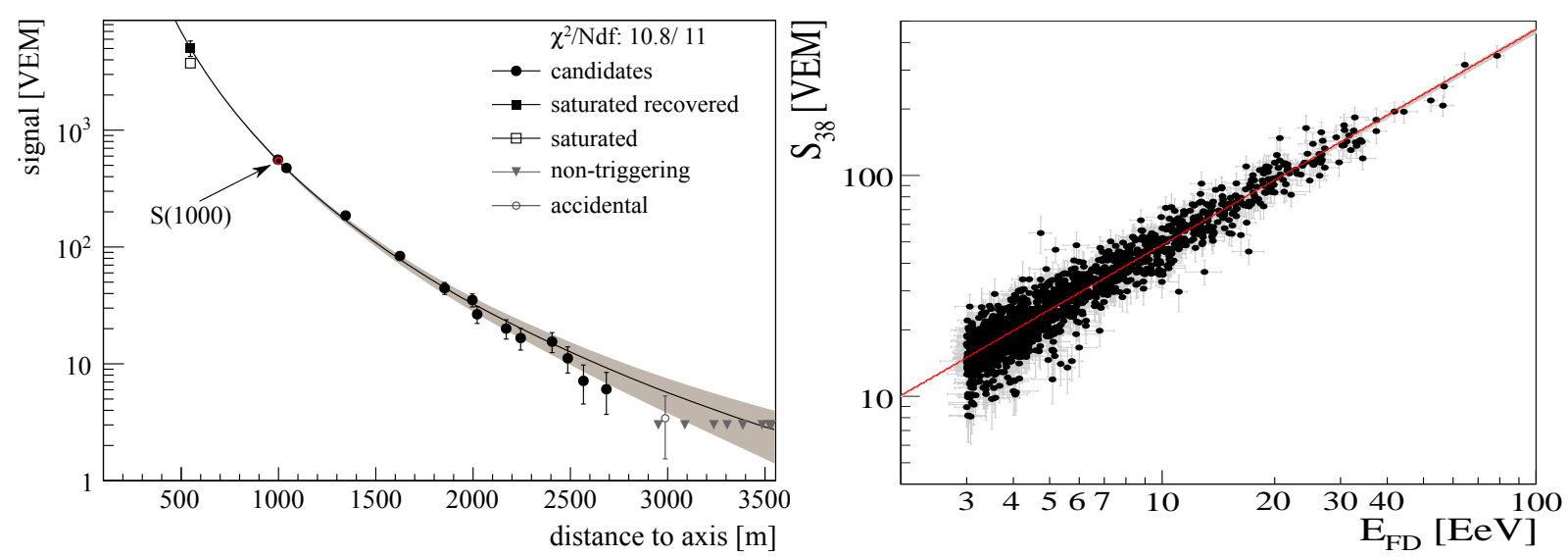

Figura 2.4 - Esquerda: Exemplo da densidade de partículas em função da distância em relação ao eixo do chuveiro. Os pontos pretos mostram os dados medidos. Os quadrados pretos mostram as estações com sinal acima do nível de saturação cujo método de reconstrução foi capaz de estimar o sinal verdadeiro. Os quadrados abertos mostram as estações com sinal acima do nível de saturação cujo método de reconstrução não foi capaz de estimar o sinal verdadeiro. Os triângulos mostram as estações na redondeza que não foram disparadas pelo chuveiro. O círculo aberto mostra uma estação classificada como não pertencente ao evento mesmo contendo sinal diferente de zero por uma verificação de coerência de disparo temporal. A linha preta mostra o ajuste de uma função ao dados medidos. A área hachurada mostra a incerteza de um sigma do ajuste da função. O ponto vermelho mostra o valor do sinal a 1000 metros do eixo do chuveiro. Direita: Calibração da energia usando eventos medidos simultaneamente pelos detectores de superfície e pelos telescópios de fluorescência através da relação entre $S_{38}$ e $E_{F D}$. Os pontos pretos são os dados medidos e a linha vermelha o ajuste de uma reta aos dados. Fonte: Figura produzida pela Colaboração Pierre Auger disponível apenas para os membros. 


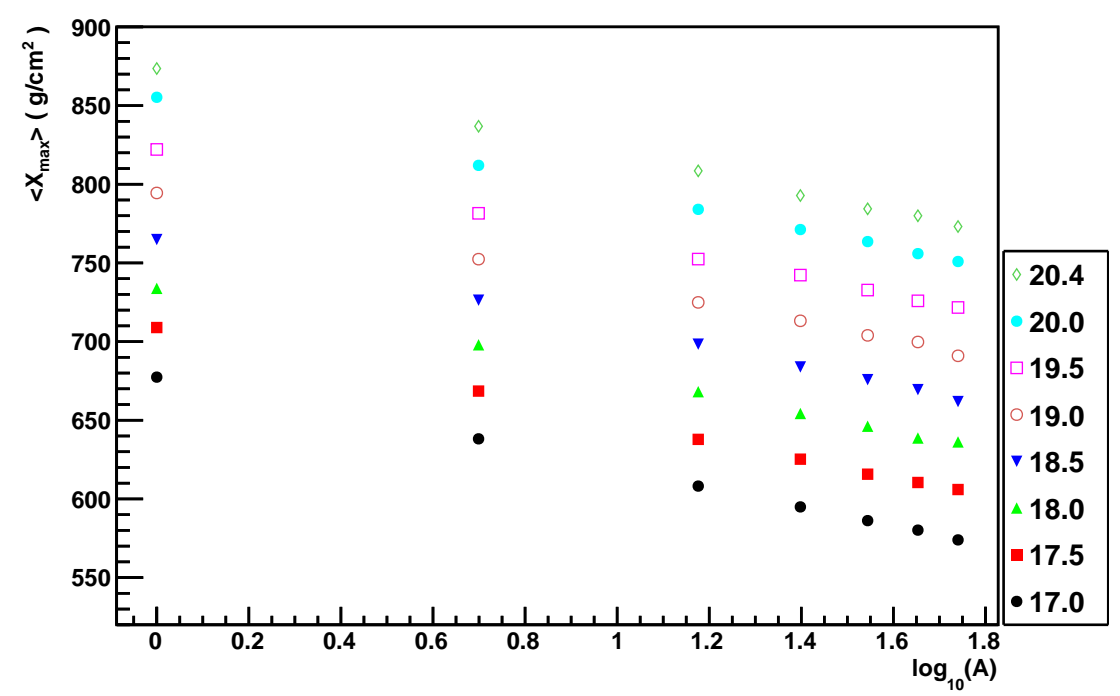

Figura 2.5 - Evolução da média da distribuição de $X_{\max }$ em função da massa da partícula primária com várias energias (cores). A figura mostra resultados de simulação de Monte Carlo de chuveiros atmosféricos utilizando o programa CORSIKA (2) como modelo de interação hadrônica Sibyll 2.1 (3). Fonte: Figura extraída da referência (4).

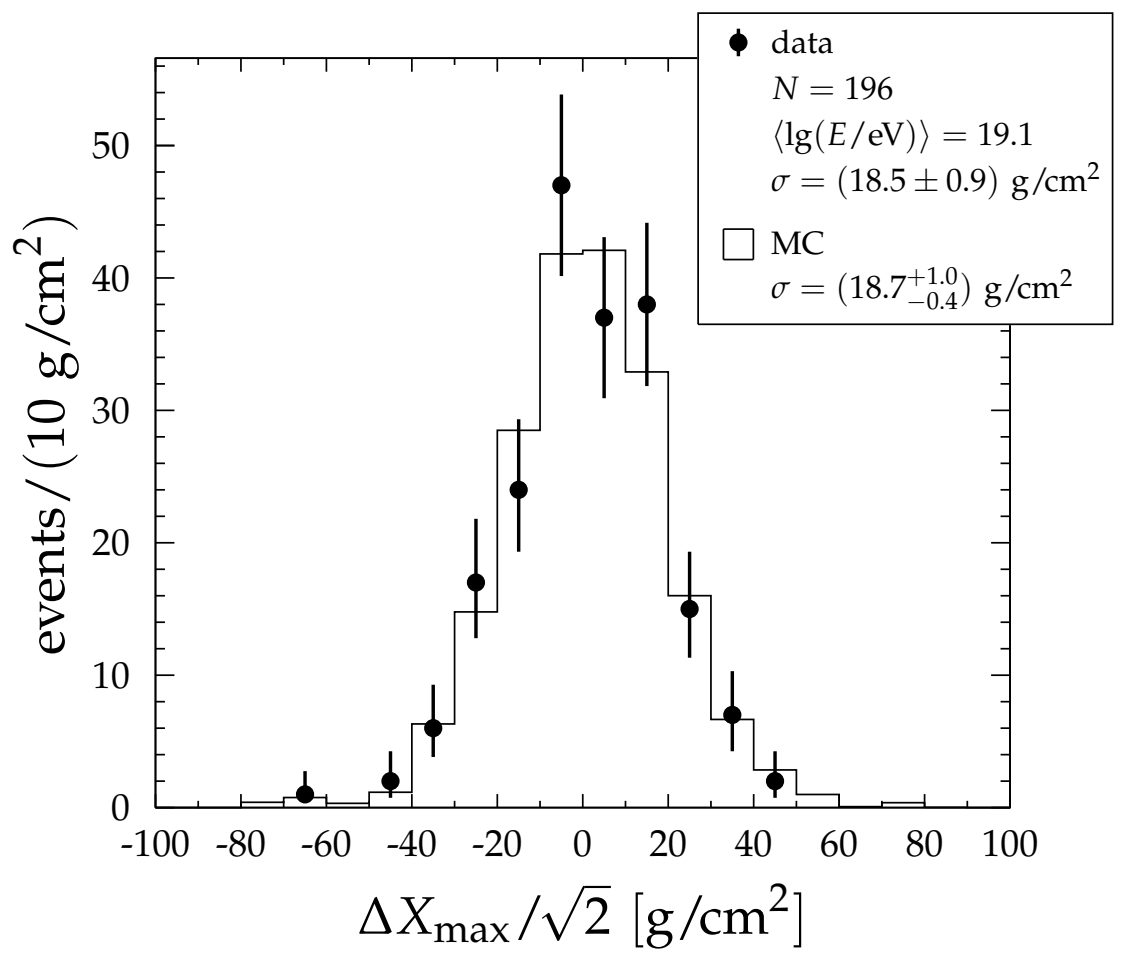

Figura 2.6 - Distribuição da diferença da reconstrução do valor de $X_{\max }$ para eventos medidos por dois telescópios de fluorescência. Os pontos pretos mostram os dados e a linha contínua o calculo utilizando simulação de MC dos detectores. Fonte: Figura produzida pela Colaboração Pierre Auger disponível apenas para os membros. 
Tabela 2.1 - Resumo das potencialidade do Observatório Pierre Auger. A coluna nomeada SD refere-se a medidas feitas apenas com os detectores de superfície. A coluna SD e FD refere-se aos eventos medidos simultaneamente com os detectores de superfície e de fluorescência (medida híbrida).

\begin{tabular}{ccc}
\hline & SD & SD e FD \\
\hline $\begin{array}{c}\text { Exposição Anual } \\
{\left[\mathrm{km}^{2} \mathrm{sr} \text { ano }\right]}\end{array}$ & $5000\left(E>3 \times 10^{18} \mathrm{eV}\right)$ & $\frac{50\left(E>10^{18} \mathrm{eV}\right)}{450\left(E>10^{19} \mathrm{eV}\right)}$ \\
\hline Resolução Angular & $0.9-1.6^{\circ}$ & $0.6^{\circ}$ \\
\hline Resolução Energia & $12-16 \%$ & $8 \%$ \\
\hline Resolução $X_{\max }$ & - & $<20 \mathrm{~g} / \mathrm{cm}^{2}$ \\
\hline
\end{tabular}

\subsubsection{Principais Resultados do Observatório Pierre Auger}

Resumimos nesta seção alguns dos principais resultados medidos pelo Observatório Pierre Auger. A primeira medida de grande importância e precisão realizada foi o espetro de energia mostrado na figura 2.7. O espectro pode ser descrito por uma lei de potência na energia com expoente 3.23 até $\log (E / e V)=18.72$ e para energias acima, por uma lei de potência na energia com expoente 2.63. Para energias ainda maiores, segue uma supressão do fluxo claramente observada acima de $\log (E / e V)=19.63$, detectada com alta significância estatística de $>20 \sigma(5,67)$.

A direção de chegada dos eventos foi estudada exaustivamente na tentativa de encontrar as fontes emissoras entretanto até o presente momento nenhuma fonte ou classe de objetos foi inequivocamente identificada. Foram realizados inúmeros estudos de correlação das direções de chegada dos eventos com fontes catalogadas como emissores em outros comprimentos de ondas (6). Da mesma forma, foram realizados estudos de autocorrelação na busca de anisotropias em diferentes escalas angulares (68). A ausência de aglomerados de eventos de altas energias foi usada para calcular um limite na densidade de fontes da ordem de $10^{-4}$ $\mathrm{Mpc}^{-3}$ dependendo da intensidade do campo magnético e de considerações a respeito da evolução e intensidade intrínseca das fontes, para mais detalhes veja a referência (68). Estudo de anisotropias de larga escala baseados em análises Rayleigh apontam para uma anisotropia de eventos com energia acima de $8 \mathrm{EeV}$ com probabilidade de $6,4 \times 10^{-5}$ de pertencer à uma distribuição isotrópica (69).

A figura 2.8 mostra a direção de chegada dos eventos com energia acima $58 \mathrm{EeV}$. A figura 
mostra como pontos pretos a direção de chegada dos eventos medidos e em zonas azuis a direção de AGNs do catálogo Swift-BAT (70). A probabilidade binomial cumulativa de que um fluxo isotrópico levaria a mesma correlação observada entre a direção de chegada dos eventos e o AGNs é de $6 \%$ levando em conta penalidades por todas as buscas. A figura 2.9 mostra o mesmo mapa considerando apenas AGNs com luminosidade maior que $10^{44} \mathrm{erg} \mathrm{s}^{-1}$ e com distância menor que $130 \mathrm{Mpc}$ da Terra. Para este conjunto, a correlação é máxima em janelas de $18^{\circ} \mathrm{com}$ probabilidade cumulativa de que um fluxo isotrópico levaria a mesma correlação observada entre a direção de chegada dos eventos e o AGNs é de $1.4 \%$ levando em conta penalidades por todas as buscas.

Outro estudo interessante mostra a correlação da direção dos eventos com Centaurus A. Mais detalhes sobre este fonte podem ser encontrados na seção 4.1.1. A figura 2.10 mostra a distribuição do número de eventos em relação a distância angular de Centaurus $A$ em comparação com predições de isotropia. Note que para distâncias angulares intermediarias (15 a 20 graus) há um excesso de eventos com significância superior da ordem de $3 \sigma$.

Importantes buscas por neutrinos e fótons com energia acima de $10^{18} \mathrm{eV}$ foram realizadas pela Colaboração Auger porém nenhum evento iniciados por estas partículas foi encontrada no conjunto de dados medidos. A ausência de neutrinos e fótons permitiu o cálculo de restritivos limites superiores no fluxo que tem sido utilizado para eliminar modelos teóricos de geração destas partículas. Veja referências $(71,72)$ para mais detalhes. 


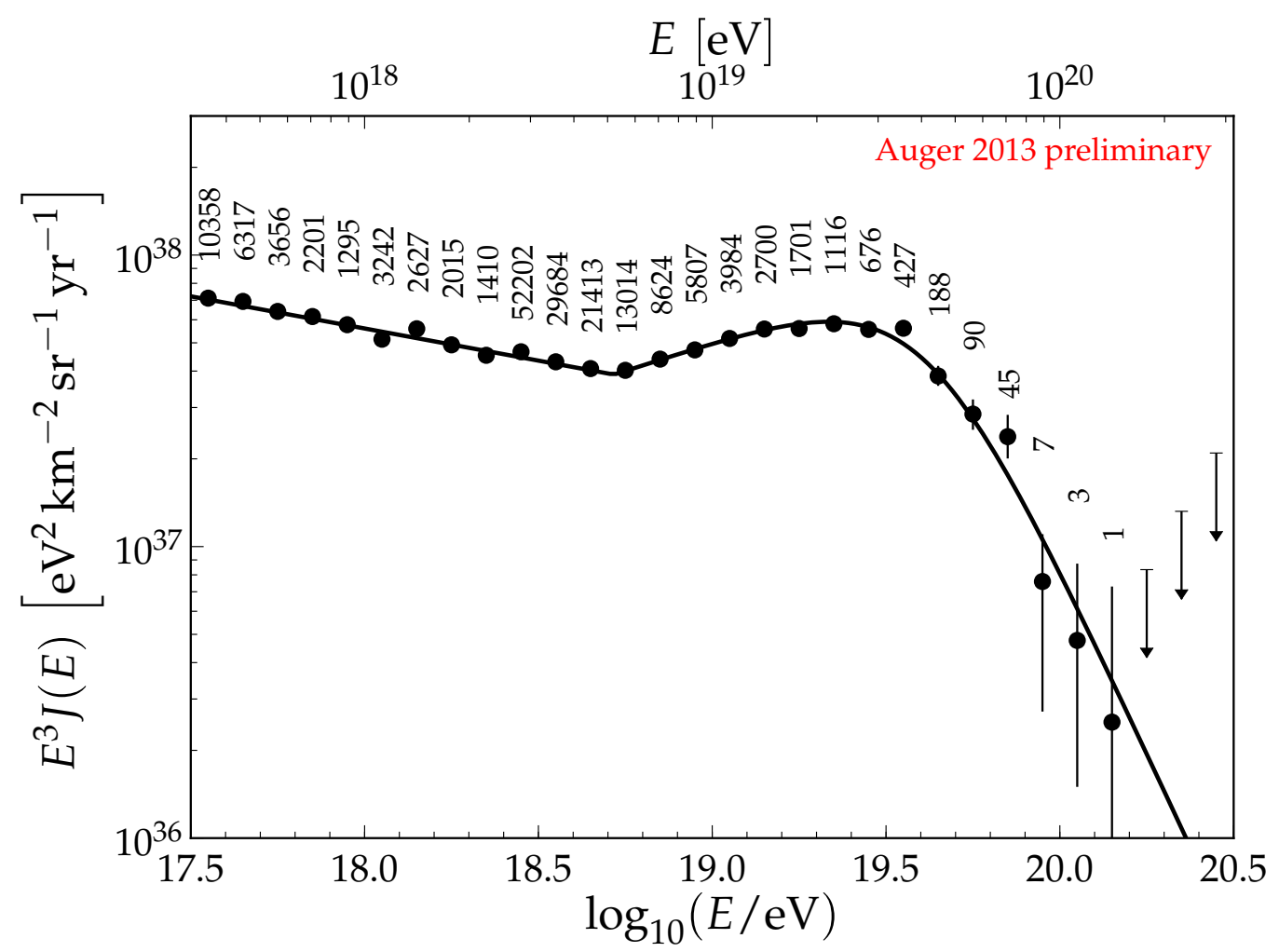

Figura 2.7 - Espectro de energia medido pelo Observatório Pierre Auger (5). Fonte: Figura produzida pela Colaboração Pierre Auger disponível apenas para os membros.

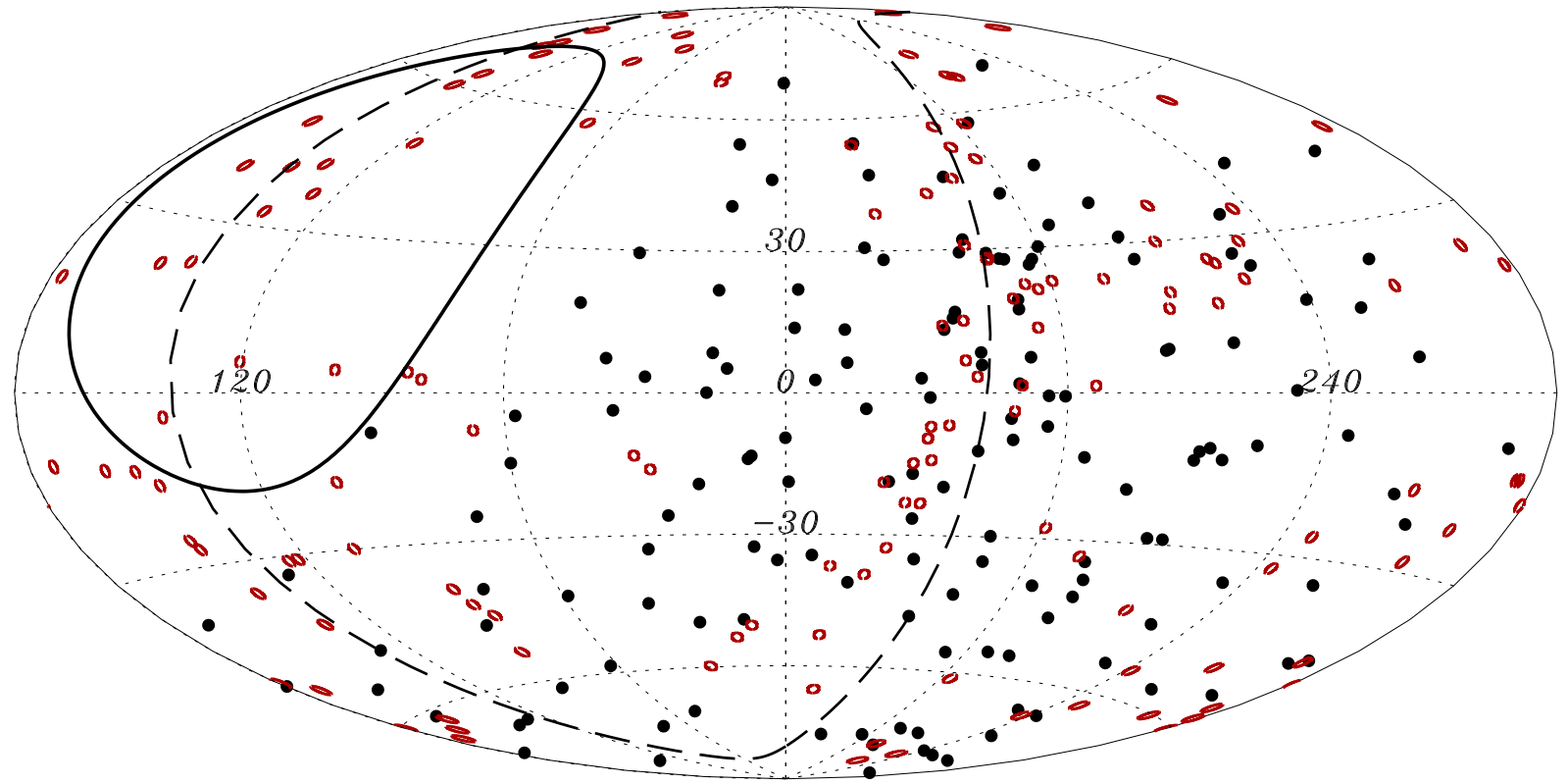

Figura 2.8 - Mapa da direção de chegada dos eventos (pontos pretos) medidos pelo Observatório Pierre Auger com energia superior a $58 \mathrm{EeV}$ em comparação com a posição de AGNs no catálogo Swift-BAT (círculos vermelhos - janela de $1^{\circ}$ ) com distância máxima de $80 \mathrm{Mpc}$. O mapa foi feito em coordenadas galáticas e projeção Aitoff-Hammer. Fonte: Figura produzida pela Colaboração Pierre Auger disponível apenas para os membros. 


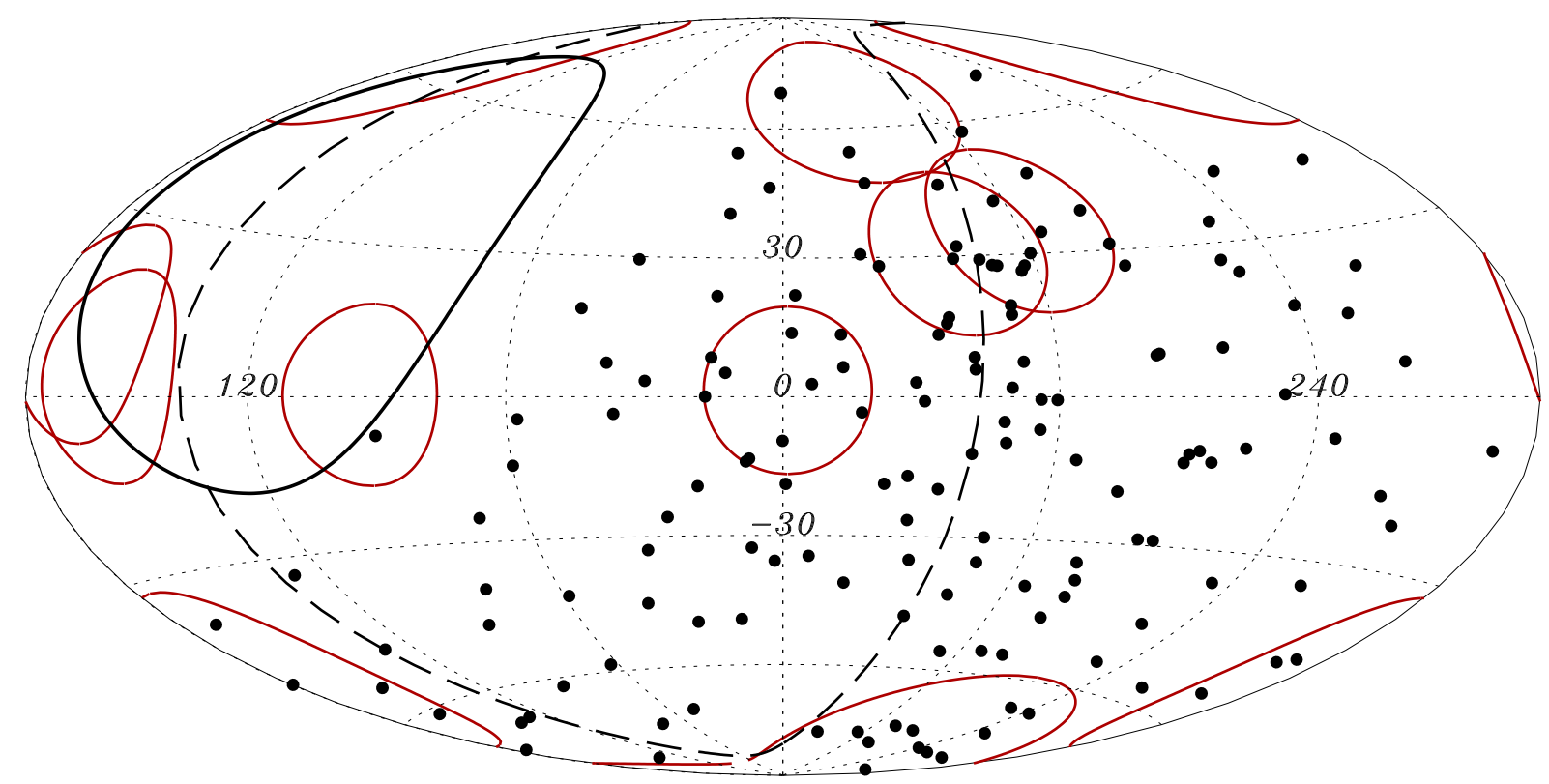

Figura 2.9 - Mapa da direção de chegada dos eventos (pontos pretos) medidos pelo Observatório Pierre Auger com energia superior a $58 \mathrm{EeV}$ em comparação com a posição de AGNs no catálogo Swift-BAT (círculos vermelhos - janela de $18^{\circ}$ ) com distância máxima de $130 \mathrm{Mpc}$ e luminosidade máxima de $10^{44} \mathrm{erg} \mathrm{s}^{-1}$. O mapa foi feito em coordenadas galáticas e projeção Aitoff-Hammer. Fonte: Figura produzida pela Colaboração Pierre Auger disponível apenas para os membros.

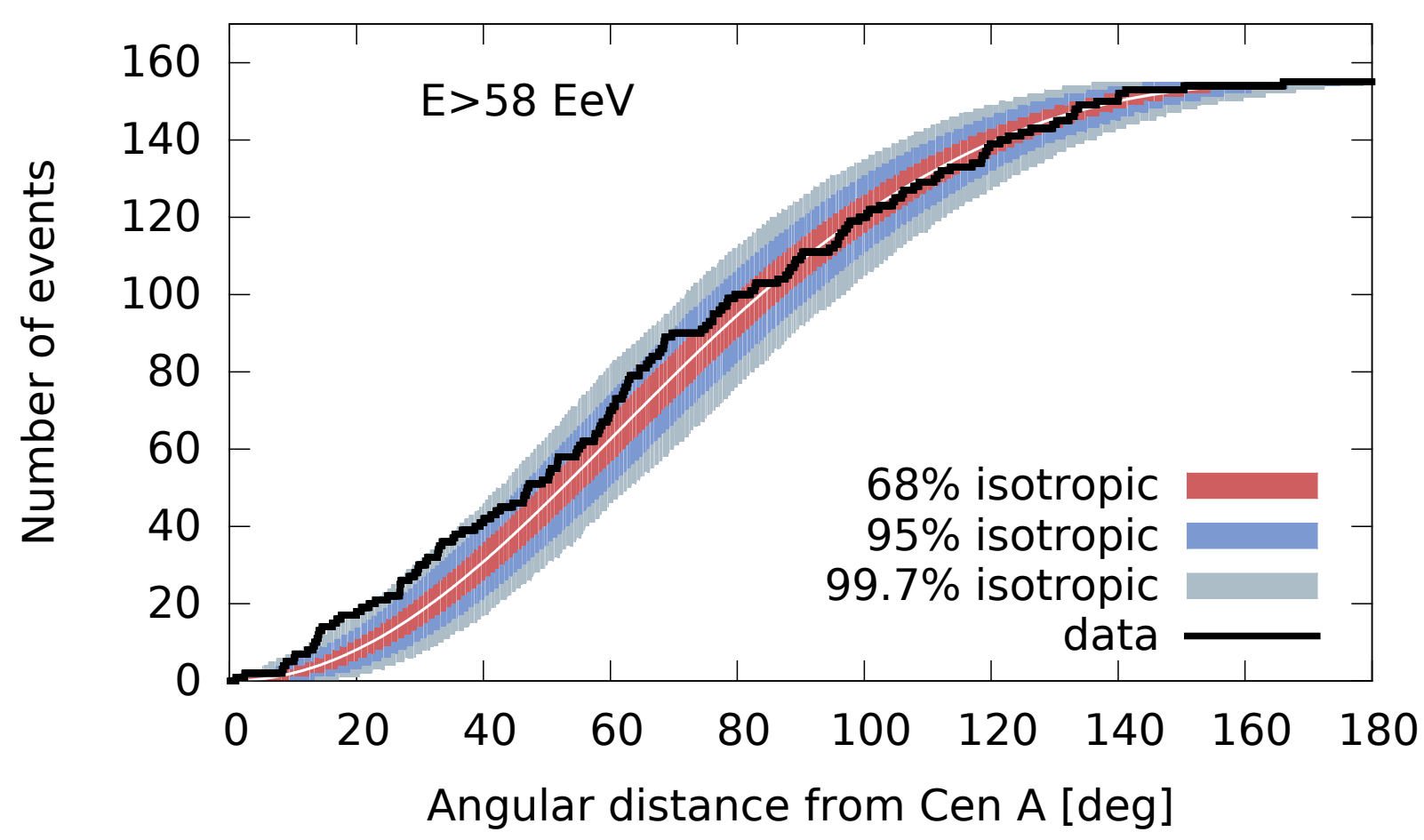

Figura 2.10 - Distribuição do número de eventos em função da distância angular de Centaurus A em comparação com a predição de isotropia. Dados medidos pelo Observatório Pierre Auger (6). Fonte: Figura produzida pela Colaboração Pierre Auger disponível apenas para os membros. 

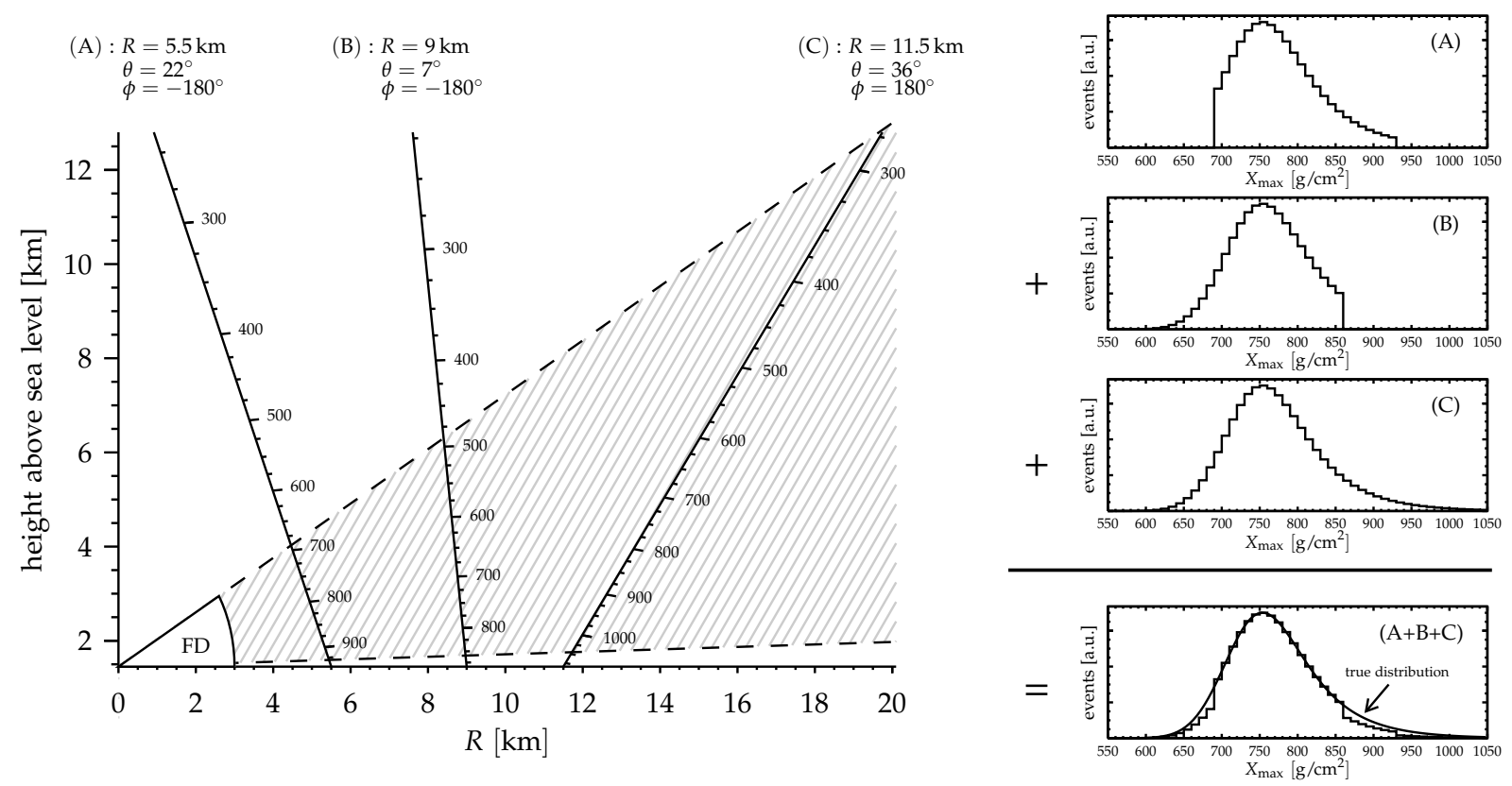

Figura 2.11 - llustração do enviesamento da distribuição de $X_{\max }$ devido à seletividade do campo de visão dos telescópios de fluorescência. O painel da esquerda mostra o campo de visão dos telescópios de fluorescência (linhas tracejadas) sendo cortado por geometrias representativas de chuveiros ( $A, B$ e $C$ ). $O$ painel da direita mostra as distribuições de $X_{\max }$ correspondentes às geometrias. Fonte: Figura produzida pela Colaboração Pierre Auger disponível apenas para os membros.

\subsubsection{Composição e espectro de massas}

A medida mais confiável da composição química é obtida da observação da profundidade do máximo desenvolvimento do chuveiro $\left(X_{\max }\right)$. No entanto, a aceitação do telescópio de fluorescência e a eficiência das técnicas de reconstrução dependem do valor de $X_{\max }$ e uma análise de dados cuidadosa faz-se necessária para garantir a seleção de um conjunto eventos livres de vieses de detecção e distorções de reconstrução.

A figura 2.11 ilustra o efeito seletivo do campo de visão dos telescópios de fluorescência. As linhas tracejadas representam os limites do campo de visão de um telescópio de fluorescência (FD) de forma que a região hachurada é o volume geométrico de detecção. Os eixos $A, B$ e $\mathrm{C}$ representam chuveiros incidentes com diferentes geometrias. As escalas dos eixos mostram a profundidade atmosférica correspondente àquela geometria. Note que os valores de $X_{\max }$ dentro do volume geométrico de detecção não são os mesmos para diferentes geometrias dos chuveiros. Os pontos de intersecção dos eixos com o limite do campo de visão do telescópio determinam os extremos superior e inferior do campo de visão para àquela geometria. A consequência deste efeito seletivo de geometrias está ilustrada nas distribuições de $X_{\max }$ no lado direito da figura. Geometrias do tipo A resultam em cortes de valores baixos de $X_{\max }$, 
geometrias do tipo B resultam em cortes de valores altos de $X_{\max }$ e geometrias do tipo C possibilitam uma total amostragem das distribuições de $X_{\max }$.

Além deste efeito de seleção da geometria do chuveiro, outros efeitos semelhantes e mais sutis são introduzidos pela reconstrução do chuveiro atmosférico e pela análise dos eventos. Dois métodos distintos foram elaborados para lidar com esse problema de forma a garantir a fidedignidade dos dados. O primeiro método baseia-se inteiramente nos dados medidos para estudar as geometrias fiduciais do telescópio e optimizar filtros seletores. Esta técnica ficou conhecida na Colaboração Auger como cortes fiduciais. O segundo método baseia-se inteiramente na simulação dos detectores para estudar os efeitos de seleção e distorção para posteriormente corrigir os dados medidos. Esta técnica costuma ser denominada de deconvolução. Cada técnica tem vantagens e desvantagem que discutiremos brevemente abaixo.

A técnicas de cortes fiduciais são baseadas no estudo ilustrado na figura 2.12. A figura mostra a evolução da média da distribuição de $X_{\max }\left(\left\langle X_{\max }\right\rangle\right)$ em função dos extremos do limite do campo de visão (Xlow e Xup) para àquela geometria. Espera-se que o valor da média não varie em função dos extremos superiores e inferiores do campo de visão a não ser para às geometrias nas quais a distribuição de $X_{\max }$ está sendo cortada por efeitos de seleção do telescópio de fluorescência. As linhas tracejadas delimitam a região de extremos superiores e inferiores do campo de visão dentro da qual o telescópio de fluorescência impõem cortes na medida da distribuição de $X_{\max }$ enviesando a determinação da média. O método de análise denominado cortes de fidelidade baseia-se na exclusão de todos os eventos com geometrias

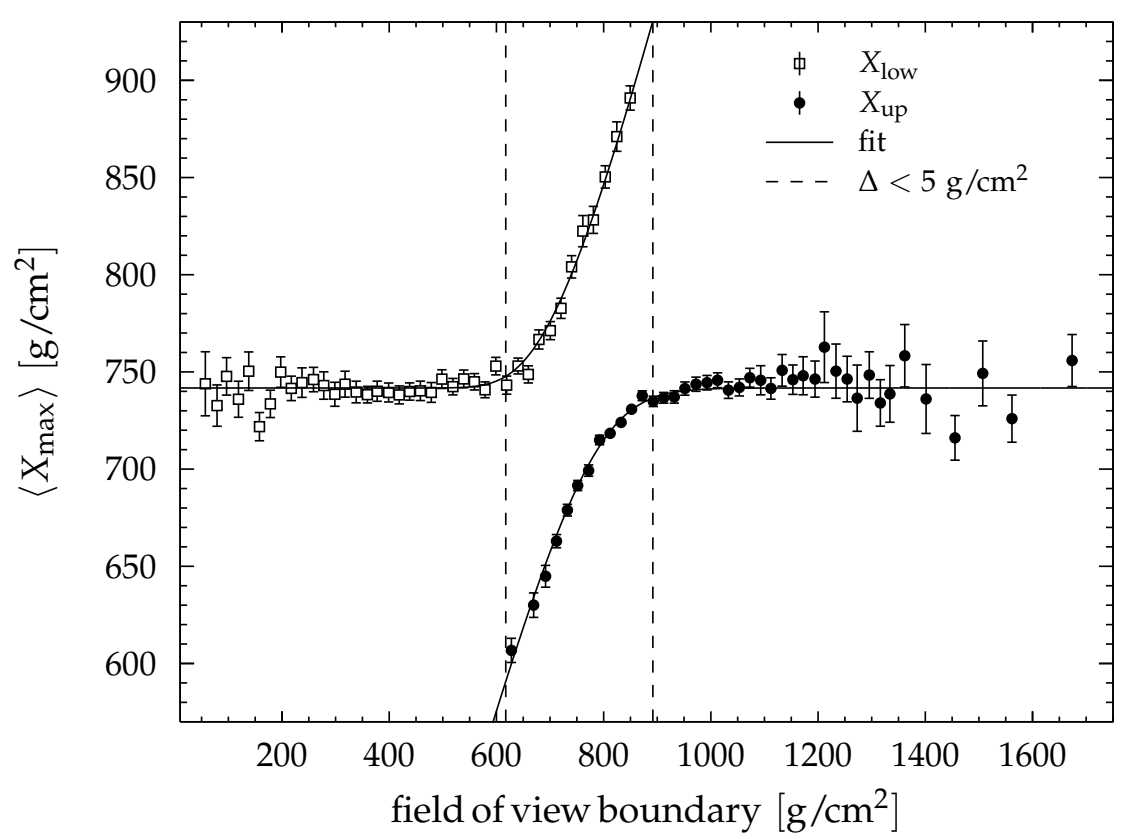

Figura 2.12 - Evolução dos extremos do campo de visão (Xlow e Xup) em função de $X_{\max }$. Fonte: Figura produzida pela Colaboração Pierre Auger disponível apenas para os membros. 
que correspondam à extremos de campo de visão dentro da região delimitada pelas linhas tracejadas. Estudos idênticos foram realizadas para todo o intervalo de energia determinando assim a região fiducial do espaço paramétrico a ser utilizada para as análises de composição.

A consequência imediata deste método é a seleção de um conjunto muito limpo de dados para as análises de composição garantindo um resultado muito confiável e isento de vieses de detecção. Como efeito colateral ele implica numa severa redução do número de eventos disponíveis.

O segundo método de análise baseado em simulações do detector busca corrigir os efeitos de seleção e distorção sem eliminar um número exagerado de eventos. Duas técnicas matemáticas de deconvolução foram implementadas para resolver este problema (8): decomposição em valores singulares (SVD ${ }^{\ddagger}$ ) (73) e interativo Bayesiano (74). O problema pode ser formalmente descrito por uma equação integral:

$$
G(y)=\int A(y, x) F(x) d x
$$

em que $G(y)$ é a função da variável medida, $F(x)$ é a função da variável real e $A(y, x)$ é a função resposta do detector. A equação diferencial acima é chamada de equação de Fredholm de primeiro tipo e é um exemplo de um problema de inversão (75).

No problema da medida de $X_{\max }$, o problema deve ser tratado de maneira discreta, e portanto a equação integral acima se torna um sistema linear de equações que escrevemos na forma matricial como,

$$
\vec{y}=\hat{A} \cdot \vec{x},
$$

em que $\vec{y}$ é o vetor que representa a distribuição medida, $\vec{x}$ é o vetor que representa a distribuição real e $\hat{A}$ é a função resposta do detector em sua forma matricial, também chamada de matriz resposta do detector. No contexto dos métodos de deconvolução, a matriz resposta pode ser interpretada como uma matriz de probabilidade, na qual cada entrada $\hat{A}_{i j}$ representa a probabilidade de medirmos uma grandeza física com valor $y_{j}$ sendo que o valor real é $x_{i}$.

A matriz resposta da medida de $X_{\max }$ dos telescópios de fluorescência foi construída com alto grau de precisão. Simulamos os chuveiros atmosféricos com o programa CONEX (76). Os telescópios de fluorescência podem ser detalhadamente descritos tal como mostrado na referência (77) com os softwares que desenvolvemos na última década. Os detalhes da implementação matemático-computacional podem ser encontrados na referência (8).

\footnotetext{
$\ddagger$ Single Value Decovolution
} 

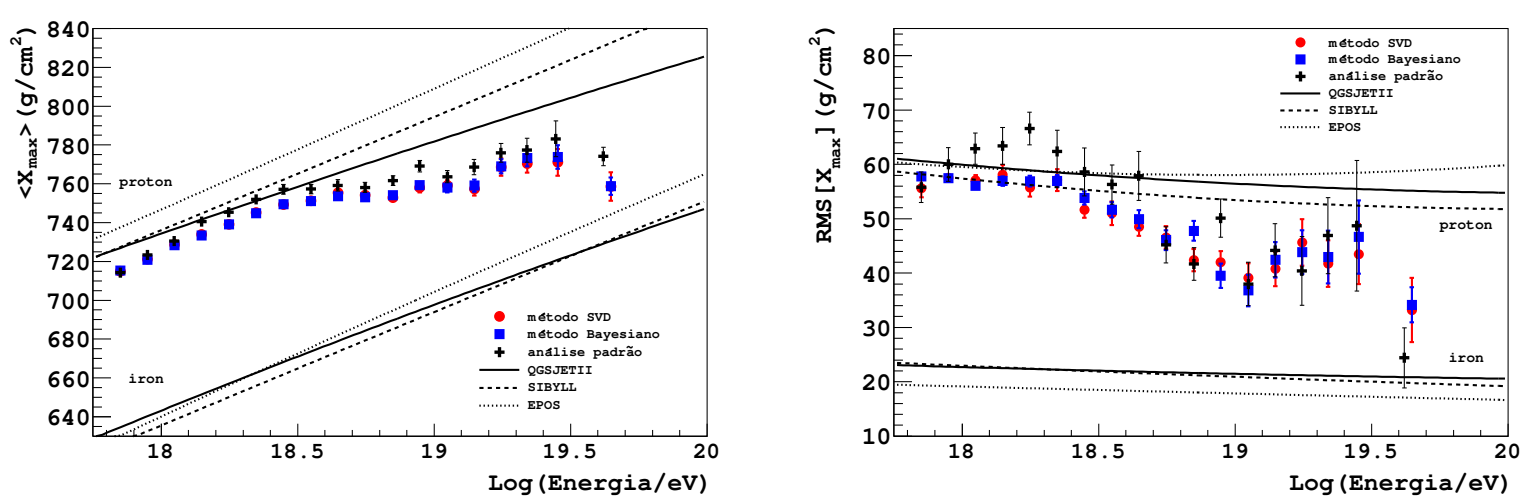

Figura 2.13 - Evolução do logaritmo da massa da partícula primária $(\ln (A))$ em função da energia. Comparação entre as técnicas de remoção de vieses e distorções experimentais. Medidas realizadas pelo Observatório Pierre Auger (7). A legenda "análise padrão" refere-se aos cortes fiduciais. Fonte: Figura extraída da referência (8).

A figura 2.13 mostra a evolução de $\left\langle X_{\max }\right\rangle$ e $\operatorname{RMS}\left(X_{\max }\right)$ com a energia comparando os resultados das técnicas de deconvolução e de cortes fiduciais. A concordância é muito boa.

A figura 2.14 mostra a distribuição de $X_{\max }$ após aplicadas as técnicas de remoção de vieses e distorções experimentais. Todas as técnicas implementadas levam a distribuições indiscrimináveis entre si. A figura 2.15 mostra a evolução dos momentos centrais (média: $\left\langle X_{\max }\right\rangle$ e RMS:RMS $\left.\left(X_{\max }\right)\right)$ da distribuição de $X_{\max }$ em função da energia após completa avaliação de erros sistemáticos e estatísticos. A figura também mostra a predição para próton e núcleos de ferro primários para diferentes modelos de interação hadrônica de altas energias. Um ajuste de duas retas com ponto de intersecção variável descreve muito bem $\left(\chi^{2} / n d f=8.2 / 14\right)$ a evolução da média com a energia e revela uma quebra na tendência da composição em $\log (E / e V)=18,27$.

A interpretação das medidas de $X_{\max }$ só são possíveis quando comparadas as predições das simulações mostradas na figura 2.15. No entanto, os modelos de interação hadrônica utilizados nas simulações para energias acima das disponíveis nos feixes de aceleradores de partículas ( $\mathrm{LHC} \Rightarrow E \sim 10^{17} \mathrm{eV}$ ), são extrapolações de teorias sem confirmação experimental. Se os modelos utilizados estiverem errados, as medidas de $X_{\max }$ teriam outra interpretação no que se refere a composição das partículas primárias.

Os momentos centrais da distribuição de $X_{\max }$ também podem ser transformados no logaritmo da massa $(\ln (A))$ da partícula primária (78) utilizando as simulações dos chuveiros. A figura 2.16 mostra a evolução $\ln (A)$ com a energia para três modelos de interação hadrônica.

As distribuições de $X_{\max }$ foram ajustadas utilizando predições de simulação para quatro (Fe, N, He e p), três (Fe, N e p) e duas (Fe e p) populações de primários (55). As distribuições 
medidas podem ser bem ajustadas por três ou quatro populações entretanto o ajuste com duas populações (Fe e p) não resultou em uma boa descrição dos dados. A figura 2.17 mostra um exemplo do ajuste das distribuições de $X_{\max }$ para o intervalo de energia entre $10^{17.8}$ a $10^{17.9} \mathrm{eV}$. Note os valores de p-value em cada quadro. Cada linha da figura mostra o ajuste para um diferente modelo de interação hadrônica utilizado na simulação dos chuveiros. Cada coluna na figura ilustra o ajuste com duas, três e quatro populações. O mesmo ajuste foi feito para todos os intervalos de energia.

A figura 2.18 mostra a evolução da fração de cada primário em função da energia para os três modelos de interação hadrônica considerados. A fração dos primários pode ser combinada com o espectro de energia (figura 2.7) para encontrarmos o espectro de energia de cada tipo de partícula tal qual mostrado na figura 2.19. 

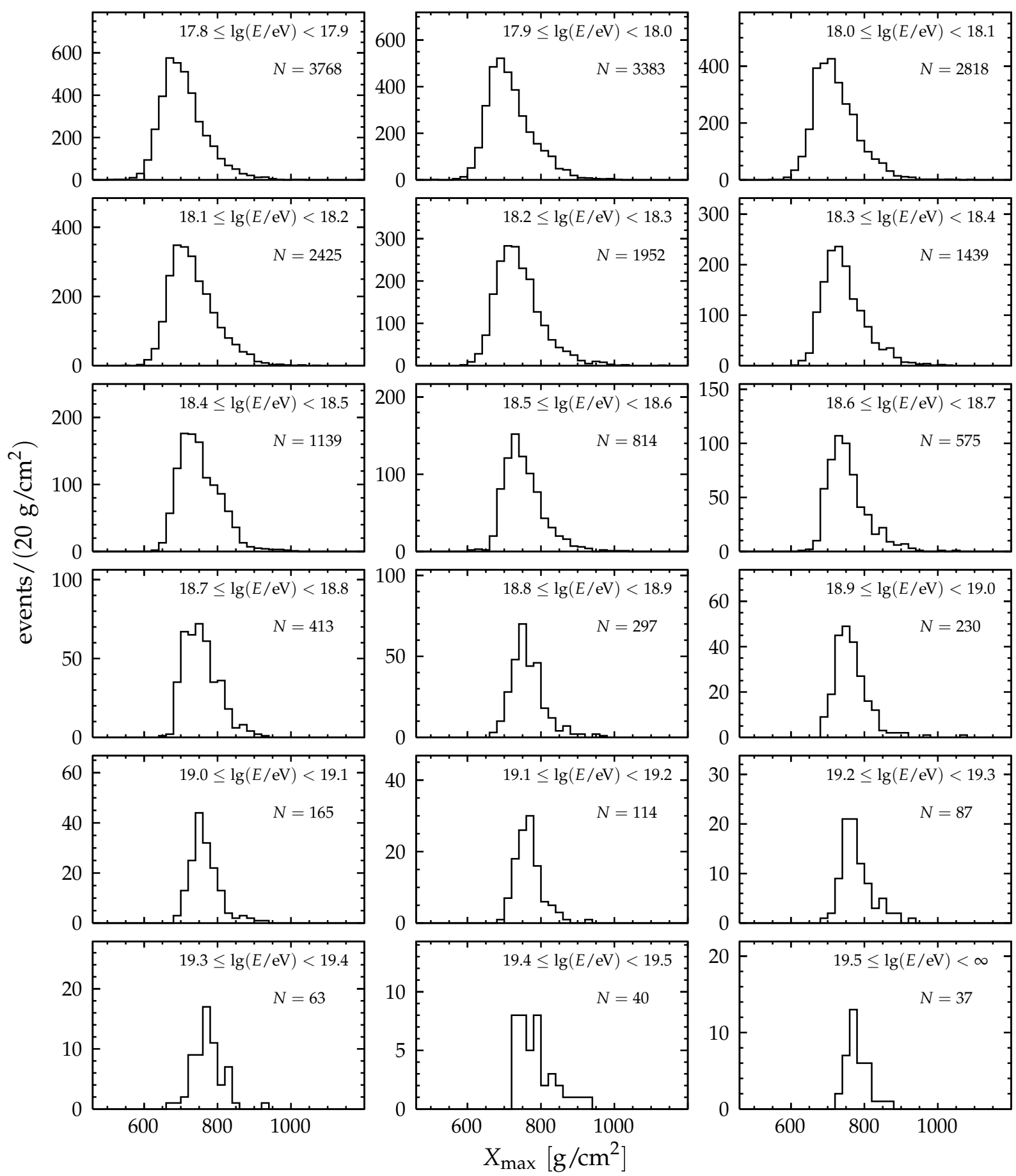

Figura 2.14 - Distribuições de $X_{\max }$ em vários intervalos de energia. Fonte: Figura produzida pela Colaboração Pierre Auger disponível apenas para os membros. 

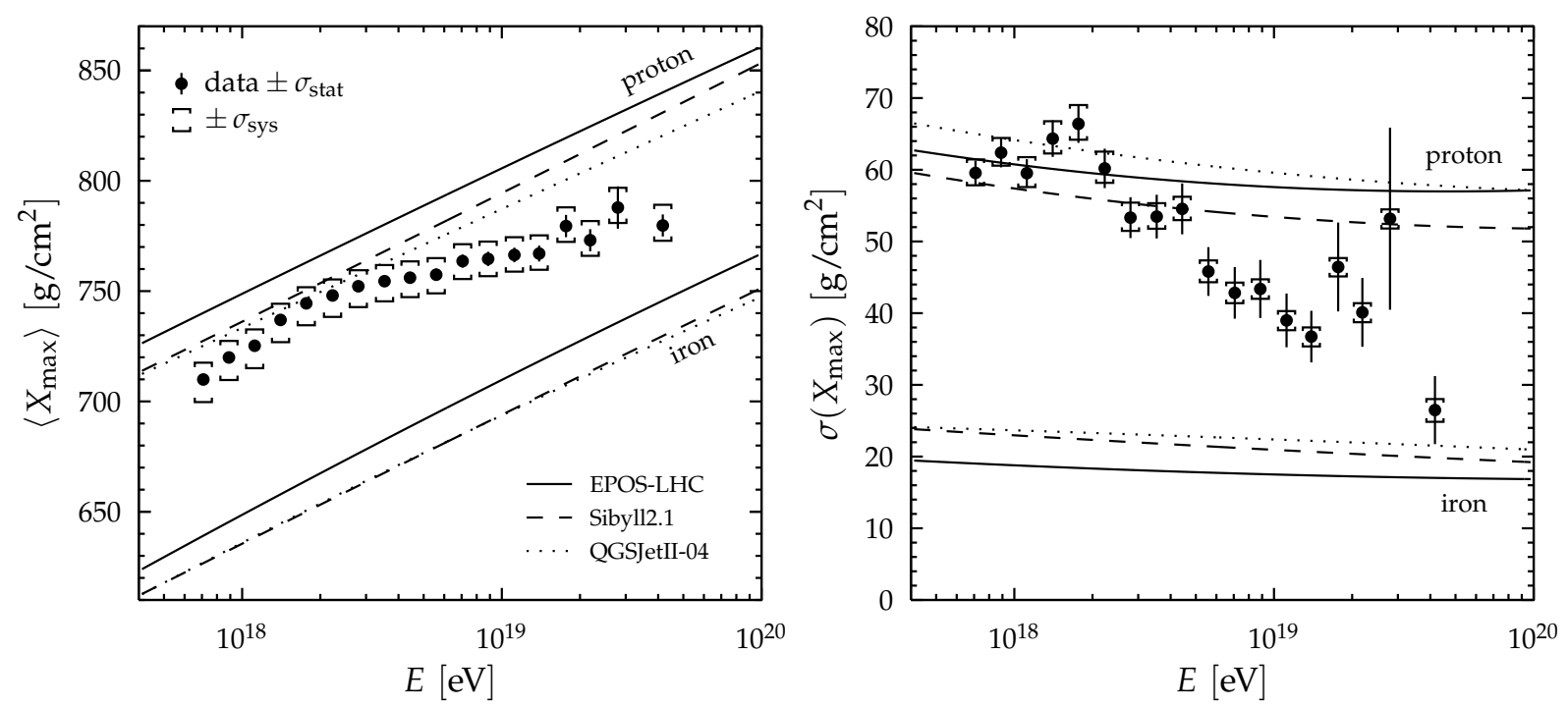

Figura 2.15 - Evolução dos momentos centrais (média e RMS) das distribuições de $X_{\max }$ em função da energia. As linhas mostram predição de simulações para chuveiros iniciados por prótons e núcleos de ferro. Medidas realizadas pelo Observatório Pierre Auger (7). Fonte: Figura produzida pela Colaboração Pierre Auger disponível apenas para os membros.

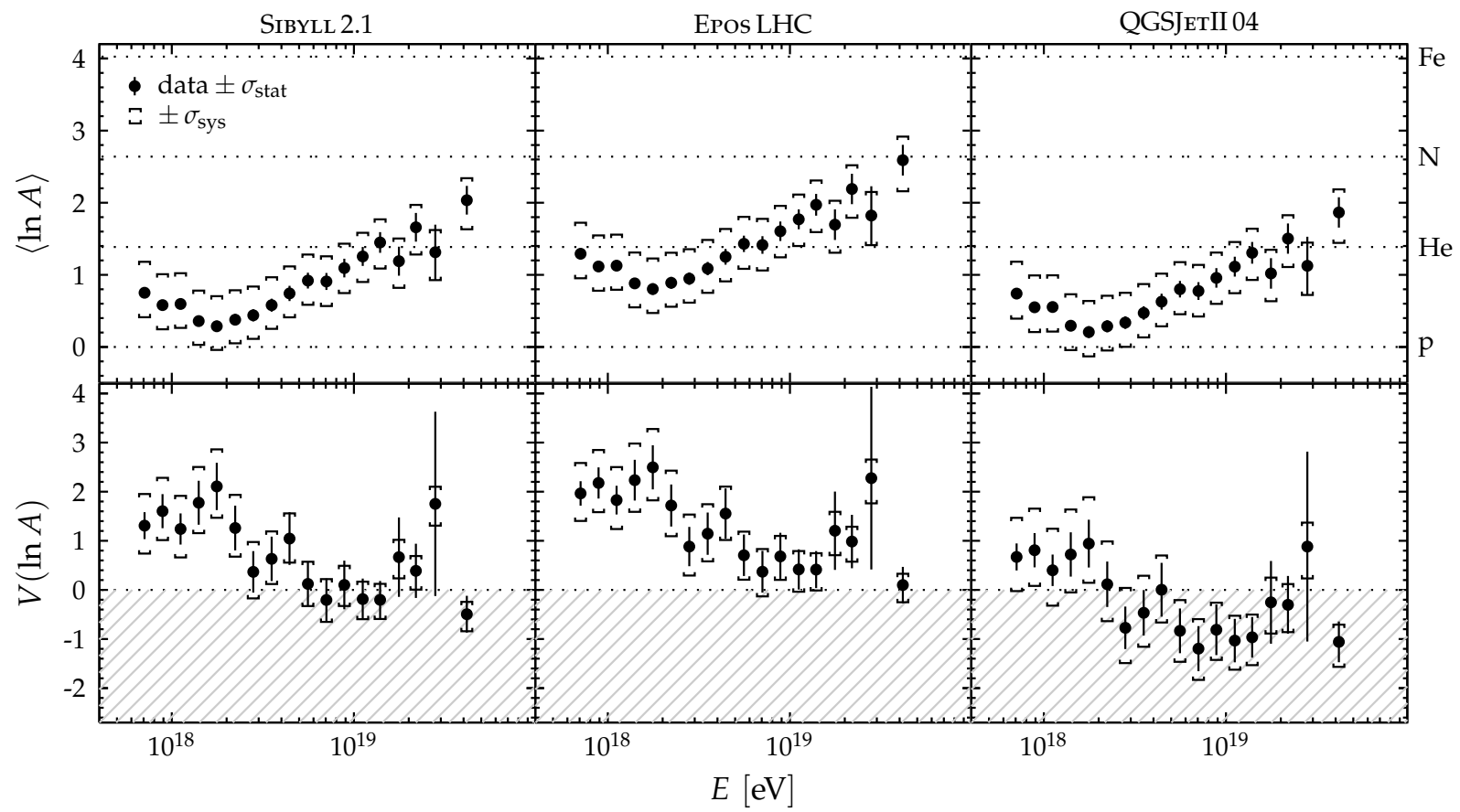

Figura 2.16 - Evolução do logaritmo da massa da partícula primária $(\ln (A))$ em função da energia. Medidas realizadas pelo Observatório Pierre Auger (7). Fonte: Figura produzida pela Colaboração Pierre Auger disponível apenas para os membros. 

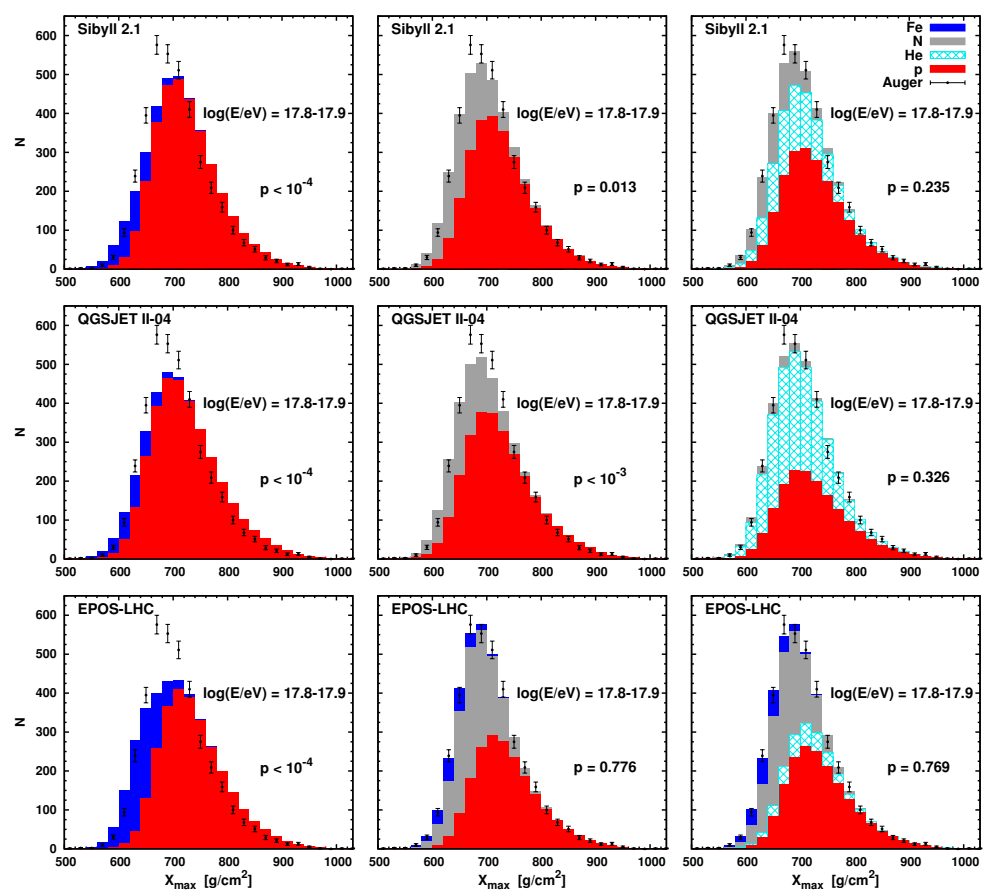

Figura 2.17 - Ajuste das distribuições de $X_{\max }$. Cada linha mostra o ajuste realizado com base em um modelo de interação hadrônica. Cada coluna mostra da esquerda para a direita, o ajuste com dois, três e quatro primários. Note o valor p-value em cada quatro resultante do ajuste. Fonte: Figura produzida pela Colaboração Pierre Auger disponível apenas para os membros.
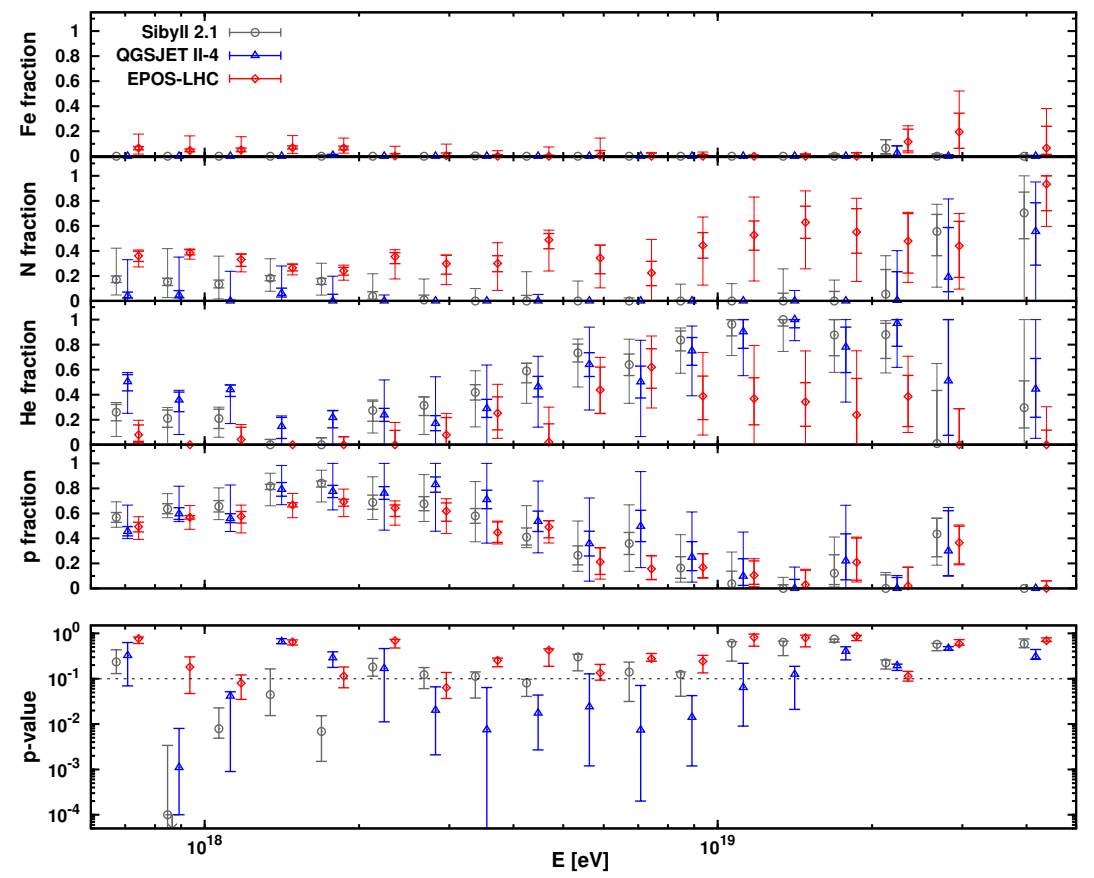

Figura 2.18 - Painel superior: frações de cada tipo de primário ajustadas às distribuições de $X_{\max }$. Painel inferior: Evolução do p-value para cada ajuste com a energia. Fonte: Figura produzida pela Colaboração Pierre Auger disponível apenas para os membros. 


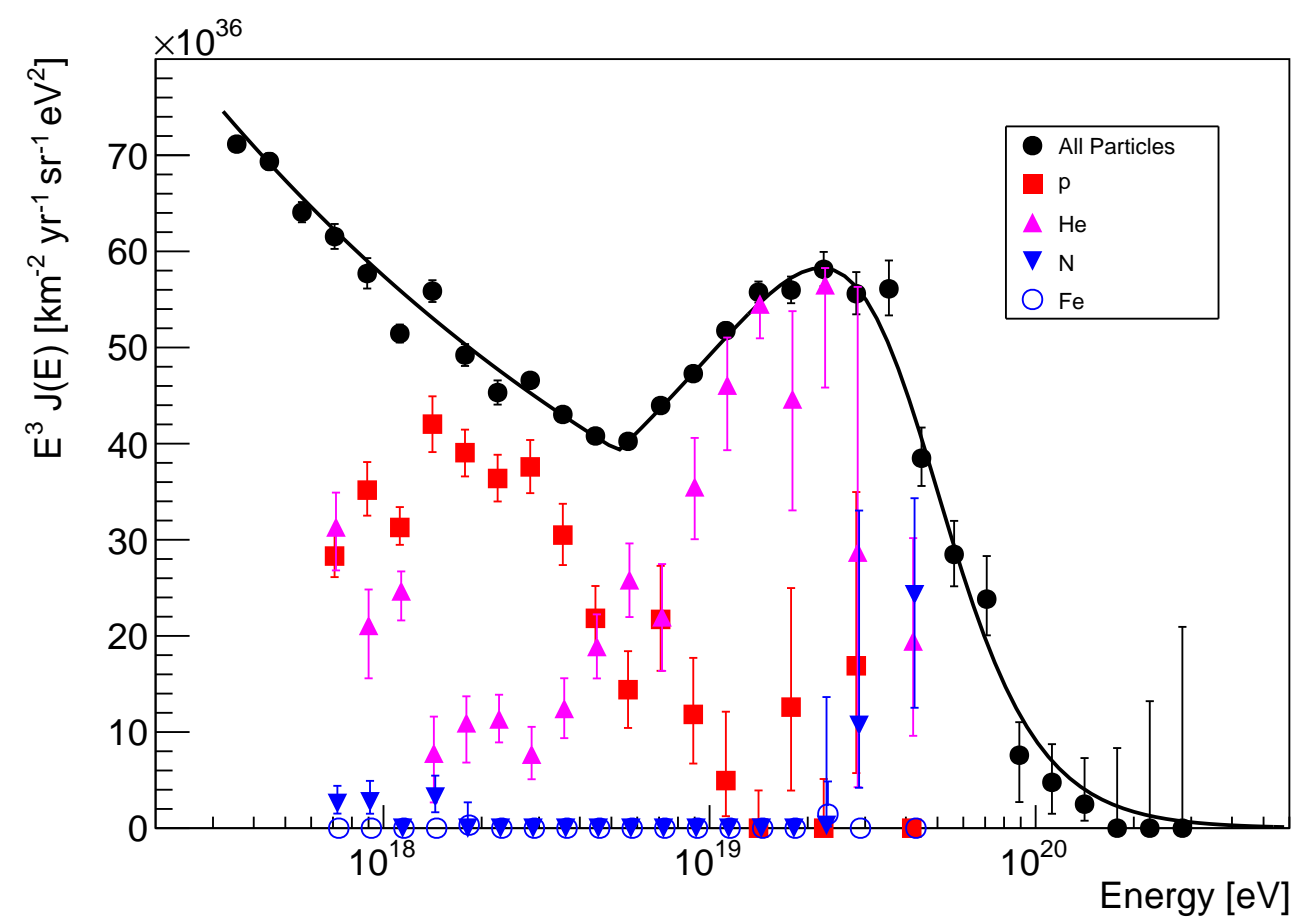

(a) Sibyll 2.1

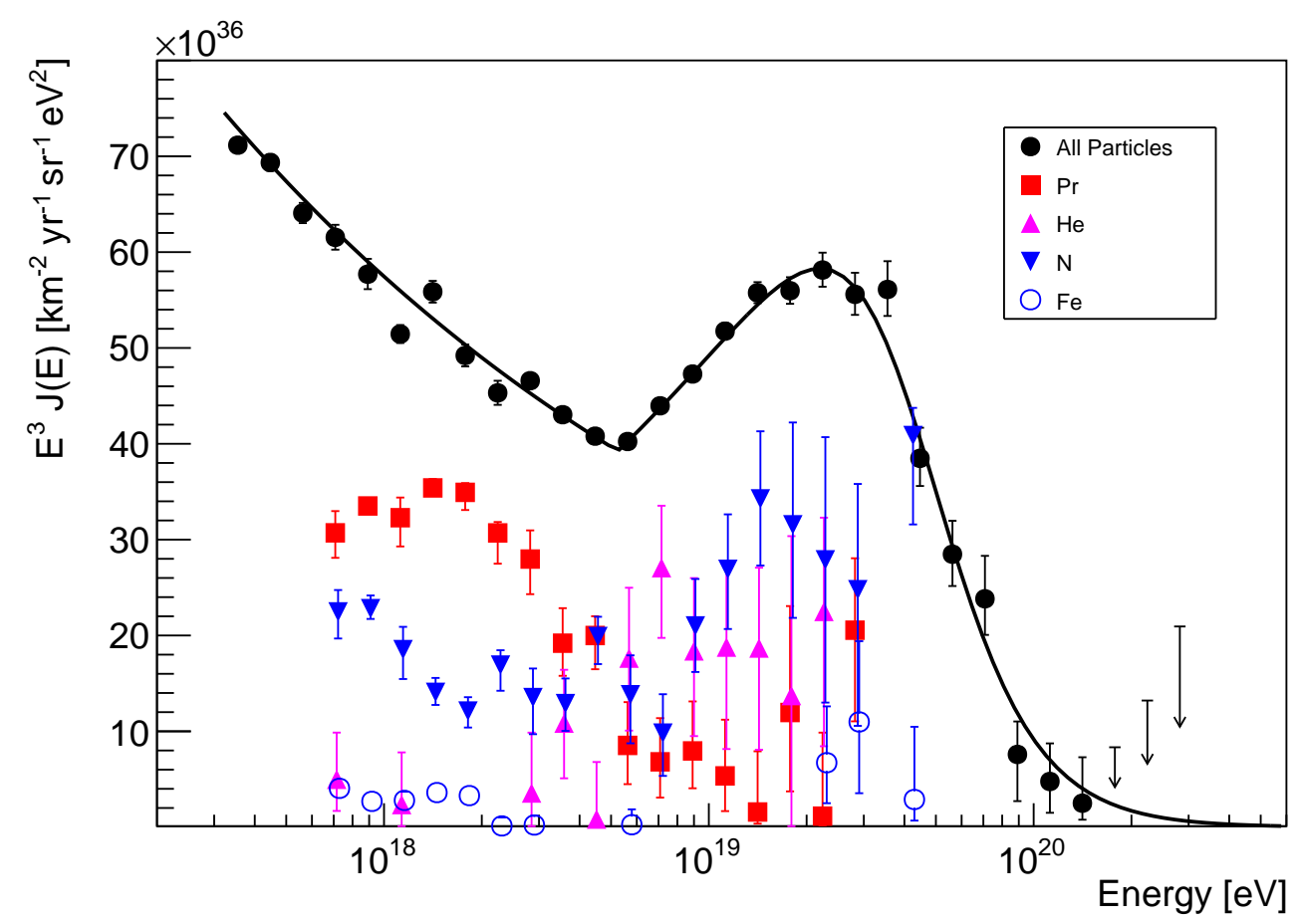

(b) EPOS-LHC

Figura 2.19 - Abundância de cada tipo de primário em função da energia. Fonte: Figura produzida pela Colaboração Pierre Auger disponível apenas para os membros. 


\subsection{Status Experimental}

O Observatório Pierre Auger é um divisor de águas na história do estudo de raios cósmicos de altas energias. A combinação das duas técnicas de medidas, a operação precisa dos detectores por mais de uma década e técnicas de análises inovadoras resultaram na publicação de resultados importantes e inabaláveis. No entanto, várias questões permanecem em aberto apesar dos avanços experimentais.

A ausência de neutrino e fótons com energia superior a $10^{18} \mathrm{eV}$ praticamente exclui todos os modelos de geração de partículas exóticos (79) que tem como característica comum um alto fluxo destas partículas.

A supressão do fluxo acima para energias acima de $\log (E / e V)=19.63$ é condizente com a modulação esperada devido à interação das partículas com a radiação eletromagnética de fundo de micro-ondas $(80,81)$. No entanto, também é compatível com modelos que predizem a exaustão da potência de fontes próximas (47).

A supressão no fluxo coincide, dentro dos erros experimentais, com a energia $(E=55$ $\mathrm{EeV} \Rightarrow \log (E / e V)=19.74)$ no qual a anisotropia das direções de chegada começa a ser estatisticamente significante em estudos de correlação com fontes pontuais. A energia a partir da qual anisotropias de larga escala são detectadas $(\log (E / e V)=18.90)$ coincide dentro do erro experimental com a energia na qual aparece uma quebra no espectro de energia $(\log (E / e V)=18.72)$ conhecida por tornozelo. Por último, a mudança da evolução da composição é vista em mais baixas energias ainda $(\log (E / e V)=18.27)$.

A compatibilidades das medidas fica ainda mais complexa se considerarmos outros experimentos. Infelizmente a proposta de construir um Observatório Pierre Auger Norte não foi aprovada e temos que conviver com uma assimetria entre os dois hemisférios e uma falta de dados no hemisfério norte. No hemisfério norte, o principal experimento em operação é o Telescope Array (TA) que opera telescópios de fluorescência e cintiladores como detectores de superfície. No entanto, a área de detecção do TA é de apenas $730 \mathrm{~km}^{2}$ que é muito pequena quando comparada aos $3000 \mathrm{~km}^{2}$ do Observatório Pierre Auger.

Apesar das diferenças em estatísticas de eventos, tremendos esforços tem sido feitos na tentativa de uma análise conjunta dos dados medidos pelos dois Observatórios: Auger e TA. Buscas por anisotropias de larga escala em mapas completos formados pela junção dos dados medidos por Auger e TA não resultaram na deteç̧ão de dipolos ou multipolos (82). No entanto, a análise dos dados medidos por TA leva a regiões com excesso de eventos bastante 
anisotrópicas (83).

A comparação da evolução de $X_{\max }$ medidos por ambos os experimentos não é trivial dada as diferenças nas análises de dados (84). No entanto, mostramos que os dados medidos por ambos os experimentos são compatíveis dentro das resoluções experimentais.

Podemos resumir em alguns poucos itens as principais medidas à desafiar nossa compreensão dos mecanismos de geração de raios cósmicos de altíssimas energias:

$\log (E / e V)>18.00$ : Ausência de alto fluxo de fótons e neutrinos;

$\log (E / e V)=18.27$ : Mudança na evolução da composição de leve para intermediária ${ }^{\S}$;

$\log (E / e V)=18.72-18.90$ : Tornozelo no fluxo e sinal fraco de anisotropia de larga escala;

$\log (E / e V)=19.63-19.74-19.75$ : Supressão do fluxo, sinal fraco de anisotropia de pequena escala e possível anisotropia norte-sul.

A partir deste quadro, a Colaboração Pierre Auger decidiu melhorar as medidas de composição de raios cósmicos para mais altas energias invadindo às regiões onde as modulações no fluxo e os sinais de anisotropia são detectados. Ao mesmo tempo, pretendemos diminuir a dependência das interpretações da composição em relação aos modelos de interação hadrônico utilizados nas simulações. Isso será possível graças a instalação de novos detectores de múons que permitiram uma medida independente de $X_{\max }$ do tipo da partícula primária. Esta proposta de ampliação das potencialidades iniciais do Observatório Pierre Auger está em fase de estudo e foi nomeada AugerPrime. Mais detalhes serão dados no último capítulo desta tese.

$\S$ Considerando correto as extrapolações propostas pelos modelos de interação hadrônica. 


\section{Propagação de Raios Cósmicos de Altíssimas Energias}

O caminho das partículas cósmicas da fonte até a Terra não é vazio. Campos magnéticos e radiação eletromagnética preenchem o Universo e podem interagir com as partículas cósmicas ao longo do seu trajeto. O efeito das interações podem desviar a trajetória da partícula, causar perda de energia e criar um jato de outras partículas. Assim, as propriedades das partículas medidas na Terra e discutidas no capítulo 2 não são iguais às propriedades das mesmas ao saírem da fonte aceleradora. Desta forma, o estudo dos objetos astrofísicos e dos mecanismos neles operando só é possível graças à descrição das mudanças das propriedades das partículas ao longo do caminho.

Ao mesmo tempo, a interação das partículas com o meio intergaláctico oferece uma oportunidade de estudo das propriedades do meio. Suposições teóricas a respeito da densidade de radiação e da intensidade e estrutura dos campos magnéticos podem ser confrontadas com medidas experimentais do espectro de energia e da direção de chegada das partículas na Terra (34). Nosso conhecimento sobre os campos magnéticos intergalácticos é bastante escasso e raios cósmicos de altas energias representam um possibilidade concreta de estudo das suas características no Universo local (85).

A densidade de energia dos campos magnéticos intergalácticos é suficiente para isotropisar raios cósmicos com energia da ordem de $10^{18} \mathrm{eV}$ (86). Mesmo o campo magnético galático pode representar uma importante mudança da direção de propagação apagando sinais de fontes locais (87). Além de interferir na informação da direção da fonte aceleradora, os campos magnéticos aumentam o caminho total percorrido pela partícula da fonte até a Terra, provocando assim indiretamente, um aumento do efeito da interação das partículas com a radiação de fundo do Universo.

Não discutiremos aqui o efeito dos campos magnéticos na propagação de raios cósmicos e para o leitor interessado sugerimos as referências $(86,88,89)$. Volumosas revisões sobre as interações relevantes das partículas com a radiação de fundo no Universo e seus efeitos moduladores do espectro de energia e da composição química tem sido escritas nos últimos 
anos. Para um estudo completo, recomendamos a consulta das referências $(40,90)$. Não temos o intuito de repetir aqui as discussões destas referências. Faremos apenas uma breve revisão dos principais efeitos e concentraremos nossa atenção em um aspecto da propagação de raios cósmicos de altíssima energia no qual temos trabalhado recentemente. A saber, discutiremos a proposta por nós apresentada de determinação da luminosidade de UHECR de fontes pontuais através da medida do limite superior do fluxo de raios gamas com energia de GeV-TeV.

\subsection{Radiação de fundo no Universo}

O Universo é repleto de radiação produzidas pelos mais variados mecanismos em todos os comprimentos de onda. A figura 3.1 mostra o espectro a radiação de fundo no Universo divido em três componentes com suas respectivas luminosidades. Cada tipo de partícula cósmica (fótons, prótons, núcleos e neutrinos) interage com certas faixas de frequência da radiação de fundo.

A radiação cósmica no óptico $(\lambda \geq 8 \mu \mathrm{m})$ (COB - Cosmic Optical Background) como definida na referência (9) inclui radiação no infravermelho próximo, visível e ultravioleta. Esta definição separa a radiação cósmica no infravermelho $(\lambda<8 \mu \mathrm{m})$ (CIB - Cosmic Infrared Background) no seu subgrupo específico. Outra definição mais abrangente e usualmente encontrada na literatura é de luz extragaláctica de fundo (EBL - Extragalactic Background Light) que inclui radiação no infravermelho, visível e ultravioleta. Note que $E B L=C O B+$ CIB.

A medida da EBL não é trivial pois o sinal é contaminado por radiação produzidas em fontes pontuais e poeira. Assim, a determinação desta componente de fundo só é possível através da modelagem deste ruído ao longo da história do Universo, o que envolve inevitavelmente parâmetros da formação e da evolução de estrelas e galáxias que só são conhecidos com grande incerteza experimental $(31,32)$.

A Cosmic Microwave Background - Radiação Cósmica de Micro-ondas (CMB) é uma fotografia do Universo com aproximadamente 400 mil anos de idade. Neste período, o Universo tornou-se frio o suficiente para permitir a formação de átomos o que liberou o caminho para a radiação se expandir livremente. O Universo ficou transparante para a radiação que o ocupou completamente preservando as minúsculas variações de temperatura deste momento como flutuações na densidade da radiação. 


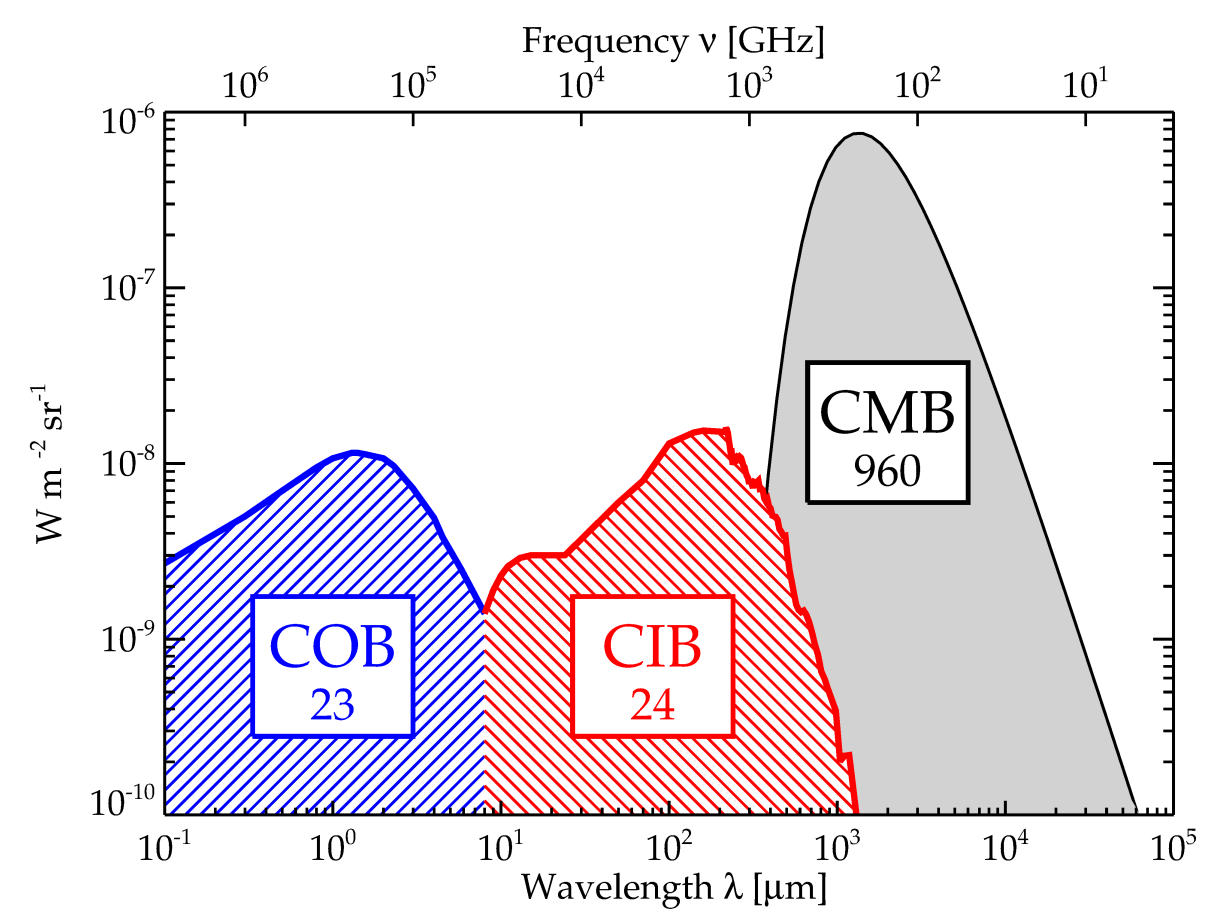

Figura 3.1 - Espectro da radiação de fundo divido em três componentes: COB (óptico), CIB (infravermelho) e CMB (micro-ondas). Fonte: Figura extraída da referência (9).

A radiação de fundo de micro-ondas tem sido medida com grande precisão (91-93) e hoje é a informação mais confiável e valiosa na qual baseiam-se os modelos cosmológicos vigentes. O espectro da CMB é um espectro de corpo negro com temperatura $2.72548 \pm 0.00057$ Kelvin (19). As análises direcionais mostram que a CMB é altamente isotrópica (93).

CMB e CIB deixam marcas indeléveis na propagação de partículas cósmicas carregadas com energia superior à $\mathrm{EeV}$. COB tem um papel menor e praticamente desprezível na propagação de partículas cósmicas carregadas, por outro lado, interfere decisivamente na propagação de fótons com energia superior a $\mathrm{GeV}$.

A referência (10) estudou em detalhes a propagação de fótons com energia superior a $\mathrm{GeV}$. A figura 3.2 resume os resultados deste estudo através do livre caminho médio da interação de fótons de altas energias ( $\mathrm{GeV}$ ) com a radiação de fundo nos seus vários comprimentos de onda. A figura mostra o livre caminho da interação de um fóton com a radiação de fundo em função da energia do fóton. Em verde logo acima da curva, está escrito o comprimento de onda da radiação de fundo que mais contribui na determinação do livre caminho médio. Note na figura que a radiação cósmica em rádio, não mostrada na figura 3.1 e não incluída nas definições de $\mathrm{COB}, \mathrm{CIB}$ e EBL, interfere na propagação de fótons com energia acima de $10 \mathrm{EeV}$.

Preocupados com a propagação de partículas carregadas de altíssima energias, estudamos 


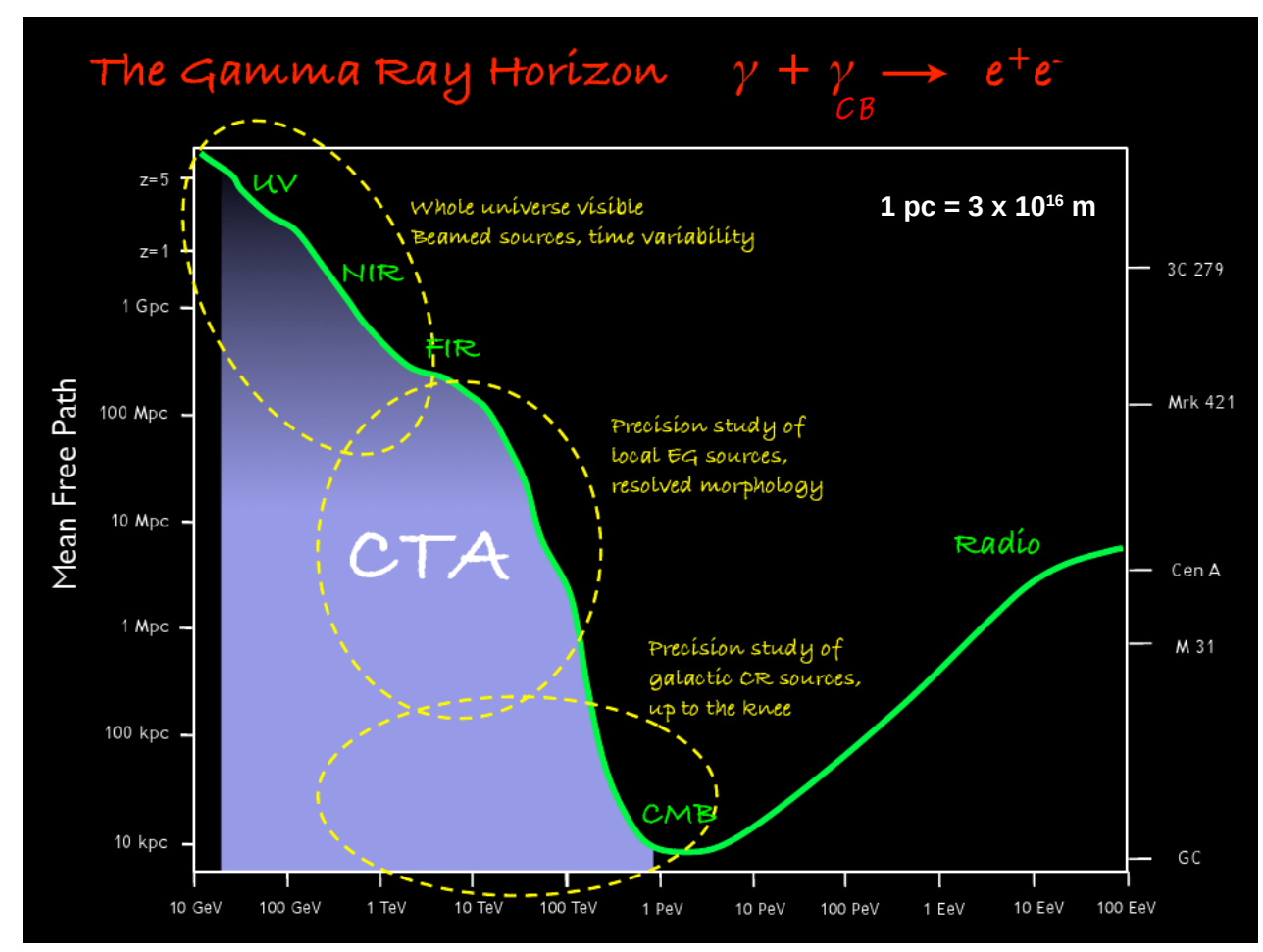

Figura 3.2 - Livre caminho médio de fótons na radiação de fundo em função da energia do fóton. A figura mostra o prospecto de estudo do Observatório CTA. Fonte: Adaptado da referência (10)

em detalhes a CIB (30). A figura 3.3 mostra uma compilação das medidas experimentais. A figura 3.4 mostra os modelos derivados destas medidas utilizando suposições sobre a evolução do ruído. A figura 3.5 mostra os modelos mais utilizadas nos cálculos de propagação de partículas carregadas em comparação com COB. Note que a discrepância entre os modelos de CIB utilizados é significativa e exige esforços para redução das diferenças. 


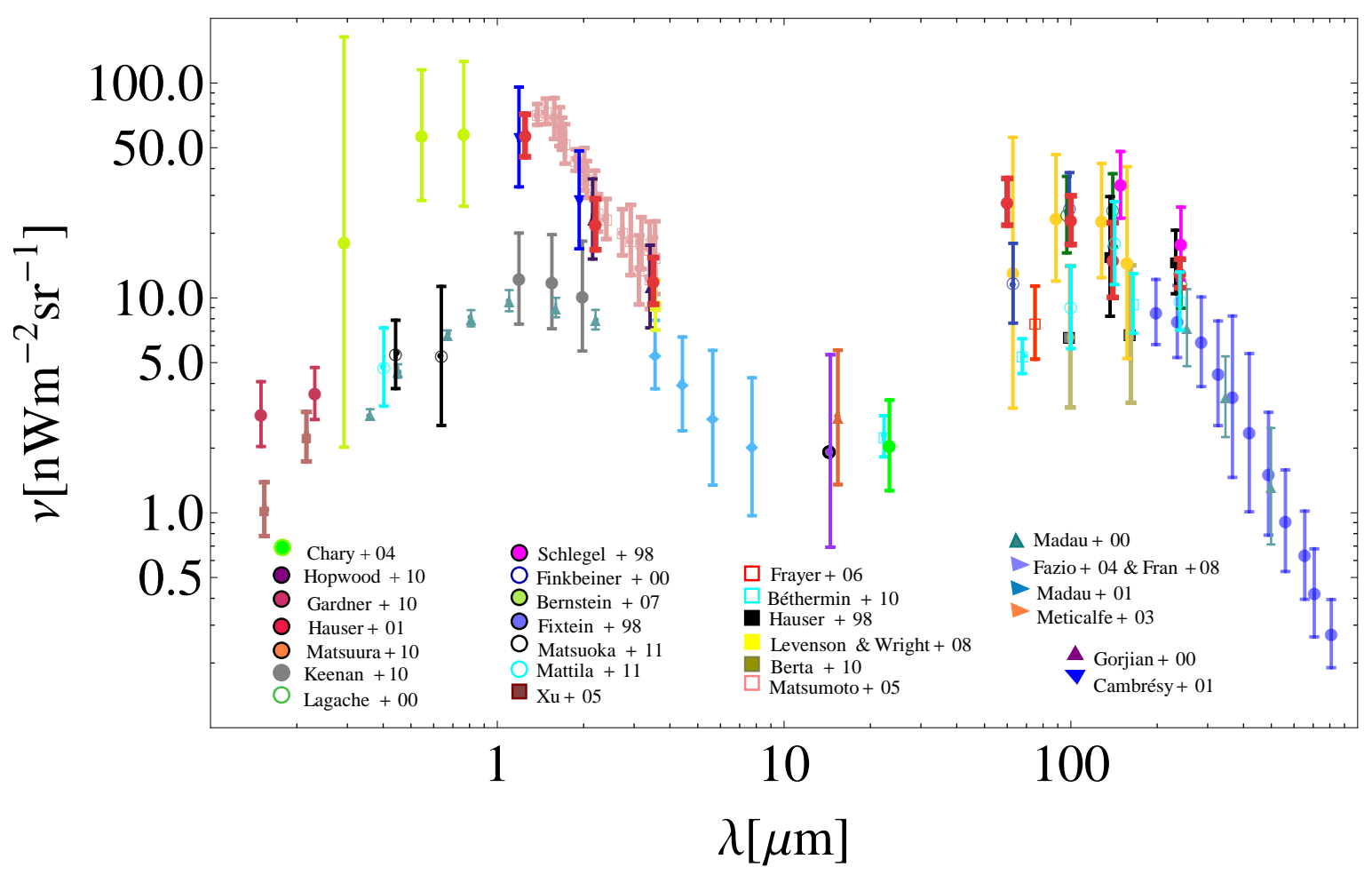

Figura 3.3 - Medidas da radiação infravermelho de fundo. Chary (11), Gardner (12), Hauser+01 (13), Matsuura (14), Keenan (15), Lagache (9), Schlegel (16), Finkbeiner (17), Bernstein (18), Fixsen (19), Xu (20), Frayer (21), Béthermin (22), Hauser+98 (23), Levenson\&Wright (24), Berta (25), Madau (26), Fazio\&Fran (27), Madau (26), Metcalfe (28), Gorgin (11), Cambrésy (29). Fonte: Figura extraída da referência (30). 


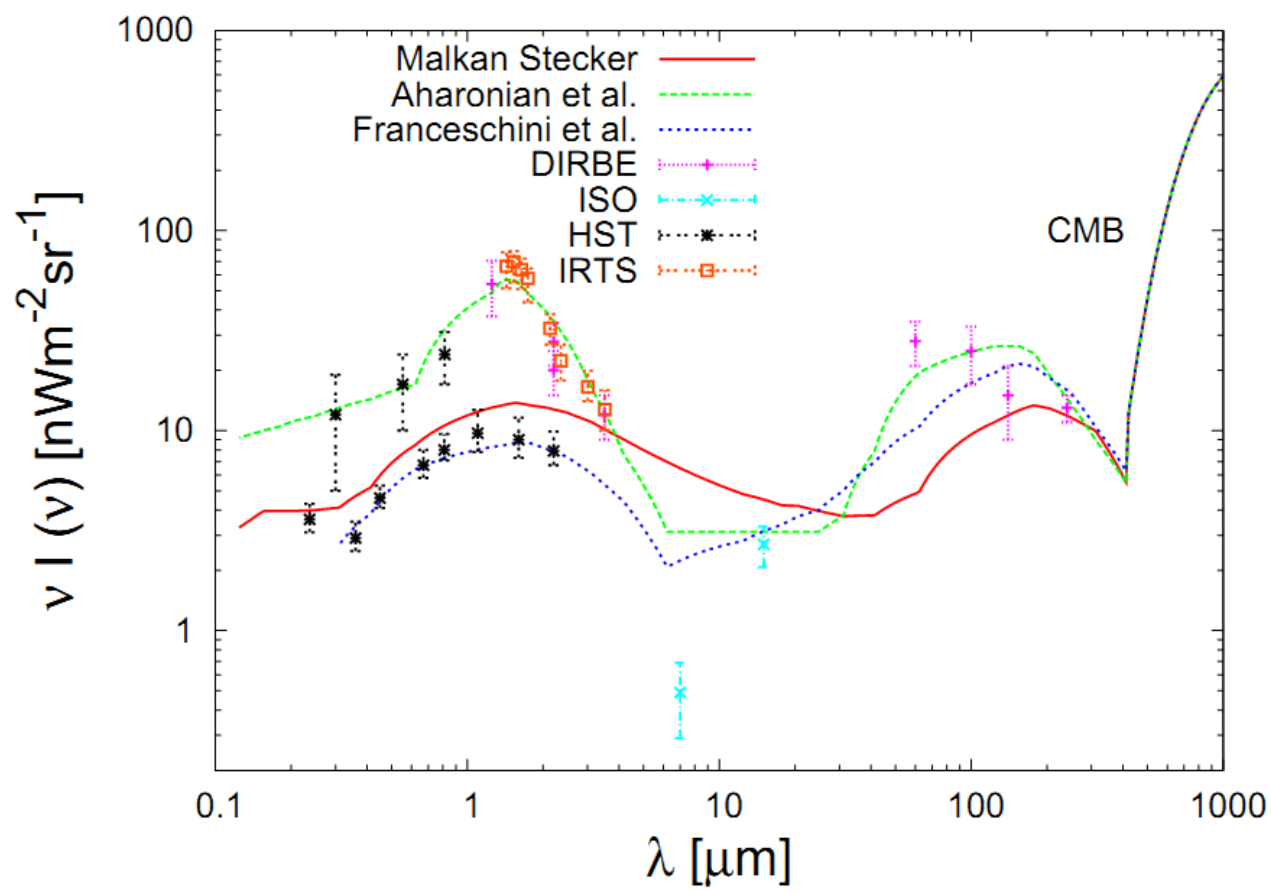

Figura 3.4 - Modelos da radiação cósmica infravermelha. Malkan\&Stecker (31), Dominguéz (32), Aharonian+03 (33), Mazin\&Raue (34), Albert (35), Aharonian+06 (36), Franceschini+01 (37), Franceschini+08 (38), Gilmore (32) e Kneiske (39). Fonte: Figura extraída da referência (30).

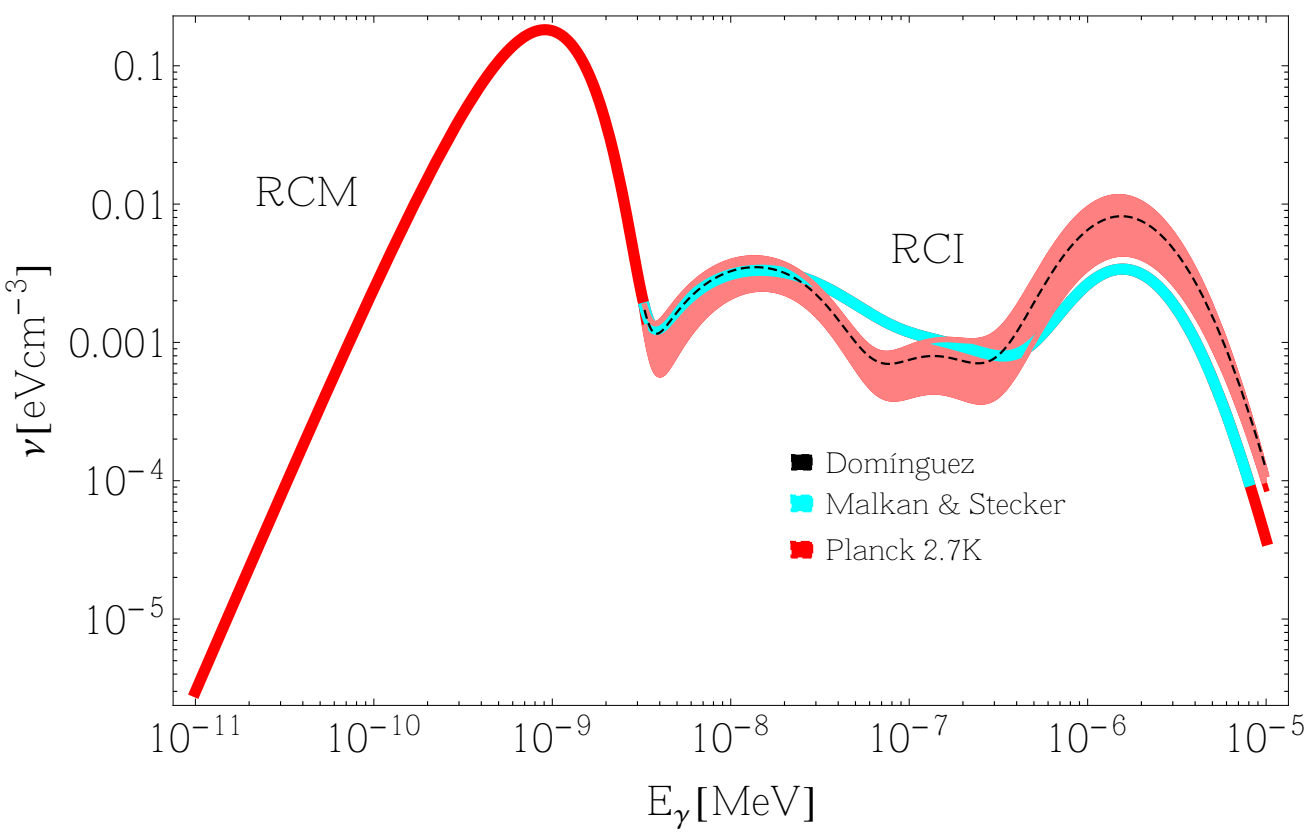

Figura 3.5 - Principais modelos de radiação cósmica infravermelha utilizados na propagação de UHECR. Malkan\&Stecker (31) e Dominguéz (32). Fonte: Figura extraída da referência (30). 


\subsection{Principais interações de UHECR com a radiação de fundo}

As interações de uma partícula cósmica viajando pelo universo varre um espectro abrangente de energia e portanto de interações. No sistema de referência da partícula cósmica (SRPC), um fóton com energia inferior à massa de repouso de um par elétron-pósitron $\left(E_{\gamma}^{S R P C}<1 \mathrm{MeV}\right)$ realiza uma interação eletromagnética clássica com um partícula pontual carregada. Se $E_{\gamma}^{S R P C}>1 \mathrm{MeV}$ a estrutura quântica do elétron se revela e a produção de partículas começa a ser possível nas interações (produção de pares). Se $E_{\gamma}^{S R P C}$ for maior que a energia de ligação dos núcleons em um núcleo $\left(E_{\gamma}^{S R P C} \sim 8 \mathrm{MeV}\right)$ a estrutura nuclear começa a ser revelada e processos de fragmentação começam a ser importantes (fotodesintegração). Se $E_{\gamma}^{S R P C}$ for maior que a escala de energia da massa de repouso dos mésons $\left(E_{\gamma}^{S R P C} \sim 140\right.$ $\mathrm{MeV}$ ) a estrutura hadrônica dos núcleons é ressaltada e processos de produção de píons são predominantes (foto-produção de píons). Se $E_{\gamma}^{S R P C}$ for maior que $1 \mathrm{GeV}$ a estrutura hadrônica do fóton é despertada e processos múltiplos de produção de mésons começão a ser importantes (produção múltipla de píons).

A figura 3.6 mostra a seção de choque da interação de um próton com um fótons em função da energia do fóton no sistema de referência do próton. Os pontos são medidas e as curvas os modelos elaborados na referência (40). As curvas denominadas direct e resonances refere-se ao mecanismo de produção de partículas nas interações.

A figura 3.7 mostra a seção de choque da interação de um núcleo de ferro com um fóton em função da energia do fóton no sistema de referência do núcleo de ferro. As curvas são resultados dos cálculos feitos na referência (40). São mostradas as curvas referentes a cada fenômeno: Giant Dipole Ressonance (GDR), Quasi-Deuteron process (QD), baryon resonance excitation (ISOBAR) e fragmentação do núcleo. 


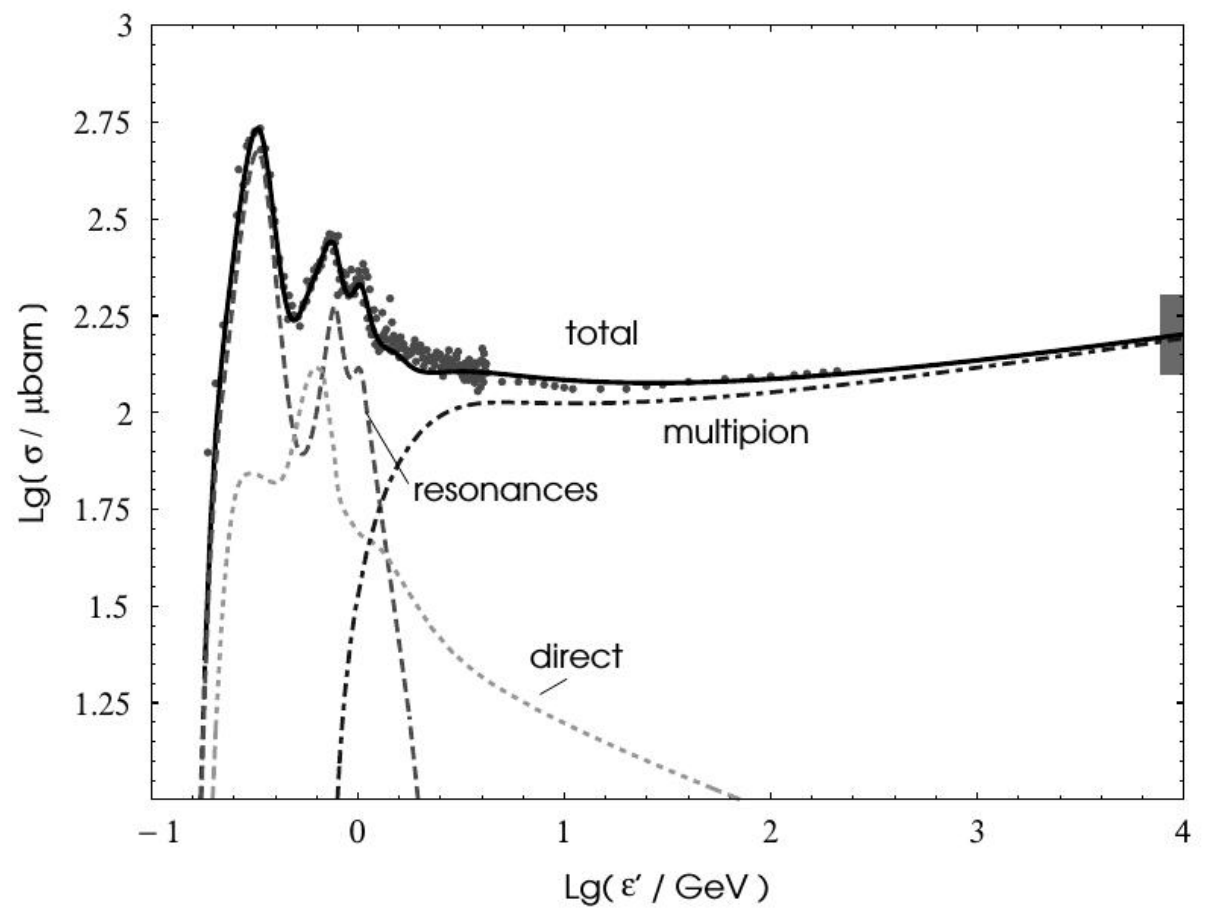

Figura 3.6 - Seção de choque da interação próton-fótons em função da energia do fótons no sistema de referência do próton $E_{\gamma}^{S R P C}=\epsilon^{\prime}$. Fonte: Figura extraída da referência (40).

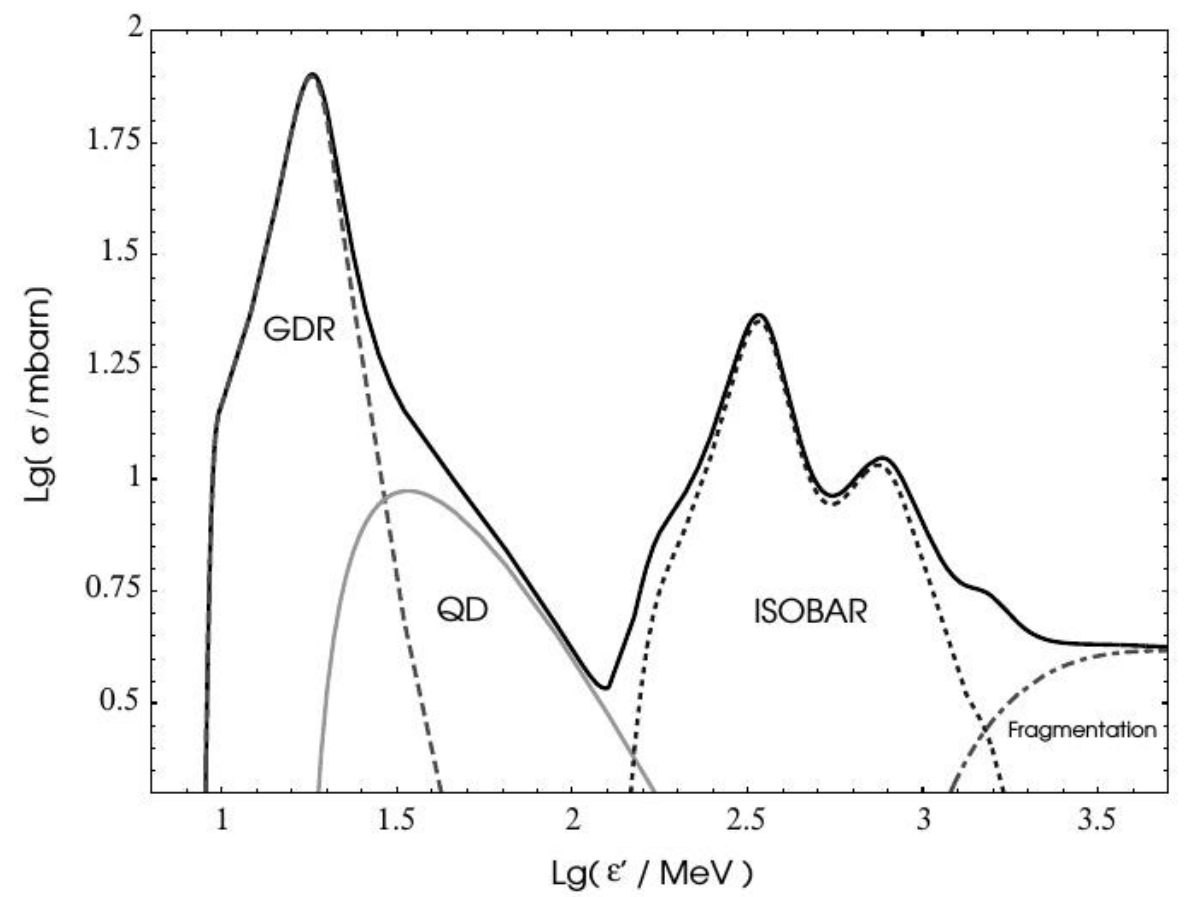

Figura 3.7 - Seção de choque da interação núcleo de ferro-fótons em função da energia do fótons no sistema de referência do próton $E_{\gamma}^{S R P C}=\epsilon^{\prime}$. Fonte: Figura extraída da referência (40). 


\subsection{UHECR produzindo fótons de GeV-TeV}

UHECRs interagem com a radiação de fundo no Universo produzindo partículas segundarias: píons, elétrons, pósitrons, raios gamas, neutrinos e nêutrons. Píons e nêutrons decaem antes de chegarem na Terra. Raios gamas interagem com a radiação de fundo e produzem pares elétron-pósitron. Elétrons e pósitrons podem espalhar fótons da radiação de fundo através do mecanismo Compton inverso aumentando a energia dos fótons espalhados. Raios gamas, elétrons, pósitrons e neutrinos são as partículas mais abundantes nesta cascata.

A cascata gera um alto fluxo de fótons secundários com energia entre GeV-TeV. Assim, o número de raios gamas medido em qualquer direção no céu é uma combinação dos fótons produzidos nas fontes (fluxo primário) com os fótons produzidos durante a propagação de UHECRs (fluxo secundário). Tem sido proposto que o brilho acentuado em raios gama com energia entre $\mathrm{GeV}-\mathrm{TeV}$ de blazares distantes é consequência da soma de um fluxo primário com um fluxo secundário gerado na propagação de prótons emitidos pela fonte (94-96).

A ambiguidade entre fluxo primário e secundários pode ser resolvida se o espectro intrínseco da fonte fosse conhecido. Ou então, para algumas fontes medidas por observatórios de raios gama, apenas um limite superior de raios gama é obtido. Nestes casos, o limite superior de raios gamas restringe a soma dos fluxos primários e secundários, podendo ser interpretado como um limite superior da emissão secundária em uma abordagem conservadora que despreza o fluxo primário.

O limite superior do fluxo secundário de raios gamas pode ser utilizado para calcular um limite superior no fluxo de UHECR que o produziu. Este limite no fluxo de UHECR refere-se a emissão de uma fonte pontual já que os fótons produzidos não são desviados por campos magnéticos. Esta técnica permite estudar fontes pontuais de UHECR através da detecção de fótons com energia entre $\mathrm{GeV}-\mathrm{TeV}$, fato impossível de ser feito até o momento com a medida direta dos UHECRs já que estes são desviados nos campos magnéticos. Na seção seguinte, detalhamos os cálculos e modelagem desta proposta. 


\subsubsection{Limite na luminosidade de fonte pontuais de UHECR}

Considerando uma fonte que emita UHECR com espectro de energia dado por uma lei de potência com um corte exponencial

$$
\frac{d N}{d E d t}=\frac{L_{C R}}{C_{0}} E^{-\alpha} \exp \left(-E / E_{c u t}\right),
$$

onde $L_{C R}$ é a luminosidade da fonte em UHECR, $\alpha$ é o índice espectral, $E_{c u t}$ é a energia de corte e $C_{0}$ é uma constante de normalização

$$
C_{0}=\int_{E_{\min }}^{\infty} d E E^{-\alpha+1} \exp \left(-E / E_{\text {cut }}\right),
$$

na qual $E_{\min }=10^{18} \mathrm{eV}$ é a energia mínima de um raio cósmico produzido na fonte.

O espectro na fonte pode ser reescrito como

$$
\frac{d N}{d E d t}=\frac{L_{C R}}{\langle E\rangle_{0}} P_{C R}^{0}(E)
$$

onde $P_{C R}^{0}(E)$ é a distribuição normalizada de partículas injetadas pela fonte.

$$
P_{c r}^{0}(E)=\frac{E^{-\alpha} \exp \left(-E / E_{c u t}\right)}{\int_{E_{\text {min }}}^{\infty} d E E^{-\alpha} \exp \left(-E / E_{c u t}\right)}
$$

e $\langle E\rangle_{0}$ é a energia média

$$
\langle E\rangle_{0}=\int_{E_{\min }}^{\infty} d E E P_{C R}^{0}(E)=\frac{\int_{E_{\min }}^{\infty} d E E^{-\alpha+1} \exp \left(-E / E_{c u t}\right)}{\int_{E_{\min }}^{\infty} d E E^{-\alpha} \exp \left(-E / E_{c u t}\right)}
$$

No caminho da fonte até a Terra, os raios cósmicos interagem com a radiação de fundo o que causa modificações na distribuição de energia de $P_{C R}^{0}(E)$ para $P_{C R}(E)$. Para uma fonte a uma dada distância comóvel $\left(D_{s}\right)$ da Terra, assumindo uma emissão isotrópica, o fluxo pode ser escrito como

$$
I_{C R}(E)=\frac{L_{C R}}{4 \pi D_{s}^{2}\left(1+z_{s}\right)\langle E\rangle_{0}} K_{C R} P_{C R}(E),
$$

onde $z_{s}$ é o redshift da fonte, $P_{C R}(E)$ é a distribuição de energias chegando na fonte e $K_{C R}$ é o número de raios cósmicos que chega na Terra por partícula injetada pela fonte. $K_{C R}$ e $P_{C R}(E)$ representam a física de propagação dos raios cósmicos incluindo todos as interações relevantes. 
Esta fonte gera uma fluxo secundário de raios gamas que é proporcional à luminosidade de raios cósmicos. Em analogia à equação 3.3.6 é possível escrever o fluxo de raios gama na Terra como uma função da luminosidade de raios cósmicos

$$
I_{\gamma}\left(E_{\gamma}\right)=\frac{L_{C R}}{4 \pi D_{s}^{2}\left(1+z_{s}\right)\langle E\rangle_{0}} K_{\gamma} P_{\gamma}\left(E_{\gamma}\right),
$$

na qual $K_{\gamma}$ é o número de raios gamas gerados por raio cósmico injetado pela fonte e $P_{\gamma}\left(E_{\gamma}\right)$ é a distribuição de energia de raios gamas que atinge a Terra. $E_{\gamma}$ é usado para denotar a energia os raios gamas. Note que $K_{\gamma}$ e $P_{\gamma}\left(E_{\gamma}\right)$ representam a física de propagação de raios cósmicos e raios gamas.

A equação 3.3.7 relaciona o fluxo de raios gama medidos na Terra com a luminosidade de raios cósmicos de forma que um limite superior no fluxo integral de raios gamas pode ser traduzido para um limite superior na luminosidade de raios cósmicos $\left(L_{C R}^{U L}\right)$ pela equação

$$
L_{C R}^{U L}=I_{\gamma}^{U L}\left(>E_{\gamma}^{t h}\right) \frac{4 \pi D_{s}^{2}\left(1+z_{s}\right)\langle E\rangle_{0}}{K_{\gamma} \int_{E_{\gamma}^{t h}}^{\infty} d E P_{\gamma}\left(E_{\gamma}\right)},
$$

onde $I_{\gamma}^{U L}\left(>E_{\gamma}^{t h}\right)$ é o limite superior no fluxo integral de raios gamas obtido acima de uma certa energia de limiar $\left(E_{\gamma}^{t h}\right)$ em um certo nível de confiança ( $C L$ - Confidence Level).

A equação 3.3.8 resume o método por nós elaborados na referência (41). $K_{\gamma}$ e $P_{\gamma}\left(E_{\gamma}\right)$ incluem todos os efeitos de propagação de uma fonte injetando um espectro $P_{C R}^{0}(E)$ a uma distância $D_{S}$ da Terra. Os fatores $K_{C R}$ e $K_{\gamma}$ e as distribuições $P_{C R}(E)$ e $P_{\gamma}\left(E_{\gamma}\right)$ podem ser obtidos através de simulações de $M C$ da trajetória das partículas na radiação de fundo.

Para uma ilustração da aplicação deste método consideremos o estudo de Pictor A. Pictor A é uma radio galáxia do tipo Fanarof-Riley II (97). Dado sua relativa proximidade $(z=0.035)$ ela tem sido alvo de extensivas campanhas de medidas em vários comprimentos de onda. Recentemente, o satélite FERMI-LAT detectou raios gamas com $(0.2<E<300 \mathrm{GeV})$ com fluxo dados por $\left(F_{0.2<E<300 \mathrm{GeV}}=(5.8 \pm 0.7) \times 10^{-9} \mathrm{~cm}^{-2} \mathrm{~s}^{-1}\right)(98)$.

A abundância de dados desta fonte (veja referência (99)) e a possibilidade de resolver sua estrutura interna tornou Pictor A um candidato preferencial na abordagem de várias questões relativas a emissão de raios gamas. A emissão em amplo espectro é sugestiva de que mecanismos self-Compton estão operando na fonte o que indicaria a produção de raios gama com $\mathrm{TeV}$ através de espalhamento Compton inverso. No entanto, o fluxo previsto por este modelo é bem mais baixo do que as medidas realizadas por FERMI-LAT e H.E.S.S. (98).

Relativo a produção de UHECRs, Pictor A é também um candidato plausível. Um campo 
magnético da ordem de $1 \mu$ Gauss foi medidos nos filamentos com tamanho de kpc (100) o que garante a possibilidade de aceleração de partículas com energia $E \sim 10^{20} \mathrm{eV}$ em acordo com o critério de Hillas (101). Ao mesmo tempo, a luminosidade do jato $L_{\text {jet }} \sim 10^{43.1} \mathrm{erg}$ $\mathrm{s}^{-1}$ também satisfaz a condição de Lovelace (102).

O limite superior no fluxo integral de raios gamas de Pictor A obtido por H.E.S.S., a $99.9 \% \mathrm{CL}$, é $I_{\gamma}^{U L}\left(E>E_{\gamma}^{t h}=320 \mathrm{GeV}\right)=2.45 \times 10^{-12} \mathrm{~cm}^{-2} \mathrm{~s}^{-1}$. Usaremos este limite medido por H.E.S.S. e a equação 3.3.8 para calcular o limite superior na luminosidade de raios cósmicos de altas energias emitidos por Pictor $A$.

Simulamos a propagação de partículas cósmicas de Pictor A até a Terra com o software CRPropa (103). CRPropa é um dos mais completos softwares públicos de propagação de partículas no meio intergalático que considera a interação das partículas com a radiação de fundo em detalhes. CRPropa também propaga as partículas e fótons secundários geradas pelo fluxo primário. Consideramos $\alpha=2.3$ e dois casos onde só prótons ou só núcleos de ferro são emitidos pela fonte. As energias de corte consideradas foram $E_{c u t}^{p}=10^{20.5} \mathrm{eV} \mathrm{e}$ $E_{\text {cut }}^{26}=26 \times 10^{20.5} \mathrm{eV}$. Para obtermos uma boa descrição das flutuações envolvidas simulamos $10^{7}$ saindo de Pictor A. A propagação das partículas foi feita unidimensionalmente desprezando os efeitos da interação com o campo magnético. Os efeitos do campo magnético estão sendo avaliados em um trabalho em preparação.

A figura 3.8 mostra o resultado das simulações. O fluxo de raios cósmicos e raios gamas na Terra foi calculado a partir de um fluxo de raios cósmicos na fonte permitindo então o cálculo dos fatores e distribuições $K$ e $P$ mencionados acima.

A figura 3.9 mostra a contribuição do limite superior de UHECR emitidos por Pictor A ao fluxo total medido pelo Observatório Pierre Auger (104) para vários casos. Os gráficos na coluna esquerda foram feitos com a suposição de emissão de prótons. Os gráficos na coluna direita foram feitos com a suposição de emissão de núcleos de ferro. Cada linha mostra um corte superior de energia $\log \left(E_{\text {cut }}^{p r} / \mathrm{eV}\right)=20,20.5$ e 21 para prótons e $\log \left(E_{\text {cut }}^{26} / \mathrm{eV}\right) \cong$ $21.41,21.91$ e 22.41 para núcleos de ferro $\left(E_{c u t}^{26}=26 \times E_{p r}\right)$. Note que o fluxo calculado deve ser pesado pela exposição do Observatório Pierre Auger na direção de Pictor A tal como explicado no anexo B da referência (41).

Na figura 3.9 (coluna da direita) é possível ver que uma série de combinações de energias de corte e índice espectral ultrapassam o fluxo medido pelo Observatório Pierre Auger em vários intervalos de energia. Para esses casos, o limite no fluxo de raios gamas medido pelo Observatório H.E.S.S. não é restritivo o suficiente para impor um limite no fluxo de UHECR. 


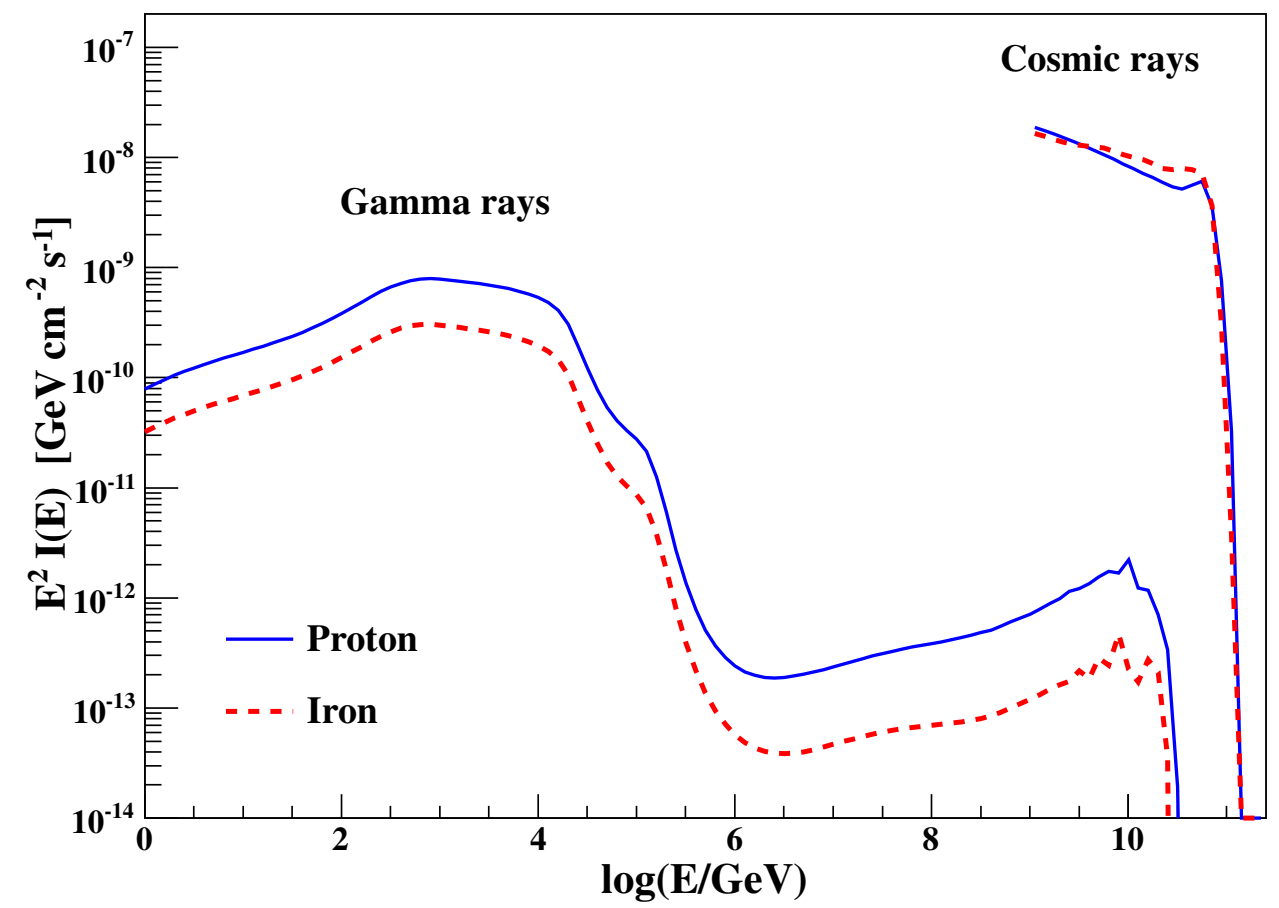

Figura 3.8 - Fluxo de raios cósmicos e raios gamas na Terra em função do logaritmo da energia para Pictor A emitindo um espectro de lei de potência com características dada no texto. Linhas cheias correspondem à injeção de próton e linhas tracejadas à injeção de núcleo de ferro. Fonte: Figura extraída da referência (41).

Por outro lado, a coluna da esquerda da figura 3.9 mostra que a medida realizada por H.E.S.S. oferece um limite válido de UHECR se prótons são considerados saindo da fonte. Para todos os casos considerados, o limite de UHECR calculado é inferior ao fluxo medido pelo Observatório Pierre Auger. A figura 3.10 mostra o limite superior da luminosidade de prótons de Pictor A em função do índice espectral para $\log \left(E_{\text {cut }} / \mathrm{eV}\right)=20,20.25,20.5,20.75$, e 21 .

Núcleos propagando pelo Universo emitem mais gamas que prótons. Desde forma, o calculo acima para Pictor A representa apenas um limite superior da luminosidade de prótons. Por outro lado, se um limite superior for imposto usando os fatores e distribuições de propagação $\left(P_{\gamma}^{F e}\right.$ e $\left.K_{\gamma}^{F e}\right)$ para núcleos de ferro, este limite vincula o fluxo total de todos os UHECR emitidos por aquela fonte. Para detalhes veja o apêndice A da referência (42).

Invertendo o raciocínio acima, é possível partir do fluxo medido pelos Observatório de UHECR e calcular qual o limite na integral do fluxo de raios gama $\left(I_{\gamma}^{\max }\left(E_{\gamma}, \hat{n}\right)\right)$ necessário para impor um limite no fluxo de UHECR válido

$$
I_{\gamma}^{\max }\left(E_{\gamma}, \hat{n}\right)=\frac{C_{s}^{\max }}{W_{s}(\hat{n}) 4 \pi D_{s}^{2}\left(1+z_{s}\right)\langle E\rangle_{0}} K_{\gamma} P_{\gamma}\left(E_{\gamma}\right)
$$

na qual $C_{s}^{\max }=L_{C R} W_{s}$ é o fluxo de UHECR medido.

A figura 3.11 mostra o limite da integral do fluxo de raios gamas $\left(I_{\gamma}^{\max }\left(E_{\gamma}, \hat{n}\right)\right)$ necessário 


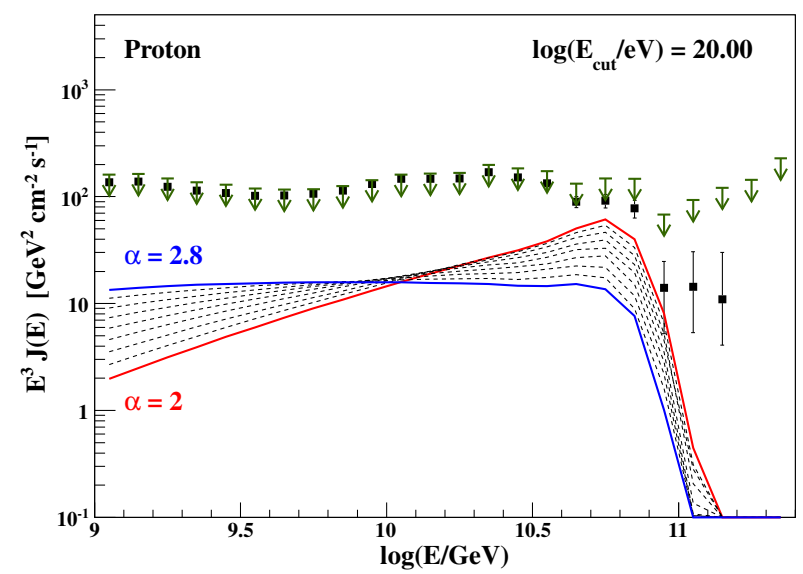

(a) $\operatorname{Pr}-E_{\text {cut }}=10^{20} \mathrm{eV}$

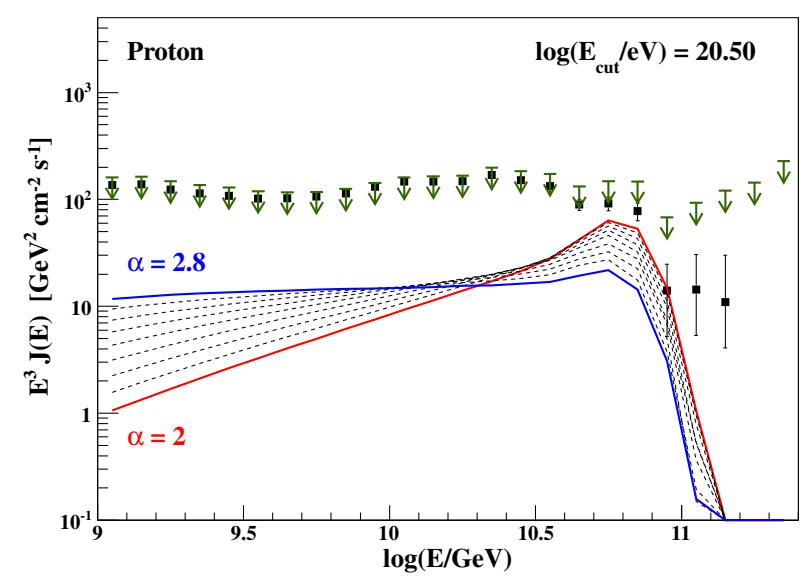

(c) $\operatorname{Pr}-E_{\text {cut }}=10^{20.5} \mathrm{eV}$

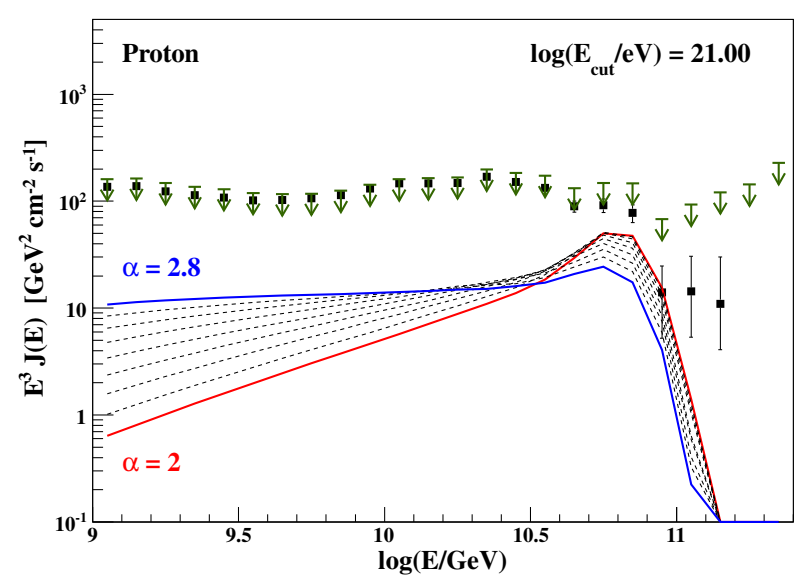

(e) $\operatorname{Pr}-E_{\text {cut }}=10^{21} \mathrm{eV}$

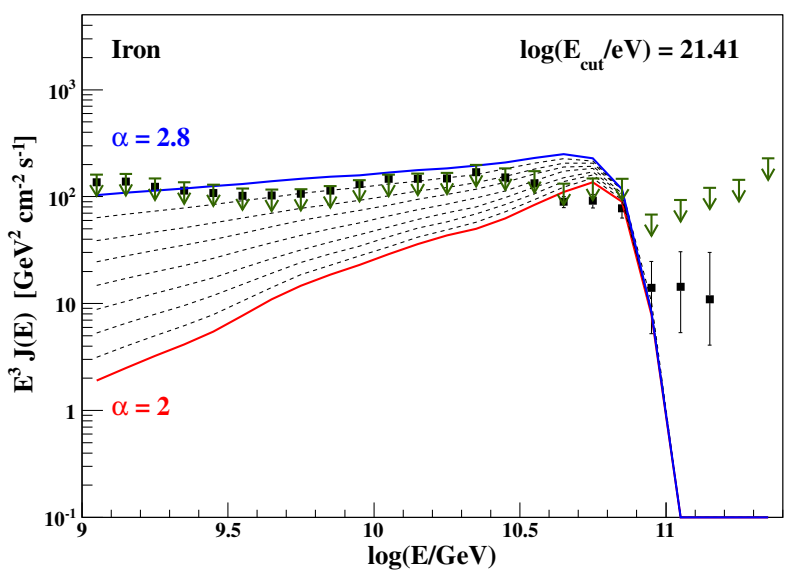

(b) $\mathrm{Fe}-E_{\text {cut }}=26 \times 10^{20} \mathrm{eV}$

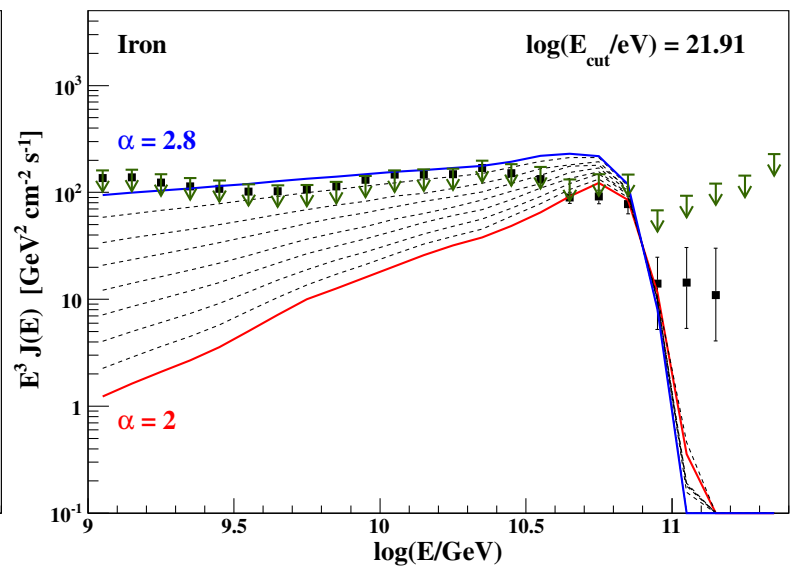

(d) $\mathrm{Fe}-E_{\text {cut }}=26 \times 10^{20.5} \mathrm{eV}$

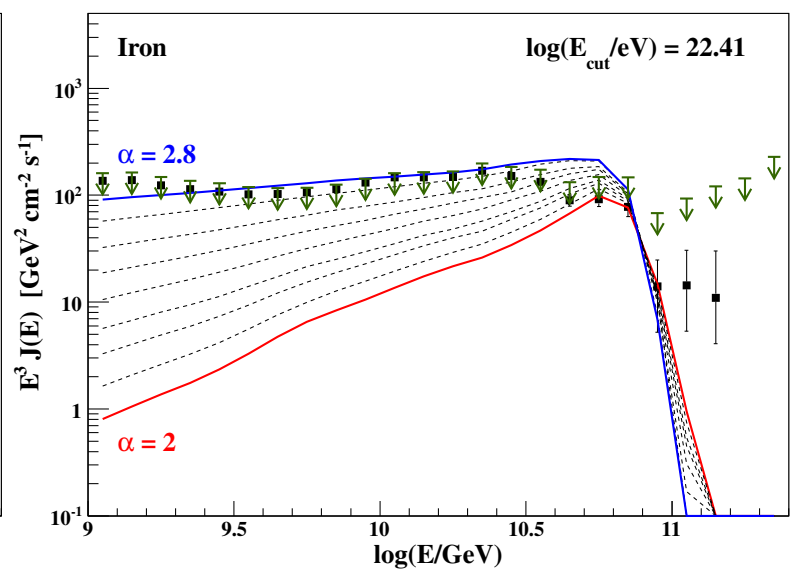

(f) $\mathrm{Fe}-E_{\text {cut }}=26 \times 10^{21} \mathrm{eV}$

Figura 3.9 - Limite superior de UHECR produzidos por Pictor A de acordo com cálculo baseado no limite superior da integral do fluxo de raios gama com energia entre GeV-TeV medidos pelos telescópios H.E.S.S.. Os pontos corresponder ao espectro de energia medido pelo Observatório Pierre Auger . Coluna da esquerda: Figuras (a), (c), e (e) correspondem a prótons primários. Coluna da direita: Figuras (b), (d), e (f) correspondem a núcleos de ferro primários. Fonte: Figura extraída da referência (41). 


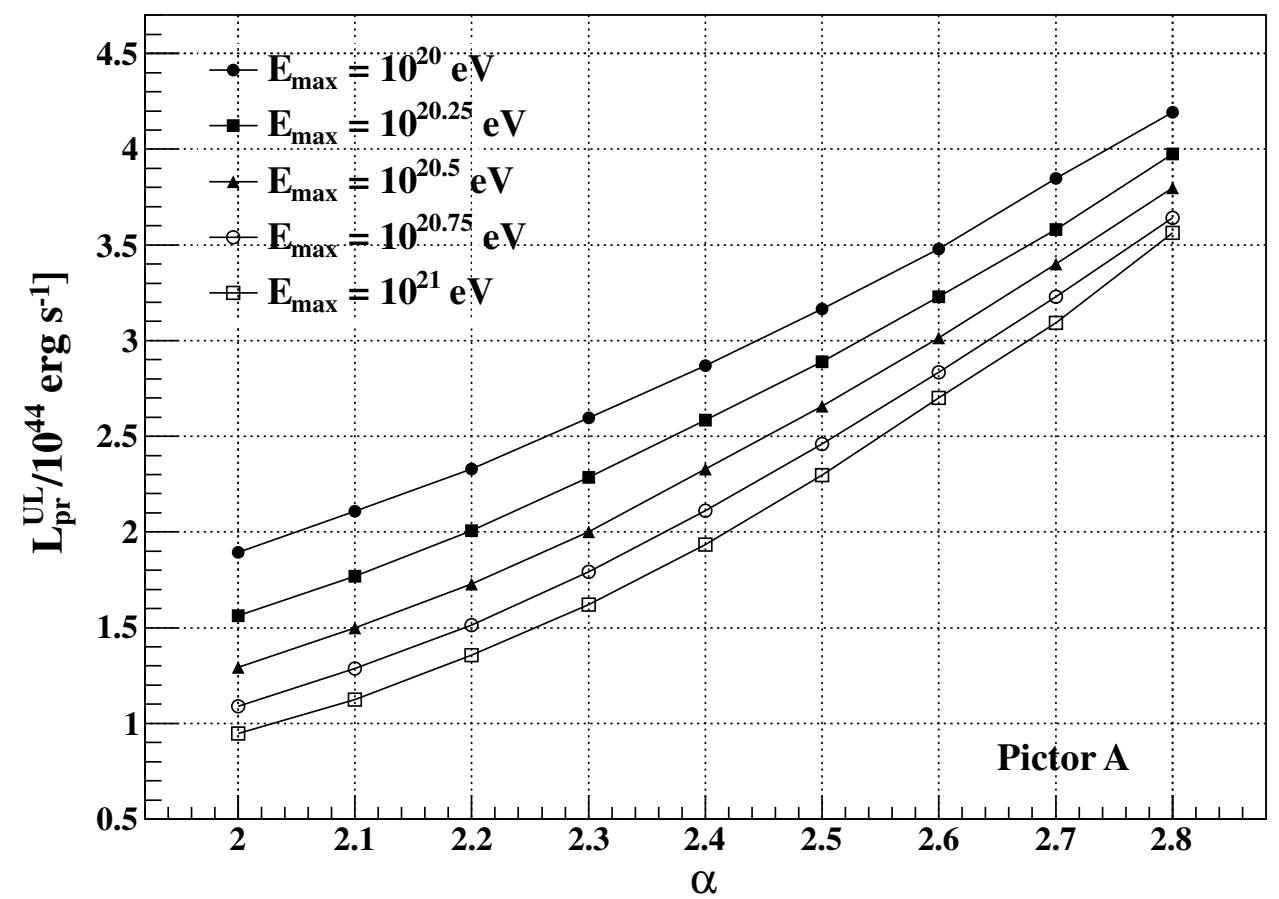

Figura 3.10 - Limite superior na luminosidade de prótons emitidos por Pictor A em função do índice espectral de injeção. Os cálculos foram obtidos com base no limite do fluxo integral de raios gama com energia $\mathrm{GeV}-\mathrm{TeV}$ medidos pelo H.E.S.S.. As energias de corte consideradas são $\log \left(E_{\text {cut }} / \mathrm{eV}\right)=20,20.25,20.5$, 20.75, e 21. Fonte: Figura extraída da referência (41).

para impor um limite válido na luminosidade de UHECR em função da distância da fonte para três casos de energia dos fótons. As linhas vermelhas (azul) correspondem ao valor de $I_{\gamma}^{\max }\left(E_{\gamma}, \hat{n}\right)$ para o caso de prótons (núcleos de ferro) emitidos pela fonte. Os espectros de UHECR medidos pelos Observatório Auger e TA foram utilizados. Dependo da posição da fonte no céu, Auger ou TA oferecem um limite mais restritivo. As setas mostram fontes medidas pelo FERMI-LAT. Se a seta está posicionada abaixo das linhas azuis, significa que a medida do FERMI-LAT é suficiente para limitar na luminosidade de prótons daquela fonte. Se a seta está posicionada abaixo das linhas vermelhas, os dados do FERMI-LAT impõem um limite na luminosidade de todos os UHECR emitidos por aquela fonte.

As tabelas 3.1,3.2 e 3.3 resumem todas as fontes estudadas com esta técnica até o momento com os respectivos limites na luminosidade de UHECR. 


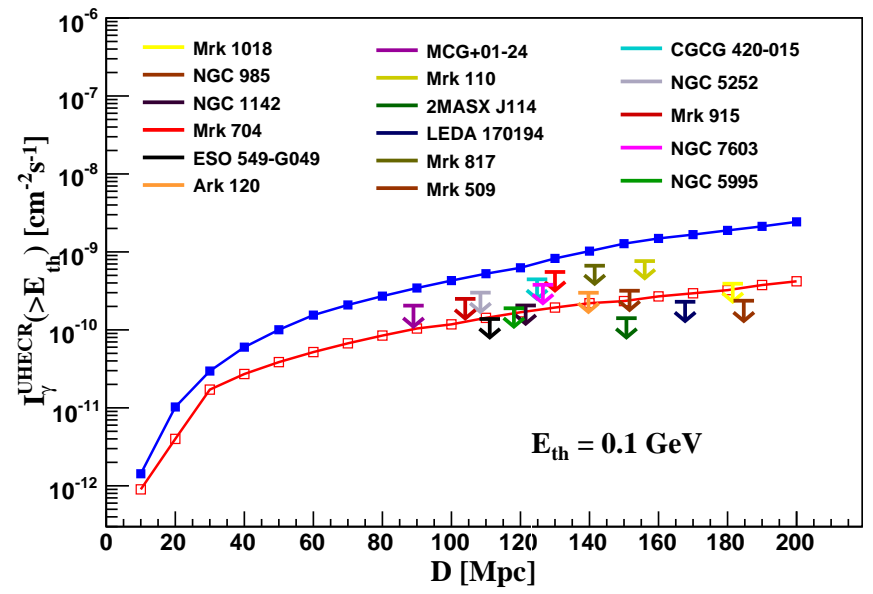

(a) Fermi-LAT- TA - $E_{t h}=0.1 G e V$

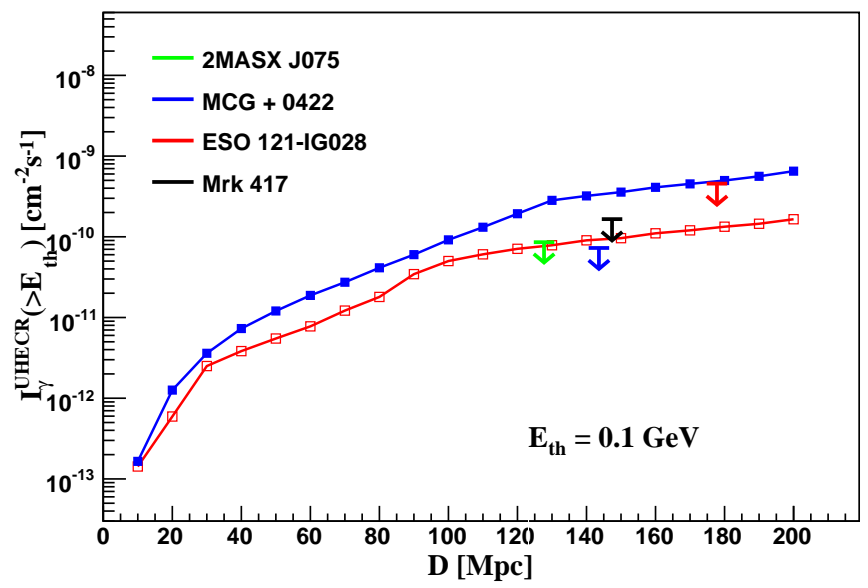

(b) Fermi-LAT- Auger - $E_{t h}=0.1 \mathrm{GeV}$

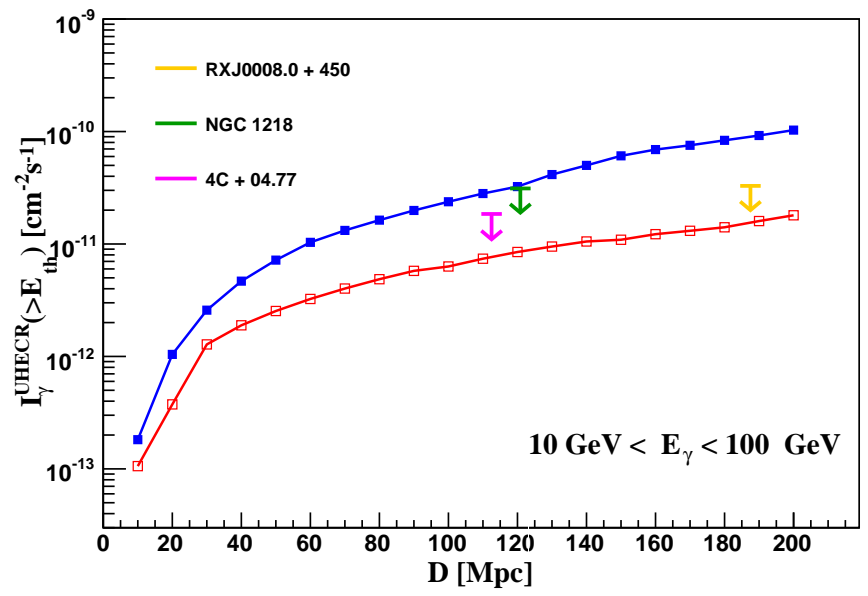

(c) Fermi-LAT- TA - $10<E_{\gamma}<100 \mathrm{GeV}$

Figura 3.11 - Limite superior da integral do fluxo de raios gama $\left(I_{\gamma}^{U H E C R}\right.$ a $\left.95 \% \mathrm{CL}\right)$ em função da distância da fonte. As linhas vermelhas com quadrados abertos correspondem a núcleos de ferro primário. As linhas azuis com quadrados fechados correspondem a prótons primários. As setas correspondem aos dados medidos pelos observatórios de raios gama multiplicados pela exposição $\left(W_{s}\right)$ correspondente. Para todos os casos consideramos $\alpha=2.4$ e $E_{\text {cut }}=Z \times 10^{10.5} \mathrm{eV}$. Em cada gráfico a legenda mostra o Observatório que mediu os raios gamas (FERMI-LAT), o Observatório de UHECR (Auger ou TA) e a energia dos fótons usada para calcular o limtes no fluxo. Fonte: Figura extraída da referência (42). 
Tabela 3.1 - A coluna 1 mostra o nome das fontes. A coluna 2 e 3 são os dados retirados das referências $(57,58)$ que mostram a distância da fonte à Terra e o limite superior do fluxo de raios gama $(E>0.1 \mathrm{GeV})$ com $95 \% \mathrm{CL}$ medido pelo FermI-LAT. A coluna 4 mostra o limite superior na luminosidade de prótons calculado para cada fonte. A coluna 5 mostra o limite superior da luminosidade total de UHECR para os casos em que foi possível fazer o cálculo.

\begin{tabular}{|c|c|c|c|c|}
\hline Nome da fonte & $\mathbf{D}[\mathrm{Mpc}]$ & $\begin{array}{l}\text { UL: } \mathcal{F}(>0.1 \mathrm{GeV}) \\
{\left[10^{-9} \mathrm{ph} \mathrm{cm}^{-2} \mathrm{~s}^{-1}\right]}\end{array}$ & $\begin{array}{l}\mathbf{L}_{\mathbf{p r}}^{\mathbf{U L}} \text { (Próton) } \\
{\left[\mathrm{erg} \mathrm{s}^{-1} \times 10^{45}\right]}\end{array}$ & $\begin{array}{c}\mathbf{L}_{\mathbf{C R}}^{\mathbf{U L}}(\text { Total) } \\
{\left[\mathrm{erg} \mathrm{s}^{-1} \times 10^{45}\right]}\end{array}$ \\
\hline Mrk 1018 & 181.5 & 2.1 & 1.04 & - \\
\hline NGC 985 & 184.7 & 1.8 & 1.03 & 2.19 \\
\hline NGC 1142 & 121.5 & 1.1 & 0.49 & - \\
\hline 2MASX J07595347+2323241 & 127.7 & 2.2 & 1.01 & - \\
\hline Mrk 704 & 130 & 2.0 & 0.91 & - \\
\hline MCG+04-22-042 & 143.6 & 1.6 & 0.75 & 1.74 \\
\hline Mrk 417 & 147.4 & 3.7 & 1.72 & - \\
\hline ESO 121-IG028 & 177.8 & 1.2 & 0.64 & - \\
\hline ESO 549-G049 & 111.1 & 2.5 & 1.11 & - \\
\hline CGCG 420-015 & 124.8 & 2.1 & 0.95 & - \\
\hline Ark 120 & 139.7 & 1.6 & 0.74 & - \\
\hline MCG-01-24-012 & 89.0 & 1.5 & 0.65 & - \\
\hline Mrk 110 & 156 & 1.9 & 0.90 & - \\
\hline 2MASX J11454045-1827149 & 150.7 & 2.8 & 1.30 & 3.16 \\
\hline LEDA 170194 & 167.7 & 3.1 & 1.48 & 3.71 \\
\hline NGC 5252 & 108.4 & 1.4 & 0.62 & - \\
\hline Mrk 817 & 141.5 & 1.6 & 1.72 & - \\
\hline NGC 5995 & 118.1 & 2.0 & 0.90 & - \\
\hline Mrk 509 & 151.6 & 2.7 & 1.27 & - \\
\hline Mrk 520 & 115.5 & 2.2 & 0.98 & - \\
\hline Mrk 915 & 104 & 2.4 & 1.06 & - \\
\hline NGC 7603 & 126.5 & 2.0 & 0.91 & - \\
\hline
\end{tabular}


Tabela 3.2 - A coluna 1 mostra o nome das fontes. A coluna 2 e 3 são os dados retirados das referências (59-61) que mostram a distância da fonte à Terra e o limite superior do fluxo de raios gama com 99\% CL VERITAS, 95\% CL MAGIC e 99\%CL H.E.S.S. acima de $E_{t h}$. A coluna 4 mostra a energia de limiar das medidas $\left(E_{t h}\right)$. A coluna 5 mostra qual observatório detectou a fonte. A coluna 6 mostra o limite superior na luminosidade de prótons calculado para cada fonte. A coluna 7 mostra o limite superior da luminosidade total de UHECR para os casos em que foi possível fazer o cálculo.

\begin{tabular}{|c|c|c|c|c|c|c|}
\hline Nome da Fonte & $\mathbf{D}[\mathrm{Mpc}]$ & $\begin{array}{c}\mathbf{U L :} \mathcal{F}\left(>E_{t h}\right) \\
{\left[\mathrm{ph} \mathrm{cm}^{-2} s^{-1}\right]}\end{array}$ & $\begin{array}{c}\mathbf{E}_{\mathbf{t h}} \\
{[\mathrm{GeV}]}\end{array}$ & Medido por & $\begin{array}{c}\mathbf{L}_{\mathbf{p r}}^{\mathbf{U L}} \text { (Próton) } \\
{\left[\mathrm{erg} \mathrm{s}^{-1} \times 10^{45}\right]}\end{array}$ & $\begin{array}{c}\mathbf{L}_{\mathbf{C R}}^{\mathbf{U L}}(\text { Total) } \\
{\left[\mathrm{erg} \mathrm{s}^{-1} \times 10^{45}\right]}\end{array}$ \\
\hline Pictor A & 149 & $2.45 \times 10^{-12}$ & 330 & H.E.S.S. & 1.6 & - \\
\hline NGC 7469 & 60 & $1.38 \times 10^{-12}$ & 330 & H.E.S.S. & 0.2 & - \\
\hline 3C 111 & 196 & $2.5 \times 10^{-12}$ & 300 & VERITAS & 0.32 & 0.87 \\
\hline NGC 1275 & 75 & $5.11 \times 10^{-12}$ & 190 & VERITAS & 0.13 & - \\
\hline IC 310 & 78.5375 & $3.1 \times 10^{-12}$ & 300 & MAGIC & 0.11 & - \\
\hline
\end{tabular}

Tabela 3.3 - A coluna 1 mostra o nome das fontes. A coluna 2 e 3 são os dados retirados das referências $(57,58)$ que mostram a distância da fonte à Terra e o limite superior do fluxo de raios gama $\left(10<E_{\gamma}<100 \mathrm{GeV}\right)$ com $95 \% \mathrm{CL}$ tais como medido pelo FeRMI-LAT. A coluna 4 mostra o limite superior na luminosidade de prótons calculado para cada fonte. Para essas fontes não foi possível calcular um limite no fluxo de UHECR.

\begin{tabular}{|c|c|c|c|}
\hline Nome da fonte & $\mathbf{D}[\mathrm{Mpc}]$ & $\begin{array}{c}\text { UL: } \mathcal{F}(10<E<100 \mathrm{GeV}) \\
{\left[\mathrm{ph} \mathrm{cm}^{-2} \mathrm{~s}^{-1}\right]}\end{array}$ & $\begin{array}{c}\mathbf{L}_{\mathrm{CR}}^{\mathrm{UL}} \text { (Próton) } \\
{\left[\mathrm{erg} \mathrm{s}^{-1} \times 10^{45}\right]}\end{array}$ \\
\hline NGC 1218 & 120.83 & $1.47 \times 10^{-10}$ & 1.27 \\
\hline $4 \mathrm{C}+04.77$ & 112.5 & $8.59 \times 10^{-11}$ & 0.31 \\
\hline RX J0008.0+1450 & 187.5 & $1.22 \times 10^{-10}$ & 1.36 \\
\hline
\end{tabular}




\section{Geração de Raios Cósmicos de Altíssimas Energias}

A geração de partículas com energia superior a $10^{19} \mathrm{eV}$ representa um desafio para a física e astrofísica atuais. Não há um objeto no Universo operando um mecanismos conhecido que tenha sido identificado como fonte inquestionável de UHECR com energia superior a $10^{19} \mathrm{eV}$. Os modelos de geração propostos não são totalmente satisfatórios pois não podem ser deduzidos de primeiros princípios, dependendo assim de vários parâmetros ajustáveis aos dados.

O modelo fundamental de aceleração de partículas em ambientes astrofísicos foi proposto por Enrico Fermi em 1949 (105). A ideia básica exposta no artigo é que partículas carregadas colidem com nuvens magnetizadas de plasma em movimento ganhando em média uma quantidade de energia proporcional ao quadrado da velocidade da nuvem a cada interação (Mecanismo de Fermi de Segunda Ordem). Sucessivos encontros com nuvens em movimento aumentam a energia da partícula até os valores medidos na Terra. O desenvolvimento desta ideia original utilizando ondas de choque magnetizadas resulta em um ganho de energia proporcional à velocidade da onda e portanto ficou conhecido como Mecanismo de Fermi de Primeira Ordem (106-110). Um dos sucessos deste modelo é a reprodução de um espectro de energia em lei de potência. Pequenas variações destes mecanismos são utilizadas para tentar descrever a aceleração de partículas em ambientes astrofísicos galácticos e extragalácticos.

Uma classe de modelos de aceleração chamada de top-down ficou muito popular entre os anos de 1990 e 2010. Inspirados em medidas do Observatório AGASA que mostravam uma continuação do espectro para energias além de $10^{19.5} \mathrm{eV}(111)$, estes modelos representavam uma alternativa à aceleração de partículas via Mecanismos de Fermi. A ideia fundamental destas propostas é que relíquias do Universo primordial produzidas logo após a inflação podem decair hoje e produzir UHECR. Para uma revisão desses modelos veja $(79,112)$. No entanto, os dados de anisotropia (6) e os limites no fluxo de fótons (71) e neutrinos (72) medidos pelo Observatório Pierre Auger tem reduzido muito, para valores praticamente desprezíveis, a importância de modelos top-down na composição do fluxo total de raios cósmicos. 


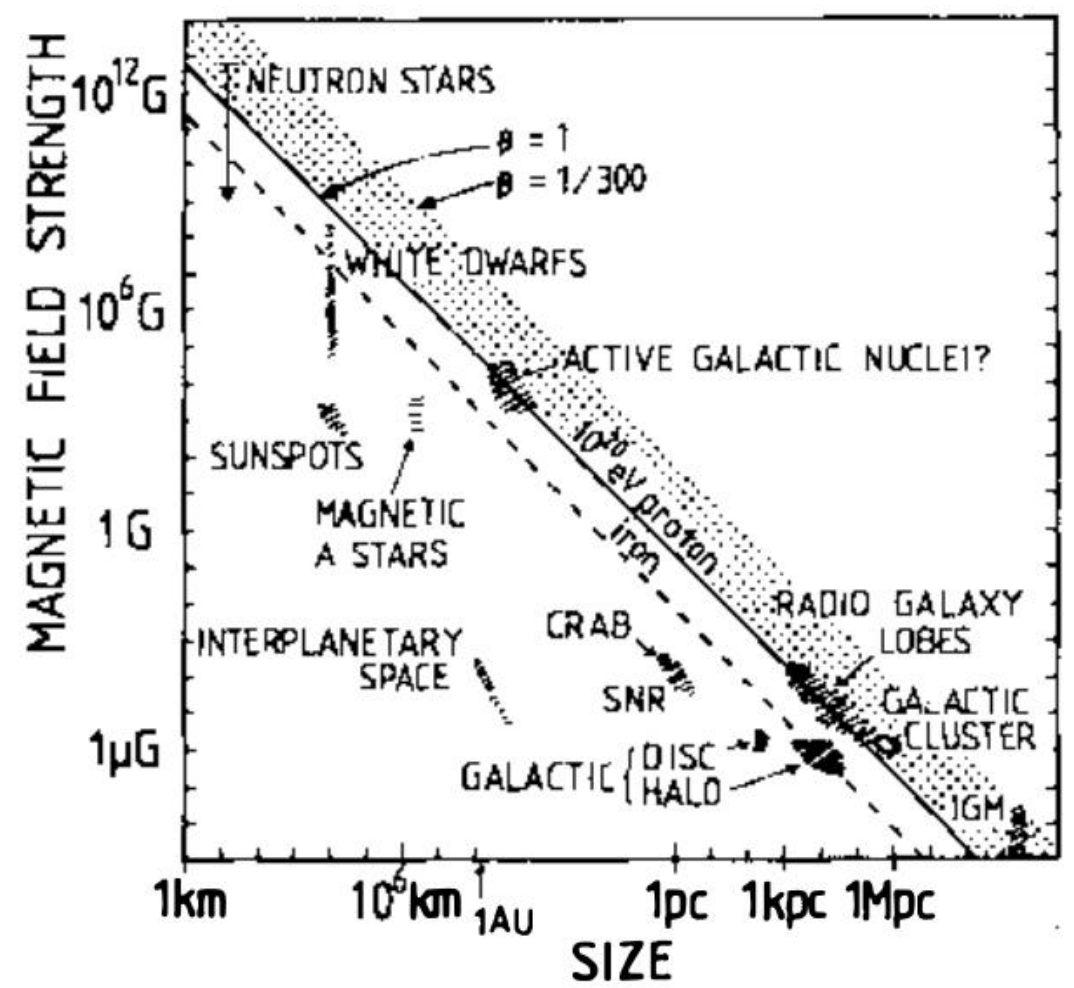

Figura 4.1 - Gráfico de Hillas. São mostrados a intensidade do campo magnético e o raio da fonte aceleradora para diversos tipos de objetos astrofísicos. As linhas diagonais são a solução da equação 4.0.2 para próton (linha cheia) e núcleo de ferro (linha tracejada) com $10^{20} \mathrm{eV}$. Fonte: Figura extraída da referência (43).

Modelos baseados em discos de acreção rodando ao redor de estrelas de nêutrons e buracos negros também tem sido propostos (102). No entanto, as altas densidades de radiação em torno do objeto compacto central impõem uma rápida perda de energia a partículas muito energéticas limitando a energia máxima à $\sim 10^{15} \mathrm{eV}(113)$. Apesar do insucesso, a ideia original de aceleração em dínamos com altas intensidades de campo elétrico pode ser utilizada como uma limitação do poder de aceleração em jatos. O limite imposto por esse modelo pode ser expresso por:

$$
L_{\text {jet }} \geq 10^{47} \mathrm{erg} \mathrm{s}^{-1} f_{\text {int }}\left(\frac{E_{\text {max }}}{Z \times 10^{21} \mathrm{eV}}\right)^{2},
$$

onde $f_{\text {int }}$ é um fator de intermitência descrevendo as flutuações temporais do fluxo energético, $E_{\max }$ é a energia máxima de uma partícula na fonte e $Z e$ a carga desta partícula.

A forma mais simples e interessante de representar a limitação de aceleração de fontes foi elaborada por M. Hillas (43). A figura 4.1 mostra o resumo do argumento, nela estão graficados a intensidade do campo magnético na fonte $(B)$ e o tamanho da fonte $(L)$ dos principais candidatos à região aceleradora de UHECR. Para que uma partícula sofra o efeito do 
mecanismos acelerador, ela deve permanecer um largo tempo na fonte. O campo magnético $B$ é o responsável por manter a partícula presa enquanto ela é acelerada. Nos mecanismos de Fermi são as colisões com nuvens ou ondas de choque magnetizadas que dão o ganho de energia. Neste contexto, a máxima energia que uma partícula pode ter sob a influência deste campo magnético sem escapar da fonte com tamanho $(L)$ é:

$$
E_{\max }^{E e V}=e Z \beta B_{\mu G} L_{k p c},
$$

na qual $B_{\mu G}$ é o campo em nano Gauss, $L_{k p c}$ o tamanho da fonte em kilo parsec, $Z e$ é a carga da partícula e $\beta=v / c$ da onda ou nuvem de choque e $E_{\max }^{\mathrm{EeV}}$ é a energia máxima da partícula em unidades de EeV. Em outras palavras, o raio de Larmor não pode ser maior do que a fonte.

O gráfico de Hillas seleciona estrela de nêutrons, núcleos ativos de galáxias (AGNs) e os lóbulos da galáxias rádio ativas como os principais candidatos à aceleradores de partículas a energias superiores a $10^{19} \mathrm{eV}$.

Usando como base os modelos de Fermi de primeira e segunda ordem e obedecendo às limitações impostas pelos argumentos de Lovelace e Hillas, vários trabalhos foram realizados de forma a especificar e detalhar os mecanismos de aceleração de partículas em objetos astrofísicos. Em geral, esses modelos nascem de argumentos astrofísicos sobre o funcionamento da fonte, extraem um fluxo de partículas que precisa ser propagado no Universo de acordo com o discutido no capítulo 3 para finalmente ser comparado com os dados experimentais mostrados no capítulo 2 .

Somente na última década, com o aprimoramento das técnicas observacionais, os modelos de aceleração de UHECR começaram a sofrer restrições importantes. As medidas do Observatório Pierre Auger excluem várias propostas teóricas feitas nas décadas anteriores, aponta novos rumos e impõem exigências para os modelos sendo elaborados no momento. Dentro deste contexto, retomamos um modelo originalmente proposto em 1993 por Stanev, Biermann e Gaisser (44) e estendemos sua validade. Nas seções seguintes deste capítulo, descrevemos o modelo original e as melhorias por nós propostas. 


\subsection{Modelo Stanev, Biermann e Gaisser - 1993}

O argumento original proposto por Stanev, Biermann e Gaisser em 1993 (44) supõem três sítios aceleradores de partículas: a) explosões de supernova no meio interestelar, b) explosões de supernova em um vento estelar e c) rádio galáxias. Segundo esta proposta apenas estas três componentes deveriam explicar os espectro de raios cósmicos até as mais altas energias.

Os pontos principais deste modelo estão resumidos a seguir. Em supernovas, as ondas de choque são altamente instáveis e sub-estruturadas de forma que o ganho de energia é determinado pela escala das instabilidades (114-116). As partículas leves (pr e um pouco de $\mathrm{He)} \mathrm{são} \mathrm{predominantemente} \mathrm{aceleradas} \mathrm{em} \mathrm{ondas} \mathrm{de} \mathrm{choque} \mathrm{deste} \mathrm{tipo} \mathrm{causadas} \mathrm{pela} \mathrm{explosão}$ de supernovas SN la de relativa baixa massa. Partículas mais pesadas $(A>2)$ são aceleradas em ondas de choque decorrentes da explosão de supernovas de alta massa no vento solar ejetado por essas estrelas antes da explosão (exemplo estrelas Wolf-Rayet). Estas explosões causam um espectro de energia em duas fases que no modelo original seria a causa da quebra no fluxo em energias da ordem de $10^{15} \mathrm{eV}$ conhecido como joelho. As duas fases são decorrentes da dependência angular do campo magnético da estrela. Rádio galáxias contribuem para aumentar a energia das partículas re-acelerando nos jatos as partículas produzidas na galáxia.

A figura 4.2 ilustra o espectro de energia produzido por essas componentes. A linha 1 é o espectro resultante da explosão da supernova no meio interestelar. A energia máxima de um raio cósmico pode alcançar em uma onda de choque de uma supernova levando em conta a expansão Sedov foi calculada na referência (117). O índice espectral tem valor aproximado de -2.75 e uma queda exponencial que pode ser escrita por:

$$
(d N / d E)_{1}=A_{1} \cdot E^{-2.75} \cdot \exp -E / E_{1}^{\text {cutoff }},
$$

onde $A_{1}$ é uma constante de normalização e $E_{1}^{\text {cutoff }}=10^{14.1} \mathrm{eV}$ na proposta original. $\mathrm{O}$ modelo também propõem que $E_{1}^{\text {cutoff }} \propto Z$.

O número 2 na figura 4.2 marca o espectro resultante da explosão de uma supernova no vento estelar (118). O espectro produzido tem índice espectral próximo de -2.67 para energias menores que $E_{2}^{\text {break }}$ e -3.07 para energias menores que $E_{2}^{\text {cutoff }}$ que é a determinação do corte exponencial do fluxo. $E_{2}^{\text {break }}$ e $E_{2}^{\text {cutoff }}$ são ambos proporcionais a carga da partícula. Esta componente pode ser escrita como: 


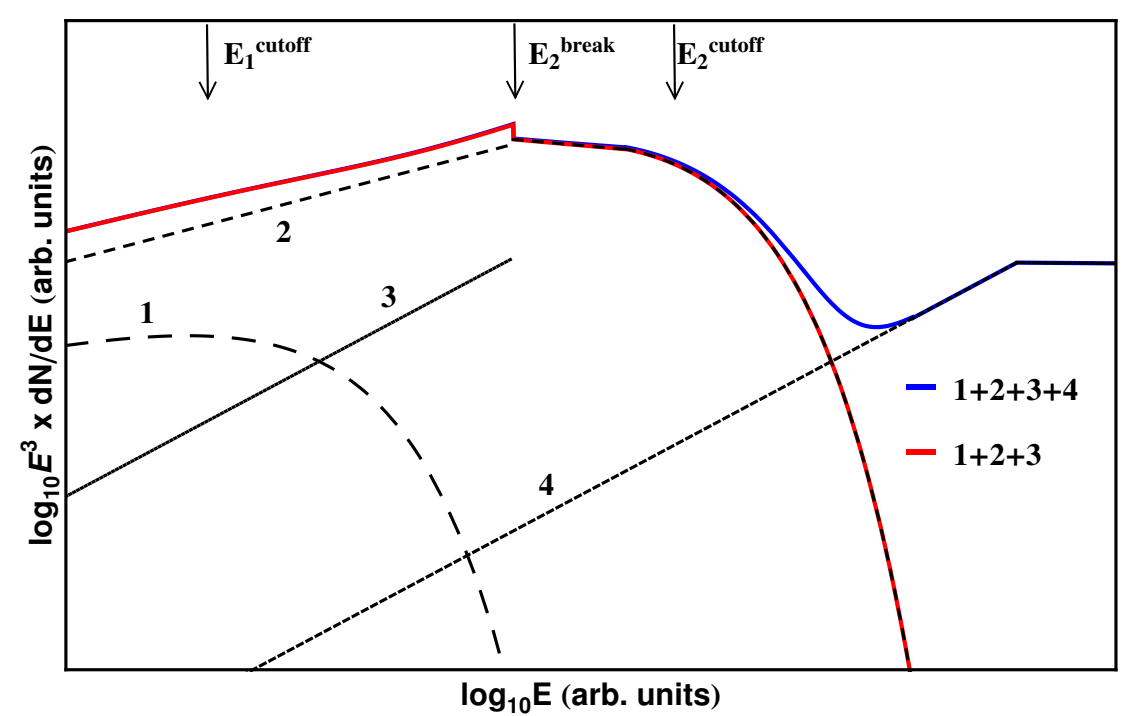

Figura 4.2 - Representação esquemática do espectro de energia proposto pela referência (44). Quatro curvas são mostradas: 1) explosão de supernovas no meio interestelar (fase Sedov), 2) explosão de supernova no vento estelar (Wolf-Rayet), 3) componente polar da explosão da supernova e 4) componente extragaláctica (rádio galáxias). Fonte: Figura extraída da referência (45).

$$
(d N / d E)_{2}=\left\{\begin{array}{ll}
A_{2} \cdot E^{-2.67} & \text { se } E<E_{2}^{\text {break }} \\
B_{2} \cdot E^{-3.07} \cdot \exp -E / E_{2}^{\text {cutoff }} & \text { se } E>E_{2}^{\text {break }}
\end{array},\right.
$$

onde $A_{2}$ e $B_{2}$ são constantes de normalização.

O número 3 na figura 4.2 marca uma componente extra resultante da explosão da supernova no vento estelar. No final da vida de estrelas massivas há uma conexão entre a rotação e o campo magnético que controla a explosão da estrela (119). Esta conexão produz uma componente polar de emissão de raios cósmicos que pode ser escrita como:

$$
(d N / d E)_{3}=A_{3} \cdot E^{-2.33} \text { se } E<E_{3}^{\text {cutoff }},
$$

na qual $A_{3}$ é uma constante de normalização, $E_{3}^{\text {cutoff } f}$ é a energia de corte e o índice espetral foi proposto da ordem de -2.33. Uma proposta simplificadora impõem que $E_{2}^{\text {break }}=E_{3}^{\text {cutoff }}$.

O número 4 na figura 4.2 mostra a contribuição extragaláctica produzida em rádio galáxias. As galáxias rádio ativas do tipo Fanaroff-Riley classe II tem pontos quentes no final das estruturas que emitem em rádio sinalizando a existência de jatos de plasma altamente colimados. Mais detalhes sobre os mecanismos de aceleração de partículas neste jatos podem ser encontrados nas referências $(120,121)$. O índice espectral proposto é da ordem de -2 . 


\subsubsection{Centaurus A}

Entre todas as rádio galáxias que de acordo com o modelo acima produzem UHECR, Centaurus $\mathrm{A}$ (Cen $\mathrm{A}$ ) é a que mais contribui com o fluxo na Terra devido à sua proximidade (122). Centaurus A ou NGC 5128 é uma galáxia atípica com morfologia peculiar provavelmente resultante da junção de duas outras galáxias menores $(123,124)$. Distante apenas $3.69 \mathrm{Mpc}$ da Terra, Cen A tem sido exaustivamente estudada. Estima-se que o buraco negro central tenha massa equivalente da ordem de 55 milhões de massas solares.

No tocante a produção de UHECR, os fenômenos centrais são a interação do jato norte com a camada gasosa (125) e a intensa formação estelar neste mesmo local (126). Medidas das vizinhanças dos lóbulos sul e norte indicam a presença de um grande número de camadas estrelares (127) com abundantes quantidades de gases atômicos (128) e moleculares (129). Estudos dos mapas de emissão em rádio mostram que o jato norte encontra essas camadas a distâncias entre 3.5 e 15 kpc do centro $(130,131)$. Simulações mostram que estas colisões indicam a formação de fortes ondas de choque (132).

\subsubsection{UHECR vindos de Cen A}

Cen A é certamente um ambiente astrofísico rico, no entanto, será que Cen $A$ tem as características básicas necessárias para acelerar partículas a $10^{20} \mathrm{eV}$ ?

Utilizando $L_{p c}=6000(125)$ e $B_{\mu G}=3(46)$ temos que $E_{\text {max }} \leq Z \times 10^{19} \mathrm{eV}$, através do critério de Hillas, veja equação 4.0.2. Ou seja, para $Z=1$ (próton), $E_{\max } \leq 10^{19} \mathrm{eV}$ e para $\mathrm{Z}=26$ (Ferro), $E_{\max } \leq 2.6 \times 10^{20} \mathrm{eV}$.

Medidas da luminosidade de Cen $A$ indicam que $L_{\text {jet }} \simeq 10^{43} \mathrm{erg} \mathrm{s}^{-1}(126,133-135)$. Utilizando a equação 4.0.1, temos que $E_{\max } \leq \frac{Z}{f_{\text {int }}} \times 10^{19} \mathrm{eV}$. Determinações precisas de $f_{\text {int }}$ não são possíveis, mas espera-se que seu valor fique entre 0.1 e 1 . Desta forma, o critério de Lovelace leva a uma limitação semelhante ao critério de Hillas para a máxima energia de uma partícula em Cen A. Ambos os critérios apontam para a necessidade de partículas mais pesadas que próton para atingir as máximas energias detectadas $(5,136)$.

O tempo de duração da onda de choque ( $\sim 10^{4}$ anos) (132) é mais do que suficiente para completar a aceleração dado que a escala de tempo de Larmor é da ordem de $\sim 10^{4.3}$ anos. 


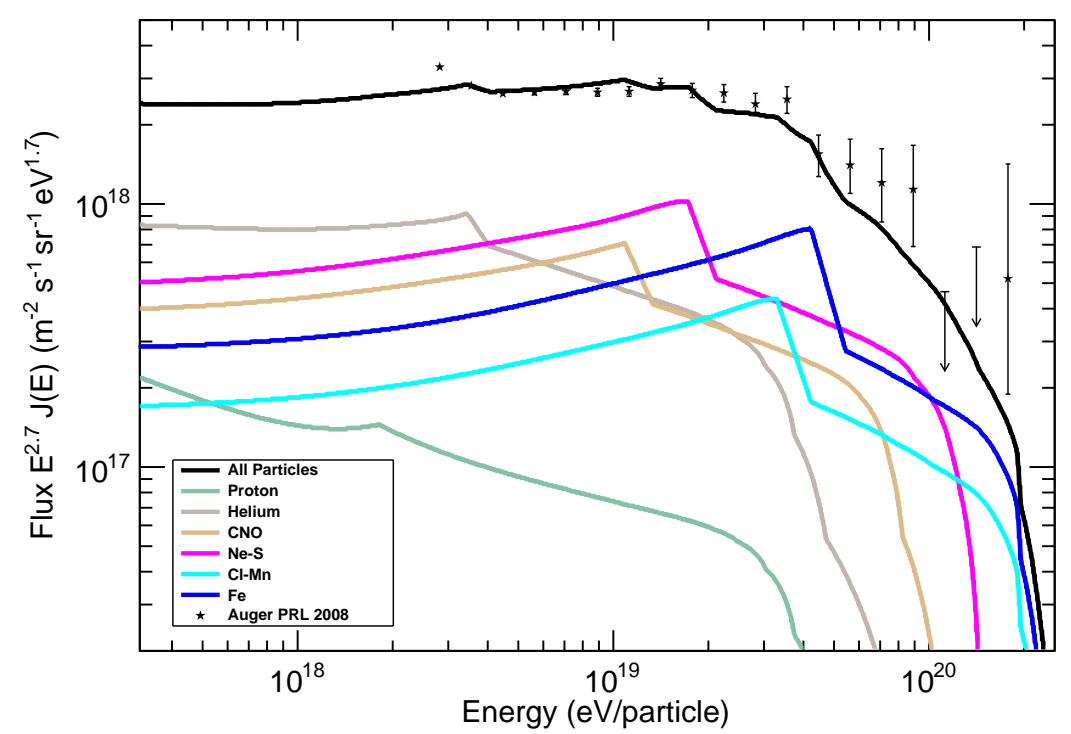

Figura 4.3 - Ajuste da forma do espectro de energia proposto na referência (44) ao dados do Observatório Pierre Auger. Fonte: Figura extraída da referência (46).

Dado a viabilidade do processo e usando as suposições elementares do modelo, realizamos um ajuste do espectro proposto pelo modelo (ver figura 4.2) aos dados medidos pelo Observatório Pierre Auger (5). A ideia central do ajuste era avaliar se a forma do espectro original com suas várias estruturas era capaz de descrever a forma geral do espectro medido. A figura 4.3 mostra o sucesso da proposta tal como publicado na referência (46).

\subsubsection{Via Láctea + Cen A: as fontes de raios cósmicos}

Baseado no sucesso da descrição da forma do espectro de raios cósmicos com o modelo proposto na referência (44), demos o passo seguinte e tentamos descrever com base no mesmo modelo a região de transição entre a predominância galáctica para extragaláctica. Supondo as mesmas fontes galácticas explicadas acima e a mesma forma espectral da figura 4.2, ajustamos o espectro de energia medido pelos experimentos KASCADE e KASCADE-Grande para partículas com energia entre $10^{15} \mathrm{e} 10^{18} \mathrm{eV}$. A figura 4.4 mostra o resultado do ajuste tal como publicado na referência (47).

$\mathrm{O}$ ajuste foi feito primeiramente aos dados dos experimentos KASCADE e KASCADEGrande no qual a abundância relativa de cada elemento é fixada. O espectro ajustado é deslocado em energia e a mesma abundância é mantida. Um novo ajuste é realizado e um 


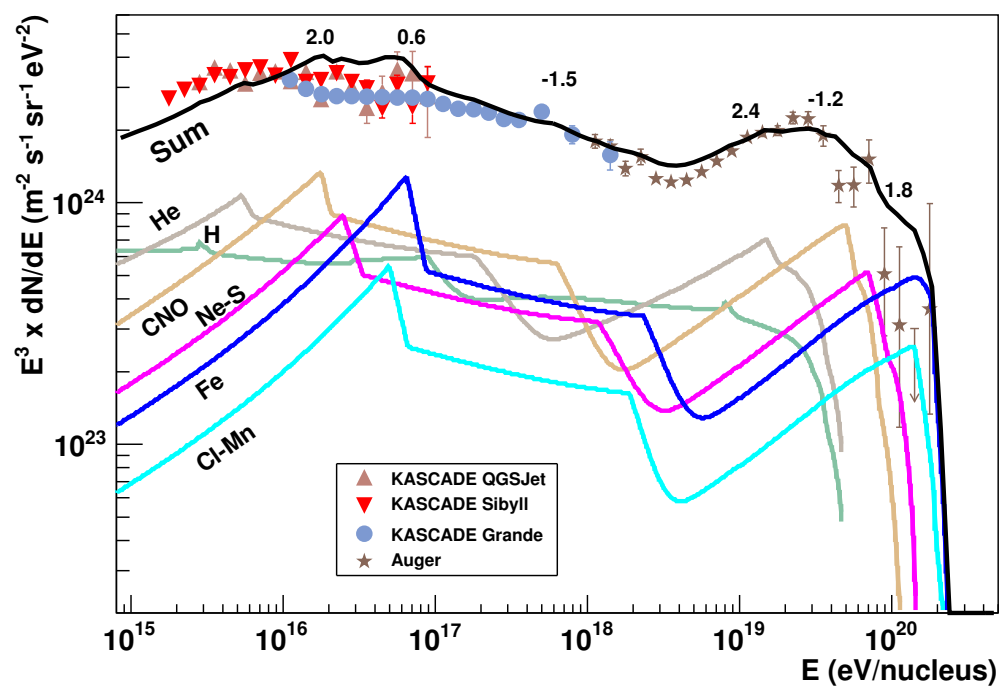

Figura 4.4 - Ajuste do espectro de energia medido pelos Observatórios KASCADE, KASCADEGrande e Pierre Auger. Os números na parte superior da figura mostram um medida do erro do ajuste (Modelo - Dados)/(Erro experimental). Fonte: Figura extraída da referência (47).

fator de deslocamento (3800) em energia é calculado. Para energia acima de $10^{19} \mathrm{eV}$, efeitos de propagação foram levados em conta (ver capítulo 3.)

O espectro de energia de todas a partículas pode ser bem descrito por esse modelo no intervalo de energia de $10^{15}$ a $10^{20} \mathrm{eV}$, entretanto, uma grande degenerescência intrínseca do modelo, relativo as abundância de cada primário não pode ser removida com essa publicação (47). Para atacar este problema, uma terceira publicação foi elaborada (45) na qual o fluxo de cada partícula fora considerado independentemente. Além disso, buscamos ainda estender a validade do modelo para mais baixas energias.

Utilizando dados do experimento AMS-02, ajustamos a normalização do espectro de prótons, e usando os dados dos experimentos KASCADE e KASCADE-Grande do fluxo de prótons ajustamos as quebras no espectro $\left(E_{2}^{H-\text { cutoff }}=1.96 \times 10^{15}\right)$ (veja figura 4.5). Estes ajustes determinam o fluxo relativo de próton para todo o intervalo de energia de $10^{10} \mathrm{a}$ $10^{20} \mathrm{eV}$. Em seguida realizamos ajustes semelhantes para o fluxo de He medido por AMS-02 e CREAM (veja figura 4.6). Infelizmente, o experimento KASCADE e KASCADE-Grande não publicaram uma análise única do fluxo de He de forma que a continuação do ajuste das estruturas do espectro de $\mathrm{He}$ na região de $10^{15} \mathrm{eV}$ não puderam ser feitas. Estudo semeIhante pode ser feito com uma junção de primários de massa intermediaria (veja figura 4.7). E por último ajustamos modelo ao fluxo de núcleo de ferro medido por CREAM, KASCADE e KASCADE-Grande (veja figura 4.8). No caso do ajuste para o fluxo de núcleo de ferro, 


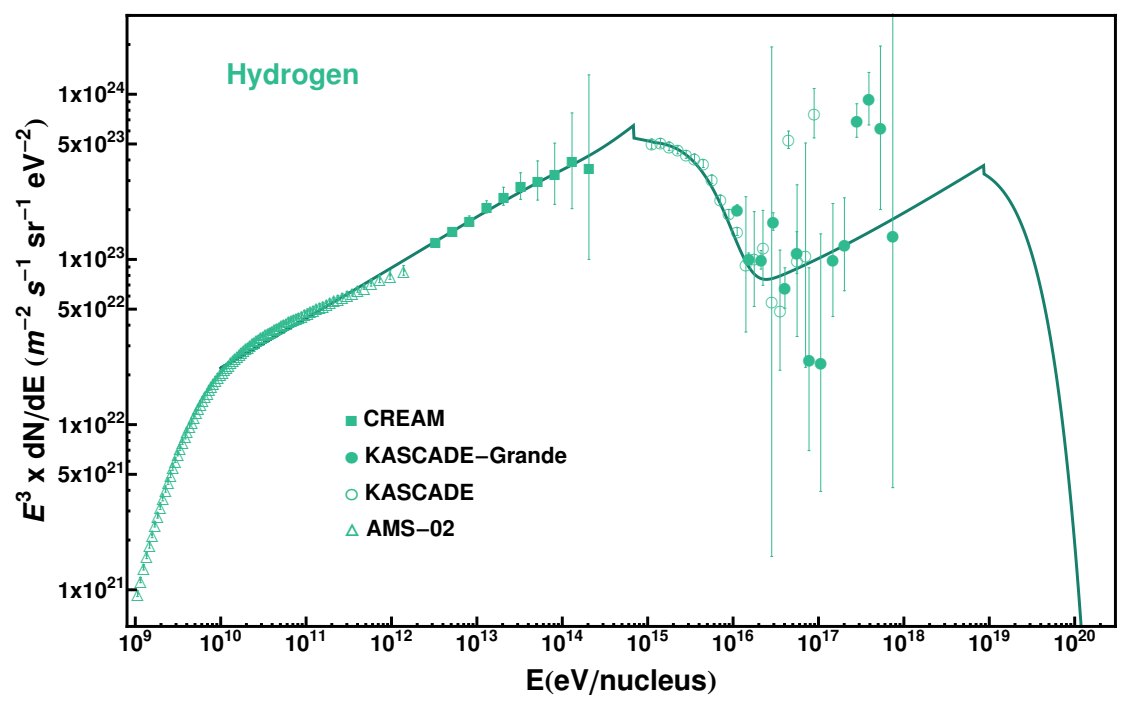

Figura 4.5 - Fluxo de próton $(\mathrm{H})$ em função da energia. Os dados de CREAM $(48,49)$, AMS$02(50), \operatorname{KASCADE}(51,52)$ e $\operatorname{KASCADE}-G r a n d e ~(53,54)$ são mostrados juntamente como modelo considerado. Fonte: Figura extraída da referência (45).

duas hipóteses foram consideradas. Na hipótese original, a quebra no fluxo de ferro seguia a equação: $E_{2}^{\text {cutoff-Fe }}=26 \times E_{2}^{\text {cutoff-H }}$, ou seja, a energia de corte é proporcional à rigidez (Rigidity Dependent). No entanto, a figura 4.8 mostra que essa suposição leva a um ajuste de baixa qualidade dos dados medidos por KASCADE-Grande. Por isso, liberamos esse vínculo e deixamos $E_{2}^{\text {cutoff-Fe }}$ variar no ajuste. O novo valor ajustado foi $E_{2}^{\text {cutoff-Fe }}=2.54 \times 10^{17} \mathrm{eV}$.

Esta nova proposta remove parte de degenerescência do ajuste ao dados ao permitir fixar os parâmetros de alguns tipos de partícula independentemente. Veja na figura 4.9 o resultado final da composição de cada componente. Por último, comparamos os resultados do ajuste à medidas de composição feitas pelo Observatório Pierre Auger (figuras 4.10 e 4.11. Esta análise mostra também a necessidade de uma componente extra de núcleos de ferro, ou outro tipo de partícula pesada, para descrever a transição entre $10^{16}$ e $10^{18} \mathrm{eV}$. No entanto, a análise dos parâmetros sensíveis a composição (figuras 4.10 e 4.11) revela que as atuais propostas já são em média mais pesadas do que os dados tornando explícito a dificuldade de compatibilizar o espectro medido com a composição no contexto deste modelo. 


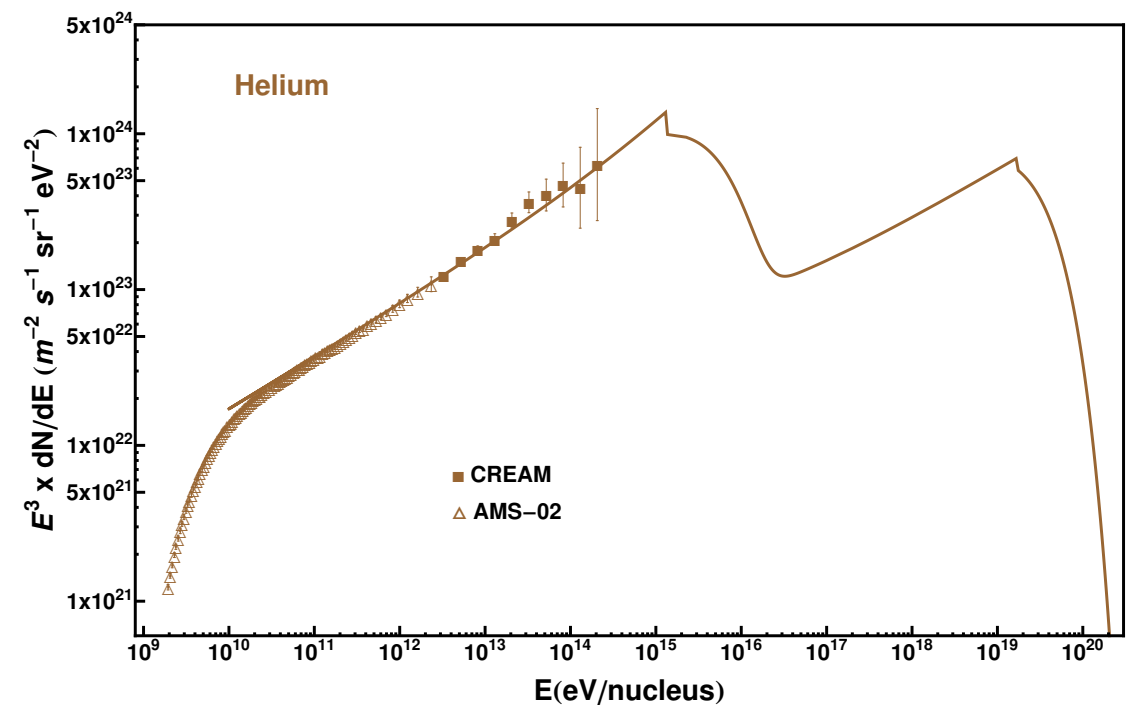

Figura 4.6 - Fluxo de núcleos de hélio em função da energia. Os dados de $\operatorname{CREAM}(48,49)$ e AMS-02 (50) são mostrados juntamente como modelo considerado. Todas as quebras e cortes de energia seguem o modelo de dependência com a rigidez. Fonte: Figura extraída da referência (45).

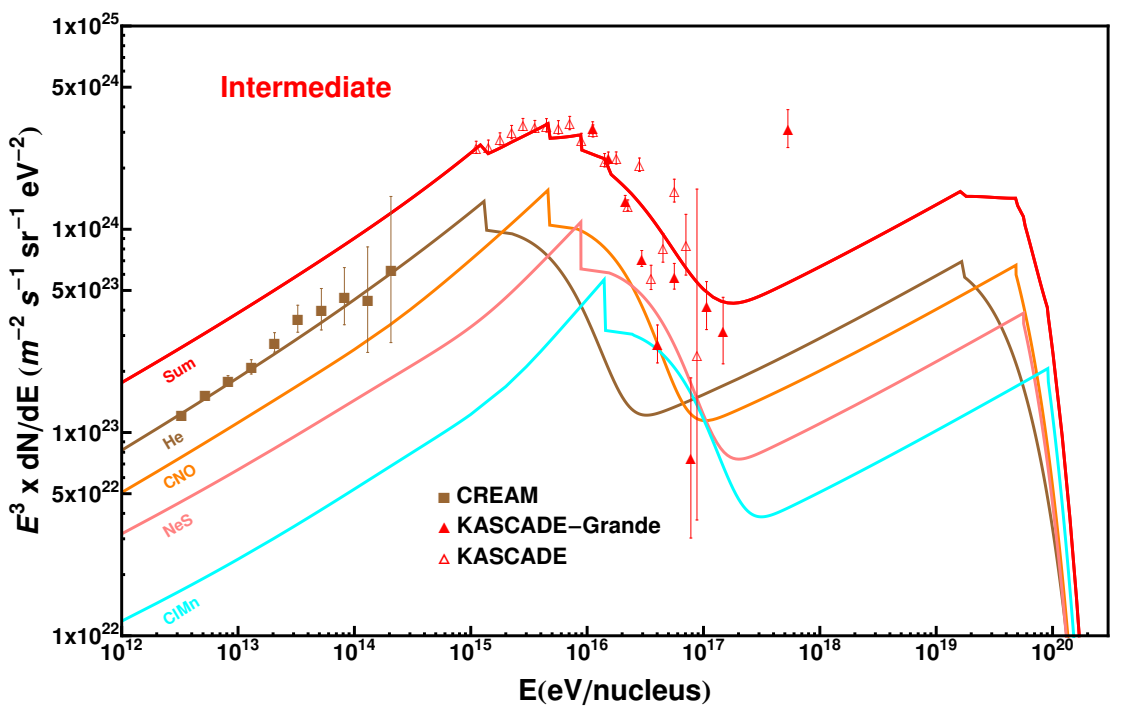

Figura 4.7 - Fluxo de núcleos com massa intermediária ( $\mathrm{He}, \mathrm{CNO}, \mathrm{NeS}$ and $\mathrm{CIMn}$ ) em função da energia. Dados de CREAM $(48,49), \operatorname{KASCADE}(51,52)$ e KASCADE-Grande $(53,54)$ são mostrados juntamente como modelo considerado. Todas as quebras e cortes de energia seguem o modelo de dependência com a rigidez. Fonte: Figura extraída da referência (45). 


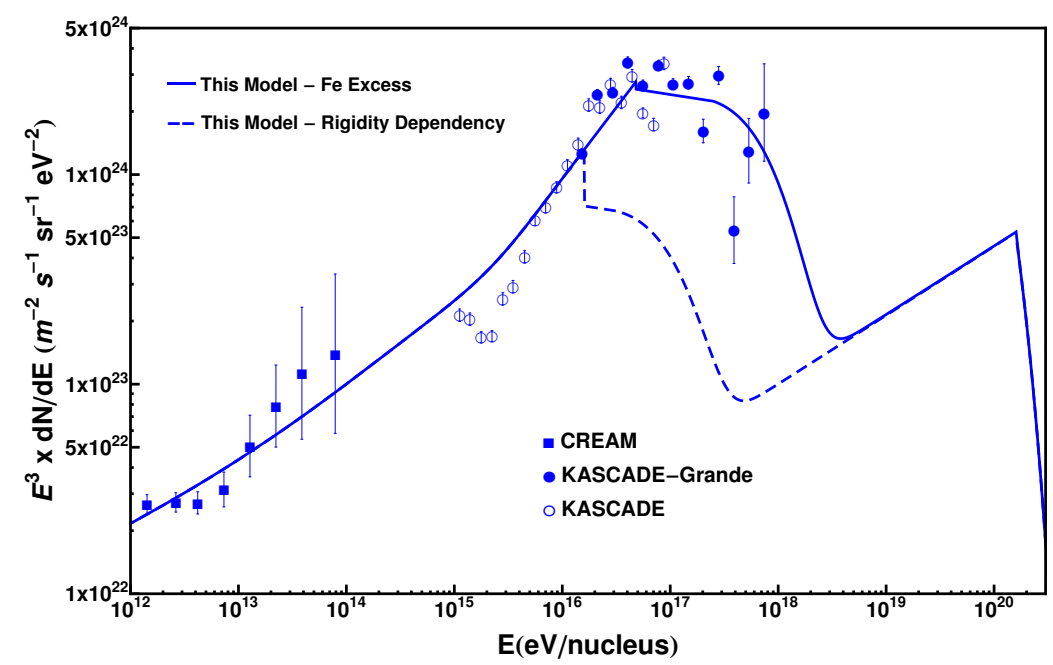

Figura 4.8 - Fluxo de núcleos de ferro em função da energia. Dados de CREAM $(48,49)$, KASCADE $(51,52)$ e KASCADE-Grande $(53,54)$ são mostrados juntamente como modelo considerado. "This model - Rigidity Dependency" é usado para identificar a hipótese na qual $E_{2}^{\text {cutoff }-e}=Z \times E_{2}^{\text {cutoff }-H}$ para todos os elementos. "This model - Fe excess" é usado para identificar a hipótese na qual $E_{2}^{\text {cutoff }-e}=Z \times E_{2}^{\text {cutoff-H }}$ para todos os elementos exceto Fe. Fonte: Figura extraída da referência (45).

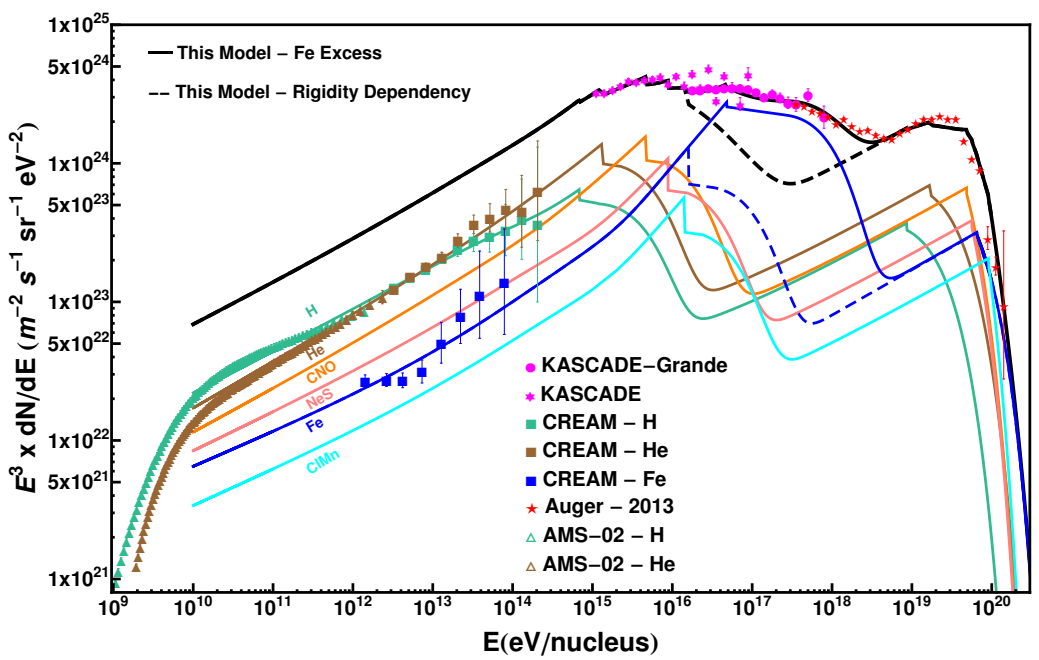

Figura 4.9 - Fluxo de todas as partículas em função da energia. Dados de CREAM (48, 49), AMS-02 (50), KASCADE (51, 52), KASCADE-Grande (53, 54) e Auger (5) são mostrados juntamente como modelo considerado. A extrapolação para altas energias foi feita usando o deslocamento proposto na referência (47). "This model - Rigidity Dependency" é usado para identificar a hipótese na qual $E_{2}^{\text {cutoff }-e}=Z \times E_{2}^{\text {cutoff } f-H}$ para todos os elementos. "This model - Fe excess" é usado para identificar a hipótese na qual $E_{2}^{\text {cutoff }-e}=Z \times E_{2}^{\text {cutoff }-H}$ para todos os elementos exceto Fe. Fonte: Figura extraída da referência (45). 


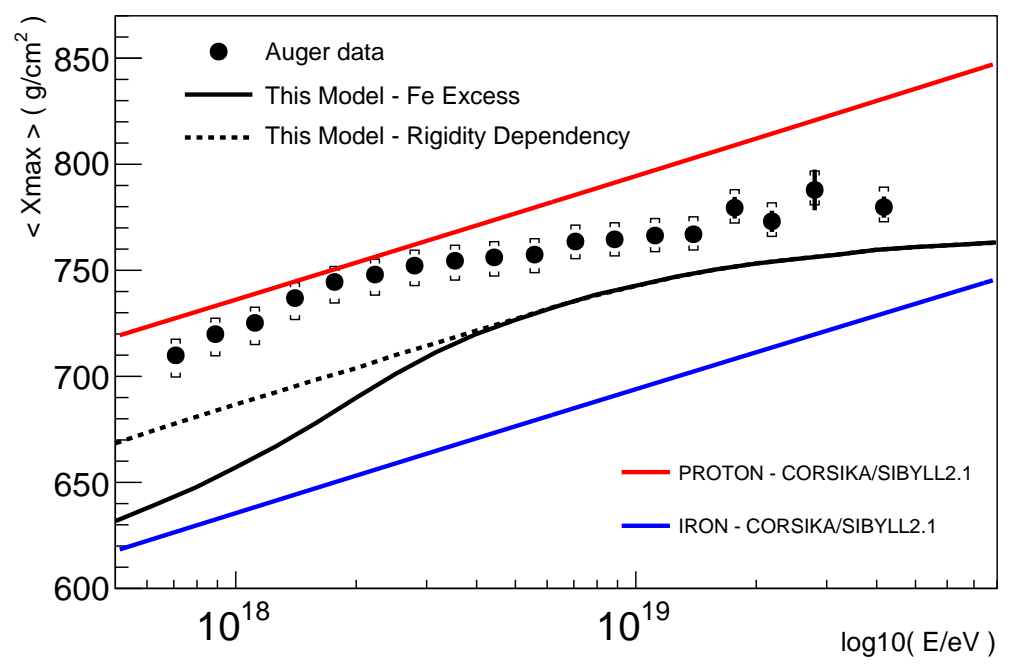

Figura 4.10 - Média da profundidade do máximo do chuveiro $\left(\left\langle X_{\max }\right\rangle\right)$ em função da energia. Dados do Observatório Pierre Auger são mostrados (55). "This model - Rigidity Dependency" é usado para identificar a hipótese na qual $E_{2}^{\text {cutoff }-e}=Z \times E_{2}^{\text {cutoff }-H}$ para todos os elementos. "This model - Fe excess" é usado para identificar a hipótese na qual $E_{2}^{\text {cutoff-e }}=Z \times E_{2}^{\text {cutoff }-H}$ para todos os elementos exceto Fe. Fonte: Figura extraída da referência (45).

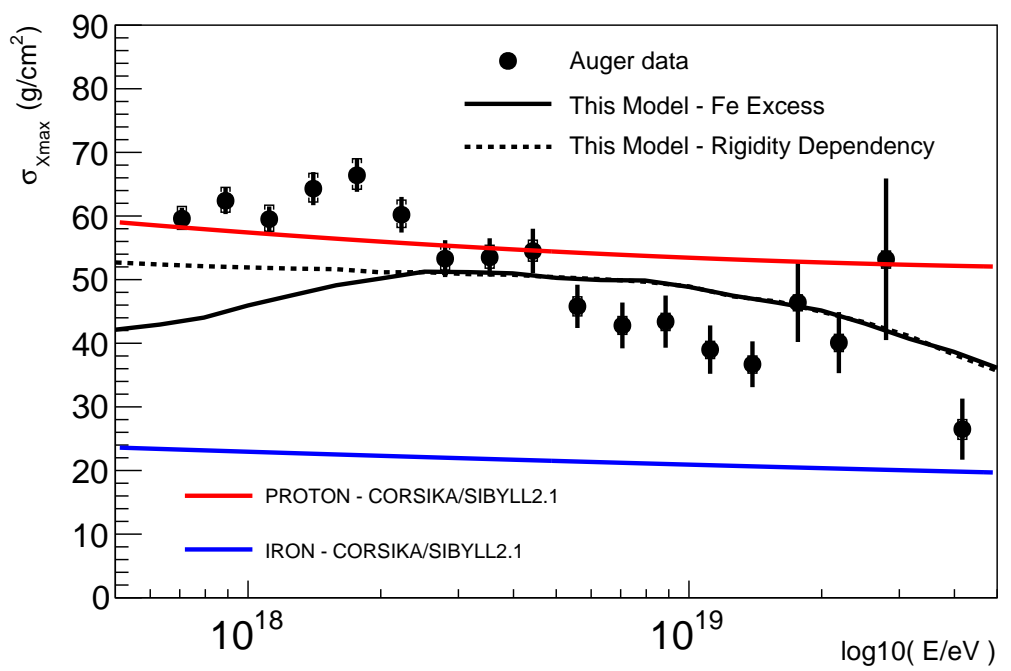

Figura 4.11 - Dispersão da profundidade do máximo do chuveiro $\left(\operatorname{RMS}\left(X_{\max }\right)\right)$ em função da energia. Dados do Observatório Pierre Auger são mostrados (55). "This model - Rigidity Dependency" é usado para identificar a hipótese na qual $E_{2}^{\text {cutof } f-e}=Z \times E_{2}^{\text {cutoff }-H}$ para todos os elementos. "This model - Fe excess" é usado para identificar a hipótese na qual $E_{2}^{\text {cutoff-e }}=Z \times E_{2}^{\text {cutoff-H }}$ para todos os elementos exceto Fe. Fonte: Figura extraída da referência (45). 


\section{Capítulo 5}

\section{Perspectivas futuras}

Apresento neste capítulo um breve comentário sobre os resultados descritos ao longo desta tese e detalho as perspectivas futuras de duas atividades experimentais.

No capítulo 4, mostramos o estudo detalhado de um modelo de aceleração de partículas (44) que propõem a descrição do espectro de raios cósmicos com energia entre $10^{10}$ e $10^{20} \mathrm{eV}$. O estudo foi realizado em três fases que resultaram em três publicações (45-47). O intuito do trabalho foi sempre de reduzir as liberdades do modelo utilizando dados medidos pelos melhores observatório. Duas principais conclusões desta série de trabalho podem ser feitas. Primeiramente, mostramos que a mesma forma espectral pode ser usada para descrever duas regiões distintas do espectro $10^{10}$ a $10^{16} \mathrm{eV}$ e $10^{18}$ a $10^{20} \mathrm{eV}$, correspondendo a raios cósmicos galáticos e extragaláticos, respectivamente. A região de transição $10^{16}$ a $10^{18} \mathrm{eV}$ foi também estudada e mais uma vez a necessidade de melhores dados foi discutida. Em seguida, podemos concluir que a variação da contribuição relativa de cada tipo de primário ao fluxo total é muito pequena ao longo de todo o intervalo de energia considerado $10^{10}$ a $10^{20} \mathrm{eV}$ o que não deixa de ser um fato muito surpreendente. Nas regiões de mais altas energias $E>10^{18} \mathrm{eV}$, a contribuição de cada tipo de partícula é modulada por efeitos de propagação.

Apesar dos nossos esforços de reduzir a degenerescência do modelo original e de limitar os parâmetros propostos, ainda restaram muitos graus de liberdade. Para restringi-los todos, precisaríamos de medidas muito mais precisas do espectro e sobretudo de medidas do fluxo de cada tipo de partícula. Essas limitações experimentais restringem as possibilidades de continuar desenvolvendo o modelo para além das publicações já realizadas.

No capítulo 3, discutimos a geração de raios gamas com energia entre GeV e TeV a partir de um fluxo inicial de raios cósmicos com energia acima de EeV. Propusemos um modelo que relaciona o limite da integral do fluxo de gamas ( $\mathrm{GeV}-\mathrm{TeV}$ ) com a luminosidade de raios cósmicos (41) e exploramos essa proposta utilizando fontes medidas pelos Observatórios FERMI-LAT, H.E.S.S., VERITAS e MAGIC (42). Os cálculos impõem limites no fluxo de raios cósmicos de fontes pontuais, resultado esse impossível até o presente de ser realizado com base nos dados de observatório de raios cósmicos. O limite imposto pelos nossos cálculos não é restritivo o suficiente para excluir nenhum modelo de aceleração ou algum tipo de fonte. 
No entanto, a construção do Observatório CTA irá abaixar em uma ordem de grandeza o limiar do fluxo de raios gamas com energia entre $\mathrm{GeV}$ e $\mathrm{TeV}$. Com base nas medidas realizadas pelo CTA, seremos capazes de testar os modelos de aceleração usando nossa proposta.

Outras possibilidades de estudo restam para serem feitas com base nesta proposta. $O$ efeito do campo magnético extragalático e galático foram desprezados neste estudo. Por um lado, o campo magnético diminuirá o sinal de gamas secundários porque o caminho do raio cósmico primário será desviado e o raio gama gerado não necessariamente atingirá a Terra. Por outro lado, o campo magnético aumenta o percurso do raio cósmico aumentando a probabilidade de interação deste com o meio e consequentemente aumentando o fluxo de raios gama ( $\mathrm{GeV}-\mathrm{TeV})$. O balanço entre os dois efeitos precisa ser estudado.

Da mesma forma, seria muito interessante analisar a possibilidade de utilizarmos fontes com espectro de energia medido e avaliar qual a possível contribuição de gamas secundários. Ou ao revés, subtrair um espectro primário de fótons de uma medida experimental para calcular o espectro secundário e utilizá-lo para impor um limite no fluxo de UHECR.

Do ponto de vista experimental, nossas perspectivas de trabalho futuro estão relacionas com AugerPrime e CTA que serão descritos abaixo.

\subsection{AugerPrime}

AugerPrime é uma proposta de extensão da configuração original do Observatório Pierre Auger com o intuito de atacar as perguntas ainda em aberto sobre a produção de UHECR. As três motivações principais para a extensão são:

- Medir a composição de massas dos raios cósmicos de energia superior a $10^{19} \mathrm{eV}$ e com isso resolver se a supressão do espectro é decorrente do efeito da interação das partículas com a radiação de fundo ou se é o limite de aceleração da fonte;

- Selecionar uma amostra de $10 \%$ de eventos de prótons com energia acima de $10^{19.5} \mathrm{eV}$ para ser capaz de identificar as fontes pontuais abrindo as janelas da astronomia de prótons;

- Estudar os chuveiros atmosféricos extenso e extrair informações sobre as interações hadrônicas em regiões de energia inacessíveis para aceleradores de partículas. 
Estes objetivos serão alcançando com a operação continuada do Observatório com seus detectores atualmente instalados até 2024 e com a instalação de novos detectores dedicados para medir a componente muônica dos chuveiros atmosféricos.

Múons são os principais mensageiros das interações hadrônicas em chuveiros atmosféricos extensos. Desta forma, medir múons com grande precisão irá ao mesmo tempo, permitir estudos de composição para energias acima de $10^{19} \mathrm{eV}$, estudar o chuveiro atmosférico extenso e melhorar as técnicas de reconstrução de energia e direção atualmente empregadas.

Dois tipos de detectores de múons serão instalados nos próximos anos no Observatório Pierre Auger. Cintiladores de plásticos serão instalados sobre os tanques Čerenkov com o intuito de fazer uma medida dupla das partículas. Como a resposta de cada detector é diferente a componente muônica do chuveiro, a razão do sinal dos detectores leva à uma reconstrução precisa do número de múons. Ao mesmo tempo, uma região pequena do Observatório (23.5 $\mathrm{km}^{2}$ ) será instrumentada com cintiladores enterrados no solo. O solo atenua a componente eletromagnética dos chuveiros deixando, após alguns metros, apenas múons como sinal. Esta medida direta de múons será usada para calibrar a técnica de determinação de múons com cintiladores instalados sobre os tanques Čerenkov.

Estamos envolvidos de duas maneiras nesta proposta. Do ponto de vista experimental, implementaremos uma técnica alternativa de calibração do sinal de múons baseado em detectores RPC (137). Uma colaboração luso-brasileira instalará nos próximos dois anos 24 detectores no sítio do Observatório Pierre Auger e testará sua eficácia e resolução. Não obstante, estamos desenvolvendo técnicas de análise do sinal de múons bem como a sua utilização para separação de modelos astrofísicos e de interação hadrônica.

\subsection{CTA - Cherenkov Telescope Array}

A detecção de raios gama utilizado telescópios de luz Cherenkov é uma técnica consolidada que tem contribuído significativamente para o avanço do nosso entendimento de vários fenômenos em astrofísica de partículas. A história de experimentos de raios gama terrestres pode ser dividida em 3 fases: pioneira, estereoscópica e de grandes conjuntos de telescópios. Atualmente, estamos vivendo esta última fase, com a proposta do experimento Cherenkov Telescope Array (CTA) por um consórcio internacional. Inicialmente as comunidades europeia e estadunidense propuseram dois experimentos independentes, respectivamente CTA e AGIS. No entanto, uma junção dos dois experimentos em torno da proposta europeia concretizou-se 


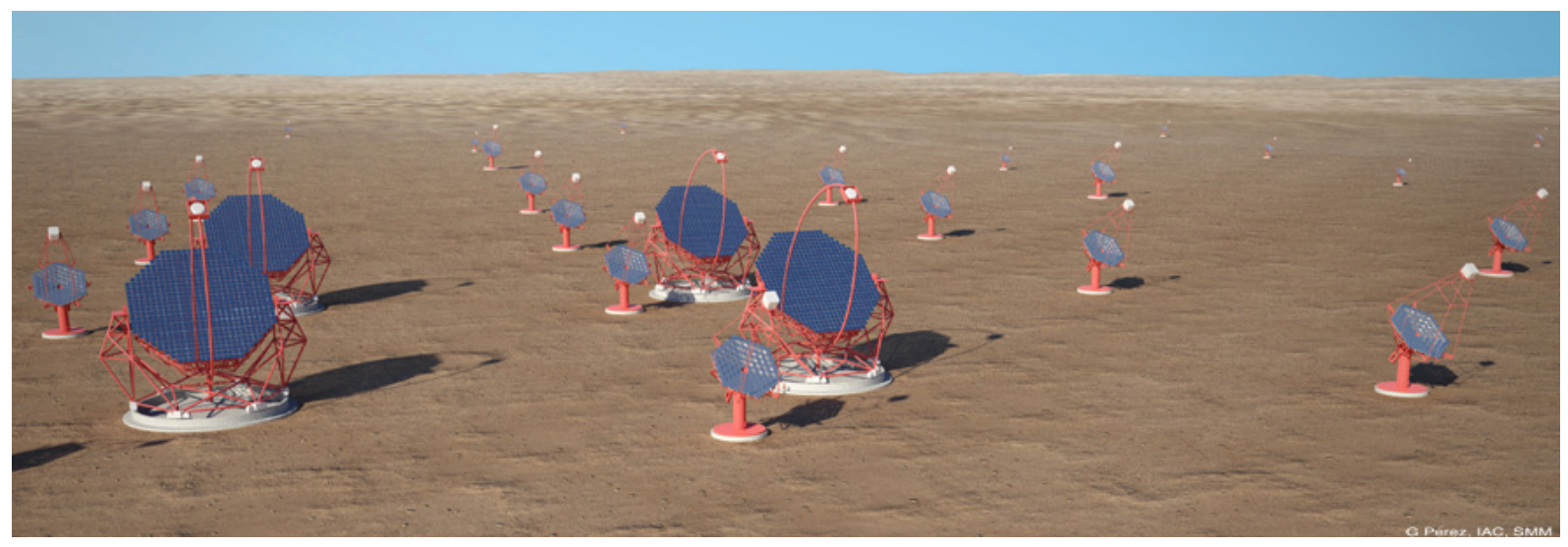

Figura 5.1 - Visão artística do Observatório CTA. Fonte: Figura produzida pela Colaboração CTA disponível somente para seus membros.

em Maio de 2010.

O experimento pioneiro Whipple (138) começou suas operações em 1968 e demonstrou a eficiência da técnica. A fase estereoscópica, a segunda fase mencionada acima, iniciou-se por volta do ano 2000. Os experimentos H.E.S.S. (139), CANGAROO(140), VERITAS(141) e MAGIC (142) mostraram como que a operação de conjuntos de telescópio poderiam aumentar a sensibilidade, melhorar a eficiência de seleção de raios gamas contra o ruído de outras partículas e diminuir o erro angular no posicionamento da fonte. Estes avanços permitiram o estudo de vários mecanismos inacessíveis por outras técnicas e a detecção de novas fontes com emissão na região de TeV. O funcionamento destes experimentos na última década causou uma revolução na compreensão de vários mecanismos, o que pode ser constatado por uma explosão no número de publicações nesta área.

O sucesso da fase estereoscópica levou à proposta de construção do CTA(143) que será um arranjo de telescópios de diferentes tamanhos e espaçamento que pretende estudar o intervalo de energia entre $10 \mathrm{GeV}$ e $100 \mathrm{TeV}$ com resolução angular e limiar de detecção sem precedentes. A configuração final do tamanho e arranjo de telescópios ainda é objeto de estudo. A figura 5.1 mostra uma visão artística dos telescópios.

Neste contexto, espera-se que o CTA possa melhorar significativamente nossa compreensão de uma imensa gama de mecanismos astrofísicos acontecendo em objetos galáticos e extragaláticos. A figura 5.2 ilustra de forma sucinta e clara a potencialidade do CTA. Esta figura mostra o fluxo previsto de fótons para os principais tipos de objetos conhecidos. A linha sólida mostra a sensitividade dos telescópios (H.E.S.S.) em funcionamento na atualidade. A linha tracejada mostra a sensitividade prevista pra o CTA. Fica evidente que não é possível medir a maior parte da emissão gama dos objetos conhecidos com os instrumentos atualmente em funcionamento. 


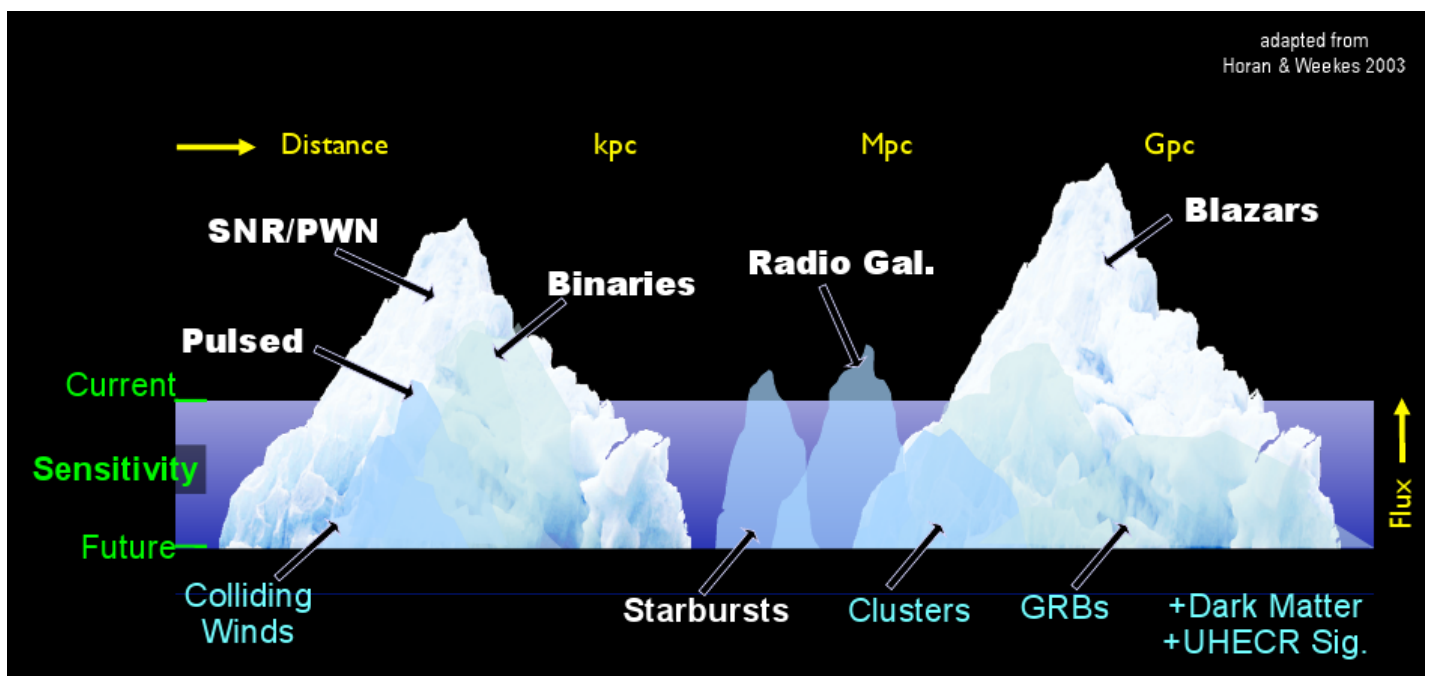

Figura 5.2 - llustração das potencialidades do CTA. A figura mostra o fluxo de raios gamas em função da distância da fonte. Fonte: Ilustração adaptada da referência (56).

O escopo científico do CTA abrange várias questões crucias da astrofísica de partículas e física de altas energias, tais como, a origem dos raios cósmicos, a física de objetos compactos, pulsares e buracos negros, estrelas binárias, supernovas, nuvens moleculares gigantes, matéria escura e os Gamma-ray bursts (GRBs). Esta área de pesquisa abrange a exploração do Universo não-térmico. A maior parte da astronomia convencional concentra-se no estudo da radiação emitida por estrelas produzida predominantemente por processos térmicos ou colisionais. No entanto, sabemos que algumas formas de radiação independem da temperatura do gás e exigem a ação de um processo coletivo de concentração de energia sobre uma pequena população de partículas. A maior evidência destes processos é sem dúvida o espectro de energia de raios cósmicos que se estende até $10^{20} \mathrm{eV}$ e não pode ser reduzido a um processo térmico.

Nosso interesse na física a ser realizada pelos CTA está focado nos seguintes assunto: a) nova física: matéria escura e quebra de invariância de Lorentz, b) propagação: relação entre UHECR e gamas (GeV-TeV), c) chuveiros atmosférico extenso e configuração dos telescópios e d) instrumentação. Recentemente, temos estudados estes assuntos e nos envolvemos com a construção do telescópio de tamanho médio mostrado na figura 5.3. A parte cinza da estrutura foi elaborada, desenvolvida e construída por nós em colaboração com a empresa Orbital Engenharia. 


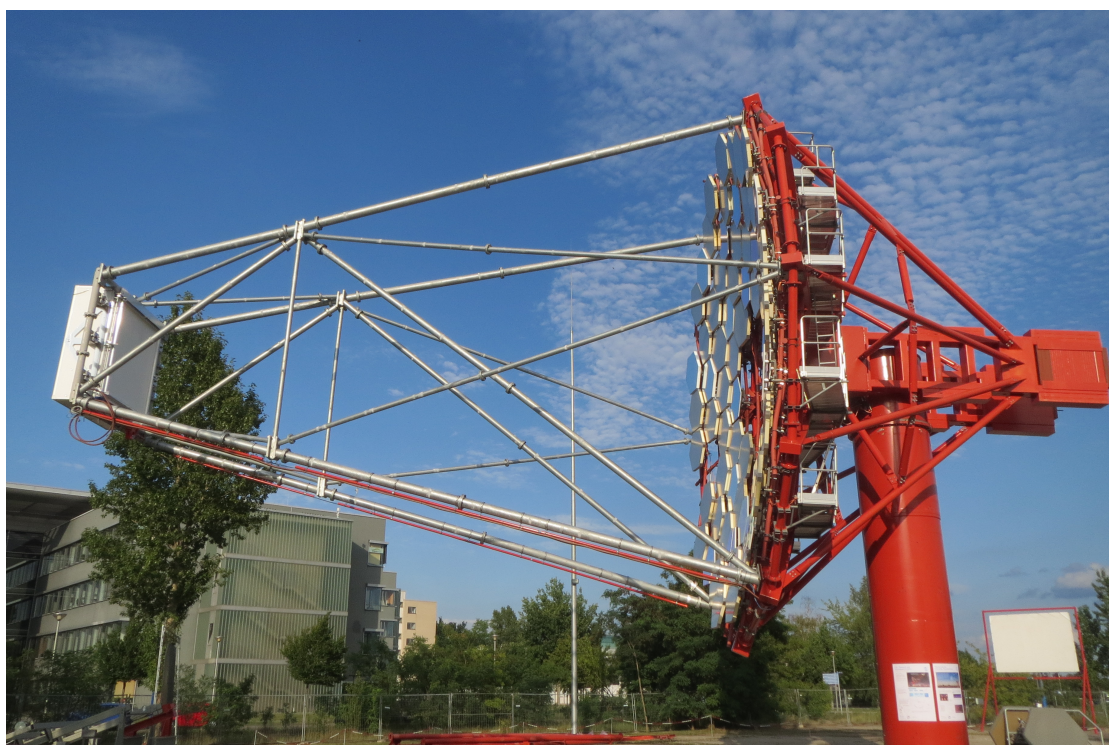

Figura 5.3 - Protótipo do telescópio médio do CTA instalado em Berlim. A parte cinza foi elaborada, desenvolvida e construída por nós em colaboração com a empresa Orbital Engenharia. Fonte: Foto tirada por Ronald Shellard. 


\section{REFERÊNCIAS}

1 GAISSER, T. K.; HILLAS, A. M. Reliability of The Method of Constant Intensity Cuts For Reconstructing the Average Development of Vertical Showers. In: $17^{\mathrm{ND}}$ Int. Cosmic Ray Conf. Plovdiv (Bulgaria): , c1977. p. 353-356.

2 HECK, D.; KNAPP, J.; CAPDEVIELLE, J. N.; SCHATZ, G.; THOUW, T. A Monte-Carlo Code to Simulate Extensive Air Showers - Report FZKA 6019. Technical report, Forschungszentrum Karlsruhe, www-ik.fzk.de/ heck/corsika, 1998.

3 FLETCHER, R. S.; GAISSER, T. K.; LIPARI, P.; STANEV, T. Sibyll: An event generator for simulation of high energy cosmic ray cascades. Physical Review D, v. 50, n. 9, p. 5710-5731, Nov. 1994.

4 TODERO PEIXOTO, C. J.; DE SOUZA, V.; ALFREDO BELLIDO, J. Comparison of the moments of the distribution predicted by different cosmic ray shower simulation models. Astroparticle Physics, v. 47, p. 18-30, July 2013.

5 The Pierre Auger Collaboration. Observation of the suppression of the flux of cosmic rays

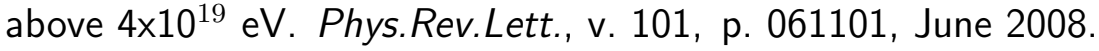

6 The Pierre Auger Collaboration et al. SEARCHES FOR ANISOTROPIES IN THE ARRIVAL DIRECTIONS OF THE HIGHEST ENERGY COSMIC RAYS DETECTED BY THE PIERRE AUGER OBSERVATORY. The Astrophysical Journal, v. 804, n. 1, p. 15, Apr. 2015.

7 The Pierre Auger Collaboration et al. Depth of maximum of air-shower profiles at the Pierre Auger Observatory. I. Measurements at energies above $10^{17.8} \mathrm{eV}$. Physical Review D, v. 90, n. 12, Dec. 2014.

8 Raul Ribeiro Prado. Estudo da composição de raios cósmicos de altas energias através da análise de dados medidos pelo Observatório Pierre Auger. 2014. Tese (Doutorado em Física) - Universidade de São Paulo, São Carlos, 2014.

9 DOLE, H.; LAGACHE, G.; PUGET, J.-L.; CAPUTI, K. I.; FERNáNDEZ-CONDE, N.; LE FLOC'H, E.; PAPOVICH, C.; PéREZ-GONZáLEZ, P. G.; RIEKE, G. H.; BLAYLOCK, M. The cosmic infrared background resolved by Spitzer. Astronomy and Astrophysics, Berlin, v. 451, n. 2, p. 417-429, May 2006.

10 DE ANGELIS, A.; GALANTI, G.; RONCADELLI, M. Transparency of the Universe to gamma rays. Monthly Notices of the Royal Astronomical Society, London, v. 432, n. 4, p. 3245-3249, July 2013. arXiv: 1302.6460. 
11 CHARY, R.; CASERTANO, S.; DICKINSON, M. E.; FERGUSON, H. C.; EISENHARDT, P. R. M.; ELBAZ, D.; GROGIN, N. A.; MOUSTAKAS, L. A.; REACH, W. T.; YAN, H. The Nature of Faint 24 Micron Sources Seen in Spitzer Space Telescope Observations of ELAIS N1. The Astrophysical Journal Supplement Series, v. 154, n. 1, p. 80-86, Sept. 2004.

12 GARDNER, J. P.; BROWN, T. M.; FERGUSON, H. C. Ultraviolet Galaxy Counts from Space Telescope Imaging Spectrograph Observations of the Hubble Deep Fields. The Astrophysical Journal, v. 542, n. 2, p. L79-L82, Oct. 2000.

13 HAUSER, M. G.; DWEK, E. The Cosmic Infrared Background: Measurements and Implications. Annual Review of Astronomy and Astrophysics, v. 39, n. 1, p. 249-307, Sept. 2001. arXiv: astro-ph/0105539.

14 MATSUURA, S. et al. DETECTION OF THE COSMIC FAR-INFRARED BACKGROUND IN AKARI DEEP FIELD SOUTH. The Astrophysical Journal, v. 737, n. 1, p. 2, Aug. 2011.

15 KEENAN, R. C.; BARGER, A. J.; COWIE, L. L.; WANG, W.-H. THE RESOLVED NEARINFRARED EXTRAGALACTIC BACKGROUND. The Astrophysical Journal, v. 723, n. 1, p. 40-46, Nov. 2010.

16 SCHLEGEL, D. J.; FINKBEINER, D. P.; DAVIS, M. Maps of Dust Infrared Emission for Use in Estimation of Reddening and Cosmic Microwave Background Radiation Foregrounds. The Astrophysical Journal, v. 500, n. 2, p. 525-553, June 1998.

17 FINKBEINER, D. P.; DAVIS, M.; SCHLEGEL, D. J. Detection of a Far-Infrared Excess with DIRBE at 60 and 100 Microns. The Astrophysical Journal, v. 544, n. 1, p. 81-97, Nov. 2000.

18 BERNSTEIN, R. A. The Optical Extragalactic Background Light: Revisions and Further Comments. The Astrophysical Journal, v. 666, n. 2, p. 663-673, Sept. 2007.

19 FIXSEN, D. J. THE TEMPERATURE OF THE COSMIC MICROWAVE BACKGROUND. The Astrophysical Journal, v. 707, n. 2, p. 916-920, Dec. 2009.

$20 \mathrm{XU}, \mathrm{C}$. K. et al. Number Counts of GALEX Sources in Far-Ultraviolet $(1530 \AA)$ and Near-Ultraviolet (2310 А̊) Bands. The Astrophysical Journal, v. 619, n. 1, p. L11-L14, Jan. 2005.

21 FRAYER, D. T.; HUYNH, M. T.; CHARY, R.; DICKINSON, M.; ELBAZ, D.; FADDA, D.; SURACE, J. A.; TEPLITZ, H. I.; YAN, L.; MOBASHER, B. S p i t zer 70 Micron Source Counts in GOODS-North. The Astrophysical Journal, v. 647, n. 1, p. L9-L12, Aug. 2006.

22 BéTHERMIN, M.; DOLE, H.; BEELEN, A.; AUSSEL, H. Spitzer deep and wide legacy mid- and far-infrared number counts and lower limits of cosmic infrared background. Astronomy and Astrophysics, Berlin, v. 512, p. A78, Mar. 2010. 
23 HAUSER, M. G. et al. The COBE Diffuse Infrared Background Experiment Search for the Cosmic Infrared Background: I. Limits and Detections. The Astrophysical Journal, v. 508, n. 1, p. 25-43, Nov. 1998. arXiv: astro-ph/9806167.

24 LEVENSON, L. R.; WRIGHT, E. L. Probing the $3.6 \mu \mathrm{m}$ CIRB with Spitzer in Three DIRBE Dark Spots. The Astrophysical Journal, v. 683, n. 2, p. 585-596, Aug. 2008.

25 BERTA, S. et al. Dissecting the cosmic infra-red background with Herschel /PEP. Astronomy and Astrophysics, Berlin, v. 518, p. L30, July 2010.

26 MADAU, P.; POZZETTI, L. Deep galaxy counts, extragalactic background light and the stellar baryon budget. Monthly Notices of the Royal Astronomical Society, London, v. 312, n. 2, p. L9-L15, Feb. 2000.

27 FAZIO, G. G. et al. The Infrared Array Camera (IRAC) for the Spitzer Space Telescope. The Astrophysical Journal Supplement Series, v. 154, n. 1, p. 10-17, Sept. 2004.

28 METCALFE, L.; KNEIB, J.-P.; MCBREEN, B.; ALTIERI, B.; BIVIANO, A.; DELANEY, M.; ELBAZ, D.; KESSLER, M. F.; LEECH, K.; OKUMURA, K.; OTT, S.; PEREZMARTINEZ, R.; SANCHEZ-FERNANDEZ, C.; SCHULZ, B. An ISOCAM survey through gravitationally lensing galaxy clusters: I. Source lists and source counts for A370, A2218 and A2390. Astronomy and Astrophysics, Berlin, v. 407, n. 3, p. 791-822, Sept. 2003.

29 CAMBRESY, L.; REACH, W. T.; BEICHMAN, C. A.; JARRETT, T. H. The Cosmic Infrared Background at 1.25 and 2.2 Microns Using DIRBE and 2mass: A Contribution Not Due to Galaxies? The Astrophysical Journal, v. 555, n. 2, p. 563-571, July 2001.

30 Rita de Cássia dos Anjos. Propagação de raios cósmicos extragaláticos. 2014. Tese (Doutorado em Física) - Universidade de São Paulo, São Carlos, 2014.

31 MALKAN, M. A.; STECKER, F. W. An Empirically Based Model for Predicting Infrared Luminosity Functions, Deep Infrared Galaxy Counts, and the Diffuse Infrared Background. The Astrophysical Journal, v. 555, n. 2, p. 641-649, July 2001.

32 GILMORE, R. C.; SOMERVILLE, R. S.; PRIMACK, J. R.; DOMíNGUEZ, A. Semi-analytic modelling of the extragalactic background light and consequences for extragalactic gammaray spectra: Modelling of the EBL and gamma-ray spectra. Monthly Notices of the Royal Astronomical Society, London, v. 422, n. 4, p. 3189-3207, June 2012.

33 AHARONIAN, F. et al. A low level of extragalactic background light as revealed by $\gamma$-rays from blazars. Nature, v. 440, n. 7087, p. 1018-1021, Apr. 2006.

34 MAZIN, D.; RAUE, M.; BEHERA, B.; INOUE, S.; INOUE, Y.; NAKAMORI, T.; TOTANI, T.; CONSORTIUM, F. T. C. Potential of EBL and cosmology studies with the Cherenkov Telescope Array. Astroparticle Physics, v. 43, p. 241-251, Mar. 2013. arXiv: 1303.7124. 
35 The MAGIC Collaboration et al. Very-High-Energy Gamma Rays from a Distant Quasar: How Transparent Is the Universe? Science, v. 320, n. 5884, p. 1752-1754, June 2008.

36 AHARONIAN, F. et al. Observations of H1426+428 with HEGRA: Observations in 2002 and reanalysis of 1999\&amp;2000 data. Astronomy and Astrophysics, Berlin, v. 403, n. 2, p. 523-528, May 2003.

37 FRANCESCHINI, A.; AUSSEL, H.; CESARSKY, C. J.; ELBAZ, D.; FADDA, D. A longwavelength view on galaxy evolution from deep surveys by the Infrared Space Observatory. Astronomy and Astrophysics, Berlin, v. 378, n. 1, p. 1-29, Oct. 2001.

38 FRANCESCHINI, A.; RODIGHIERO, G.; VACCARI, M. Extragalactic optical-infrared background radiation, its time evolution and the cosmic photon-photon opacity. Astronomy and Astrophysics, Berlin, v. 487, n. 3, p. 837-852, Sept. 2008.

39 KNEISKE, T. M.; DOLE, H. A lower-limit flux for the extragalactic background light. Astronomy and Astrophysics, Berlin, v. 515, p. A19, June 2010.

40 Jorgen Paul Rachen. Interaction Processes and Statistical Properties of the Propagation of Cosmic Rays in Photon Backgrounds. 1996. Tese (Doutorado em Física) - Universitat Bonn, Bonn, 1996.

41 SUPANITSKY, A.; SOUZA, V. D. An upper limit on the cosmic-ray luminosity of individual sources from gamma-ray observations. Journal of Cosmology and Astroparticle Physics, v. 2013, n. 12, p. 023-023, Dec. 2013.

42 ANJOS, R.; DE SOUZA, V.; SUPANITSKY, A. Upper limits on the total cosmic-ray luminosity of individual sources. Journal of Cosmology and Astroparticle Physics, v. 2014, n. 07, p. 049-049, July 2014.

43 HILLAS, A. M. The Origin of Ultra-High-Energy Cosmic Rays. Annual Review of Astronomy and Astrophysics, v. 22, n. 1, p. 425-444, Sept. 1984.

44 STANEV, T.; BIERMANN, P. L.; GAISSER, T. K. Cosmic rays. IV. The spectrum and chemical composition above 10' GeV. Astronomy and Astrophysics, Berlin, v. 274, p. 902, July 1993.

45 PEIXOTO, C. T.; DE SOUZA, V.; BIERMANN, P. L. Cosmic rays: the spectrum and chemical composition from $10^{10}$ to $10^{20} \mathrm{eV}$. Journal of Cosmology and Astroparticle Physics, v. 2015, n. 07, p. 042-042, July 2015.

46 Gopal-Krishna; BIERMANN, P. L.; SOUZA, V. D.; WIITA, P. J. Ultra-high-energy Cosmic Rays from Centaurus A: Jet Interaction with Gaseous Shells. The Astrophysical Journal Letters, v. 720, n. 2, p. L155, 2010. 
47 BIERMANN, P. L.; DE SOUZA, V. Centaurus A: The Extragalactic Source of Cosmic Rays with Energies above the Knee. The Astrophysical Journal, v. 746, n. 1, p. 72, 2012.

48 AHN, H. S. et al. DISCREPANT HARDENING OBSERVED IN COSMIC-RAY ELEMENTAL SPECTRA. The Astrophysical Journal, v. 714, n. 1, p. L89-L93, May 2010.

49 AHN, H. S. et al. ENERGY SPECTRA OF COSMIC-RAY NUCLEI AT HIGH ENERGIES. The Astrophysical Journal, v. 707, n. 1, p. 593-603, Dec. 2009.

50 S. Ting for the AMS Collaboration. The Alpha Magnetic Spectrometer on the International Space Station Highlight Talk. In: $33^{\text {th }}$ Int. Cosmic Ray Conf. Rio de Janeiro: , c2013.

51 ANTONI, T. et al. A non-parametric approach to infer the energy spectrum and the mass composition of cosmic rays. Astroparticle Physics, v. 16, n. 3, p. 245-263, Jan. 2002.

52 ANTONI, T. et al. KASCADE measurements of energy spectra for elemental groups of cosmic rays: Results and open problems. Astroparticle Physics, v. 24, n. 1-2, p. 1-25, Sept. 2005.

53 Marcel René Finger. Reconstruction of energy spectra for different mass groups of highenergy cosmic rays. 2011. Tese (Doutorado em Física) - Karlsruher Instituts für Technologie (KIT), 2011.

54 FUHRMANN, D.; ET AL., K. KASCADE-Grande measurements of energy spectra for elemental groups of cosmic rays. In: $32^{\text {rd }}$ Int. Cosmic Ray Conf. Beijing: , c2011.

55 The Pierre Auger Collaboration et al. Depth of maximum of air-shower profiles at the Pierre Auger Observatory. II. Composition implications. Physical Review D, v. 90, n. 12, Dec. 2014.

56 HORAN, D.; WEEKES, T. C. Extragalactic Sources of TeV Gamma Rays: A Summary. New Astronomy Reviews, v. 48, n. 5-6, p. 527-535, Apr. 2004. arXiv: astro-ph/0310391.

57 ABDO, A. A. et al. THE FIRST CATALOG OF ACTIVE GALACTIC NUCLEI DETECTED BY THE FERMI LARGE AREA TELESCOPE. The Astrophysical Journal, v. 715, n. 1, p. 429-457, May 2010.

58 ACKERMANN, $M$. et al. SEARCH FOR GAMMA-RAY EMISSION FROM X-RAYSELECTED SEYFERT GALAXIES WITH FERMI -LAT. The Astrophysical Journal, v. 747, n. 2, p. 104, Mar. 2012.

59 ALEKSlć, J. et al. DETECTION OF VERY HIGH ENERGY $\gamma$-RAY EMISSION FROM THE PERSEUS CLUSTER HEAD-TAIL GALAXY IC 310 BY THE MAGIC TELESCOPES. The Astrophysical Journal, v. 723, n. 2, p. L207-L212, Nov. 2010. 
60 GALANTE, N.; COLLABORATION, F. T. V. VERITAS Observations of Radio Galaxies. arXiv:0912.3850 [astro-ph], Dec. 2009. arXiv: 0912.3850.

61 AHARONIAN, F. et al. Upper limits from HESS active galactic nuclei observations in 2005-2007. Astronomy and Astrophysics, Berlin, v. 478, n. 2, p. 387-393, Feb. 2008.

62 SOMMERS, P. Capabilities of a giant hybrid air shower detector. Astroparticle Physics, v. 3, n. 4, p. 349-360, Aug. 1995.

63 WATSON, A. Ultra-high-energy cosmic rays: the experimental situation. Physics Reports, v. 333-334, p. 309-327, Aug. 2000.

64 The Pierre Auger Collaboration. The Pierre Auger Cosmic Ray Observatory. arXiv:1502.01323, Feb. 2015. arXiv: 1502.01323.

65 HILLAS, M. E. A. Calculations on the particle and energy-loss densities in extensive air showers at large axial distances. In: $11^{\mathrm{TH}}$ Int. Cosmic Ray Conf. Budapeste: , c1969. p. 533.

66 HERSIL, J.; ESCOBAR, I.; SCOTT, D.; CLARK, G.; OLBERT, S. Observations of Extensive Air Showers near the Maximum of Their Longitudinal Development. Physical Review Letters, v. 6, n. 1, p. 22-23, Jan. 1961.

67 The Pierre Auger Collaboration et al. The Pierre Auger Observatory: Contributions to the 33rd International Cosmic Ray Conference (ICRC 2013). arXiv:1307.5059, July 2013. arXiv: 1307.5059 .

68 The Pierre Auger Collaboration et al. LARGE SCALE DISTRIBUTION OF ULTRA HIGH ENERGY COSMIC RAYS DETECTED AT THE PIERRE AUGER OBSERVATORY WITH ZENITH ANGLES UP TO 80 DEGREES. The Astrophysical Journal, v. 802, n. 2, p. 111, Mar. 2015.

69 The Pierre Auger Collaboration et al. CONSTRAINTS ON THE ORIGIN OF COSMIC RAYS ABOVE $10^{18}$ eV FROM LARGE-SCALE ANISOTROPY SEARCHES IN DATA OF THE PIERRE AUGER OBSERVATORY. The Astrophysical Journal, v. 762, n. 1, p. L13, Jan. 2013.

70 BAUMGARTNER, W. H.; TUELLER, J.; MARKWARDT, C. B.; SKINNER, G. K.; BARTHELMY, S.; MUSHOTZKY, R. F.; EVANS, P. A.; GEHRELS, N. THE 70 MONTH SWIFT -BAT ALL-SKY HARD X-RAY SURVEY. The Astrophysical Journal Supplement Series, v. 207, n. 2, p. 19, Aug. 2013.

71 The Pierre Auger Collaboration et al. A SEARCH FOR POINT SOURCES OF EeV PHOTONS. The Astrophysical Journal, v. 789, n. 2, p. 160, July 2014.

72 The Pierre Auger Collaboration et al. Improved limit to the diffuse flux of ultrahigh energy neutrinos from the Pierre Auger Observatory. Physical Review D, v. 91, n. 9, May 2015. 
73 HöCKER, A.; KARTVELISHVILI, V. SVD approach to data unfolding. Nuclear Instruments and Methods in Physics Research Section A: Accelerators, Spectrometers, Detectors and Associated Equipment, v. 372, n. 3, p. 469-481, Apr. 1996.

74 D'AGOSTINI, G. A multidimensional unfolding method based on Bayes' theorem. Nuclear Instruments and Methods in Physics Research Section A: Accelerators, Spectrometers, Detectors and Associated Equipment, v. 362, n. 2-3, p. 487-498, 1995.

75 Hansen, P.C. Discrete Inverse Problems: Insight and Algorithms. SIAM, 3600 Market Street, Floor 6, Philadelphia, PA 19104: Society for Industrial and Applied Mathematics, 2010.

76 PIEROG, T.; ALEKSEEVA, M.; BERGMANN, T.; CHERNATKIN, V.; ENGEL, R.; HECK, D.; KALMYKOV, N.; MOYON, J.; OSTAPCHENKO, S.; THOUW, T.; WERNER, K. First results of fast one-dimensional hybrid simulation of EAS using CONEX. Nuclear Physics $B$ Proceedings Supplements, v. 151, n. 1, p. 159-162, Jan. 2006.

77 The Pierre Auger Collaboration et al. The fluorescence detector of the Pierre Auger Observatory. Nuclear Instruments and Methods in Physics Research Section A: Accelerators, Spectrometers, Detectors and Associated Equipment, v. 620, n. 2-3, p. 227-251, Aug. 2010.

78 LINSLEY, J.; WATSON, A. A. Validity of Scaling to $10^{20} \mathrm{eV}$ and High-Energy Cosmic-Ray Composition. Physical Review Letters, v. 46, n. 7, p. 459-463, Feb. 1981.

79 OLINTO, A. Ultra high energy cosmic rays: the theoretical challenge. Physics Reports, v. 333-334, p. 329-348, Aug. 2000.

80 GREISEN, K. End to the Cosmic-Ray Spectrum? Phys. Rev. Lett., v. 16, n. 17, p. 748-, Apr. 1966.

81 ZATSEPIN, G. T.; KUZ'MIN, V. A. ZhETF Pis'ma, v. 4, p. 114, 1966.

82 The Pierre Auger Collaboration et al. SEARCHES FOR LARGE-SCALE ANISOTROPY IN THE ARRIVAL DIRECTIONS OF COSMIC RAYS DETECTED ABOVE ENERGY OF $10^{19} \mathrm{eV}$ AT THE PIERRE AUGER OBSERVATORY AND THE TELESCOPE ARRAY. The Astrophysical Journal, v. 794, n. 2, p. 172, Oct. 2014.

83 The Telescope Array Collaboration et al. INDICATIONS OF INTERMEDIATE-SCALE ANISOTROPY OF COSMIC RAYS WITH ENERGY GREATER THAN $57 \mathrm{EeV}$ IN THE NORTHERN SKY MEASURED WITH THE SURFACE DETECTOR OF THE TELESCOPE ARRAY EXPERIMENT. The Astrophysical Journal, v. 790, n. 2, p. L21, Aug. 2014.

84 ABBASI, R.; BELLIDO, J.; BELZ, J.; DE SOUZA, V.; HANLON, W.; IKEDA, D.; LUNDQUIST, J. P.; SOKOLSKY, P.; STROMAN, T.; TAMEDA, Y.; TSUNESADA, Y.; UNGER, 
M.; YUSHKOV, A.; for the Pierre Auger Collaboration and the Telescope Array Collaboration. Report of the Working Group on the Composition of Ultra High Energy Cosmic Rays. arXiv:1503.07540, Mar. 2015.

85 KRONBERG, P. P. Extragalactic magnetic fields. Reports on Progress in Physics, v. 57, n. 4, p. 325-382, Apr. 1994.

86 KOTERA, K.; OLINTO, A. V. The Astrophysics of Ultrahigh-Energy Cosmic Rays. Annual Review of Astronomy and Astrophysics, v. 49, n. 1, p. 119-153, Sept. 2011.

87 KEIVANI, A.; FARRAR, G. R.; SUTHERLAND, M. Magnetic deflections of ultra-high energy cosmic rays from Centaurus A. Astroparticle Physics, v. 61, p. 47-55, Feb. 2015.

88 LEE, S.; OLINTO, A. V.; SIGL, G. Extragalactic Magnetic Field and the Highest Energy Cosmic Rays. The Astrophysical Journal, v. 455, n. 1, Dec. 1995.

89 LEMOINE, M.; SIGL, G.; OLINTO, A. V.; SCHRAMM, D. N. Ultra-high-Energy CosmicRay Sources and Large-Scale Magnetic Fields. The Astrophysical Journal, v. 486, n. 2, p. L115-L118, Sept. 1997.

90 Andrew Martin Taylor. The Propagation of Ultra High Energy Cosmic Rays. 2006. Tese (Doutorado em Física) - Universityof Oxford, Oxford, 2006.

91 BENNETT, C. L.; BANDAY, A. J.; GóRSKI, K. M.; HINSHAW, G.; JACKSON, P.; KEEGSTRA, P.; KOGUT, A.; SMOOT, G. F.; WILKINSON, D. T.; WRIGHT, E. L. FourYear COBE DMR Cosmic Microwave Background Observations: Maps and Basic Results. The Astrophysical Journal, v. 464, n. 1, p. L1-L4, June 1996.

92 HINSHAW, G. et al. Three-Year Wilkinson Microwave Anisotropy Probe ( WMAP ) Observations: Temperature Analysis. The Astrophysical Journal Supplement Series, v. 170, n. 2, p. 288-334, June 2007.

93 Planck Collaboration et al. Planck 2013 results. I. Overview of products and scientific results. Astronomy \& Astrophysics, v. 571, p. A1, Nov. 2014.

94 ESSEY, W.; KALASHEV, O. E.; KUSENKO, A.; BEACOM, J. F. Secondary Photons and Neutrinos from Cosmic Rays Produced by Distant Blazars. Physical Review Letters, v. 104, n. 14, Apr. 2010.

95 ESSEY, W.; KALASHEV, O.; KUSENKO, A.; BEACOM, J. F. Role of line-of-sight cosmic ray interactions in forming the spectra of distant blazars in $\mathrm{TeV}$ gamma rays and high-energy neutrinos. The Astrophysical Journal, v. 731, n. 1, p. 51, Apr. 2011. arXiv: 1011.6340.

96 ESSEY, W.; KUSENKO, A. A new interpretation of the gamma-ray observations of active galactic nuclei. Astroparticle Physics, v. 33, n. 2, p. 81-85, Mar. 2010. arXiv: 0905.1162. 
97 WILSON, A. S.; YOUNG, A. J.; SHOPBELL, P. L. Chandra X-Ray Observations of Pictor A: High-Energy Cosmic Rays in a Radio Galaxy. The Astrophysical Journal, v. 547, n. 2, p. 740-753, Feb. 2001.

98 BROWN, A. M.; ADAMS, J. Discovery of $\gamma$-ray emission from the broad-line radio galaxy Pictor A: Discovery of $\gamma$-ray emission from Pictor A. Monthly Notices of the Royal Astronomical Society, London, v. 421, n. 3, p. 2303-2309, Apr. 2012.

99 TINGAY, S. J.; LENC, E.; BRUNETTI, G.; BONDI, M. A HIGH RESOLUTION VIEW OF THE JET TERMINATION SHOCK IN A HOT SPOT OF THE NEARBY RADIO GALAXY PICTOR A: IMPLICATIONS FOR X-RAY MODELS OF RADIO GALAXY HOT SPOTS. The Astronomical Journal, v. 136, n. 6, p. 2473-2482, Dec. 2008.

100 PERLEY, R. A.; ROSER, H.-J.; MEISENHEIMER, K. The radio galaxy PictorA - a study with the VLA. Astronomy and Astrophysics, Berlin, v. 328, p. 12-32, Dec. 1997.

101 HILLAS, M. Cosmic Rays: Recent Progress and some Current Questions. arXiv, 2006. astro-ph/0607109.

102 LOVELACE, R. V. E. Dynamo model of double radio sources. Nature, v. 262, n. 5570, p. 649-652, Aug. 1976.

103 KAMPERT, K.-H.; ET AL. CRPropa 2.0 - a Public Framework for Propagating High Energy Nuclei, Secondary Gamma Rays and Neutrinos. Astopart. Phys., v. 42, p. 41, 2013.

104 The Pierre Auger Collaboration. Update on the measurement of the CR energy spectrum above $10^{18} \mathrm{eV}$ made using the Pierre Auger Observatory. In: PROCEEDINGS OF THE 32ND International Cosmic Ray Conference. Beijing: , c2011.

105 FERMI, E. On the Origin of the Cosmic Radiation. Phys. Rev., v. 75, n. 8, p. 1169, Apr. 1949.

106 BLANDFORD, R. D.; OSTRIKER, J. P. Particle acceleration by astrophysical shocks. The Astrophysical Journal Letters, v. 221, p. L29-L32, Apr. 1978.

107 KRYMSKII, G. F.; PETUKHOV, S. I.; TRANSKII, I. A.; MAMRUKOVA, V. P.; SHAFER, G. V. Cosmic-ray distribution in the vicinity of the front of a nonstationary shock wave. Geomagnetism and Aeronomy, v. 16, p. 25-29, Aug. 1976.

108 AXFORD, W. I.; LEER, E.; SKADRON, G. The acceleration of cosmic rays by shock waves. International Cosmic Ray Conference, v. 11, p. 132-137, 1977.

109 BELL, A. R. The acceleration of cosmic rays in shock fronts. II. Monthly Notices of the Royal Astronomical Society, London, v. 182, p. 443-455, Feb. 1978. 
110 BELL, A. R. The acceleration of cosmic rays in shock fronts. I. Monthly Notices of the Royal Astronomical Society, London, v. 182, p. 147-156, Jan. 1978.

111 HAYASHIDA, N. et al. Observation of a Very Energetic Cosmic Ray Well Beyond the Predicted $2.7 \mathrm{~K}$ Cutoff in the Primary Energy Spectrum. Physical Review Letters, v. 73, n. 26, p. 3491-3494, Dec. 1994.

112 BHATTACHARJEE, P.; SIGL, G. Origin and propagation of extremely high-energy cosmic rays. Physics Reports, v. 327, n. 3-4, p. 109-247, Apr. 2000.

113 SIGL, G.; LEMOINE, M.; BIERMANN, P. Ultra-high energy cosmic ray propagation in the local supercluster. Astroparticle Physics, v. 10, n. 2-3, p. 141-156, Mar. 1999.

114 BELL, A. R.; LUCEK, S. G. Cosmic ray acceleration to very high energy through the non-linear amplification by cosmic rays of the seed magnetic field. Monthly Notices of the Royal Astronomical Society, London, v. 321, n. 3, p. 433-438, Mar. 2001.

115 CAPRIOLI, D.; AMATO, E.; BLASI, P. Non-linear diffusive shock acceleration with free-escape boundary. Astroparticle Physics, v. 33, n. 5-6, p. 307-311, June 2010.

116 BYKOV, A. M.; OSIPOV, S. M.; ELLISON, D. C. Cosmic ray current driven turbulence in shocks with efficient particle acceleration: the oblique, long-wavelength mode instability: CR current driven turbulence in shocks. Monthly Notices of the Royal Astronomical Society, London, v. 410, n. 1, p. 39-52, Jan. 2011.

117 LAGAGE, P. O.; CESARSKY, C. J. The maximum energy of cosmic rays accelerated by supernova shocks. Astronomy and Astrophysics, Berlin, v. 125, p. 249-257, Sept. 1983.

118 BIERMANN, P. L.; BECKER, J. K.; DREYER, J.; MELI, A.; SEO, E.-S.; STANEV, T. THE ORIGIN OF COSMIC RAYS: EXPLOSIONS OF MASSIVE STARS WITH MAGNETIC WINDS AND THEIR SUPERNOVA MECHANISM. The Astrophysical Journal, v. 725, n. 1, p. 184-187, Dec. 2010.

119 BISNOVATYI-KOGAN, G. S.; MOISEENKO, S. G. Core-collapse supernovae: magnetorotational mechanism. Astronomical \& Astrophysical Transactions, v. 26, n. 1-3, p. 71-74, June 2007.

120 RACHEN, J. P.; BIERMANN, P. L. Extragalactic Ultra-High Energy Cosmic-Rays - Part One - Contribution from Hot Spots in Fr-Il Radio Galaxies. Astronomy and Astrophysics, Berlin, v. 272, p. 161, May 1993.

121 RACHEN, J. P.; STANEV, T.; BIERMANN, P. L. Extragalactic ultra-high energy cosmic rays. II. Comparison with experimental data. Astronomy and Astrophysics, Berlin, v. 273, p. 377, June 1993. 
122 BIERMANN, P. L.; BECKER, J. K.; CARAMETE, L.; CURUTIU, A.; ENGEL, R.; FALCKE, H.; GERGELY, L. A.; ISAR, P. G.; MARIS, I. C.; MELI, A.; KAMPERT, K.-H.; STANEV, T.; TASCAU, O.; ZIER, C. Active Galactic Nuclei: Sources for ultra high energy cosmic rays? Nuclear Physics B - Proceedings Supplements, v. 190, p. 61-78, May 2009. arXiv: 0811.1848.

123 BAADE, W.; MINKOWSKI, R. On the Indentification of Radio Sources. The Astrophysical Journal, v. 119, p. 215, Jan. 1954.

124 QUINN, P. J. On the formation and dynamics of shells around elliptical galaxies. The Astrophysical Journal, v. 279, p. 596, Apr. 1984.

125 OOSTERLOO, T. A.; MORGANTI, R. Anomalous HI kinematics in Centaurus A: Evidence for jet-induced star formation. Astronomy and Astrophysics, Berlin, v. 429, n. 2, p. 469-475, Jan. 2005.

126 KRAFT, R. P.; FORMAN, W. R.; HARDCASTLE, M. J.; BIRKINSHAW, M.; CROSTON, J. H.; JONES, C.; NULSEN, P. E. J.; WORRALL, D. M.; MURRAY, S. S. THE JET HEATED $X$-RAY FILAMENT IN THE CENTAURUS A NORTHERN MIDDLE RADIO LOBE. The Astrophysical Journal, v. 698, n. 2, p. 2036-2047, June 2009.

127 MALIN, D. F.; QUINN, P. J.; GRAHAM, J. A. Shell structure in NGC 5128. The Astrophysical Journal, v. 272, p. L5, Sept. 1983.

128 SCHIMINOVICH, D.; VAN GORKOM, J. H.; VAN DER HULST, J. M.; KASOW, S. Discovery of Neutral Hydrogen Associated with the Diffuse Shells of NGC 5128 (Centaurus A). The Astrophysical Journal, v. 423, p. L101, Mar. 1994.

129 CHARMANDARIS, V.; COMBES, F.; VAN DER HULST, J. M. First detection of molecular gas in the shells of CenA. Astronomy and Astrophysics, Berlin, v. 356, p. L1-L4, Apr. 2000.

130 Gopal-Krishna; SARIPALLI, L. Possible relationship between the radio and optical structures associated with the active galaxy Centaurus A. Astronomy and Astrophysics, Berlin, v. 141, p. 61-66, Dec. 1984.

131 Gopal-Krishna; WIITA, P. J. Galaxy shells and the structure of radio galaxies: Clues from Centaurus A (NGC 5128). New Astronomy, v. 15, n. 1, p. 96-101, Jan. 2010.

132 CHOI, E.; WIITA, P. J.; RYU, D. Hydrodynamic Interactions of Relativistic Extragalactic Jets with Dense Clouds. The Astrophysical Journal, v. 655, n. 2, p. 769-780, Feb. 2007.

133 WHYSONG, D.; ANTONUCCI, R. New insights on selected radio galaxy nuclei. New Astronomy Reviews, v. 47, n. 4-5, p. 219-223, Sept. 2003. 
134 The Fermi-LAT Collaboration et al. Fermi Gamma-Ray Imaging of a Radio Galaxy. Science, v. 328, n. 5979, p. 725-729, May 2010.

135 AHARONIAN, F. et al. DISCOVERY OF VERY HIGH ENERGY $\gamma$-RAY EMISSION FROM CENTAURUS A WITH H.E.S.S. The Astrophysical Journal, v. 695, n. 1, p. L40-L44, Apr. 2009.

136 ABBASI, R. et al. Monocular measurement of the spectrum of UHE cosmic rays by the FADC detector of the HiRes experiment. Astroparticle Physics, v. 23, n. 2, p. 157-174, Mar. 2005.

137 Pedro Abreu; et al. Muon Array with RPCs for Tagging Air showers (MARTA). In: . The Hague: , c2015.

138 KILDEA, J.; ATKINS, R.; BADRAN, H.; BLAYLOCK, G.; BOND, I.; BRADBURY, S.; BUCKLEY, J.; CARTERLEWIS, D.; CELIK, O.; CHOW, Y. The Whipple Observatory 10m gamma-ray telescope, 1997-2006. Astroparticle Physics, v. 28, n. 2, p. 182-195, Oct. 2007.

139 BERNLOHR, K. The optical system of the H.E.S.S. imaging atmospheric Cherenkov telescopes. Part I: layout and components of the system. Astroparticle Physics, v. 20, n. 2, p. 111-128, 2003.

$140 \mathrm{KAWACHI}, \mathrm{A}$. The optical reflector system for the CANGAROO-II imaging atmospheric Cherenkov telescope. Astroparticle Physics, v. 14, n. 4, p. 261-269, Jan. 2001.

141 ACCIARI, V. A. et al. Observation of Gamma-Ray Emission from the Galaxy M87 above $250 \mathrm{GeV}$ with VERITAS1. The Astrophysical Journal, v. 679, n. 1, p. 397-403, May 2008.

142 The MAGIC Collaboration. The MAGIC gamma-ray observatory. Nuclear Instruments and Methods in Physics Research Section A: Accelerators, Spectrometers, Detectors and Associated Equipment, v. 553, n. 1-2, p. 274-281, Nov. 2005.

143 The CTA Consortium. Design concepts for the Cherenkov Telescope Array CTA: an advanced facility for ground-based high-energy gamma-ray astronomy. Experimental Astronomy, v. 32, n. 3, p. 193-316, Nov. 2011. 